\title{
ESTIMADORES DE BAYES. APLICAÇÃO AO MODELO DE REGRESSÃO LINEAR SIMPLES
}

\section{NEWMAN RIBEIRO SIMŌES}

Orientador: Prof. Dr. Décio Barbin

Dissertação apresentada à Escola Superior de Agricultura "Luiz de Queiroz», da Universidade de São Paulo, para obtenção do título de Mestre em Estatística e Experimentação Agrorômica.

P I R A C I C A B A

Estado de São Paulo - Brasil 
ii.

A meu pai (in memoricon)

A minha mãe

A Beth, minha esposa,

Ao Ricardo, meu filho, pelo amor que nos une, DEDICO. 
Ao Professor Dr. Décio Barbin, pela orientação segura e valiosa prestada em todo Curso de Póș-Graduação.

A todos os meus amigos e colegas do Curso e Colégio Luiz de Queiroz (CLQ), pela profícua convivência e pelo estímulo no desenvolvimento deste trabalho.

Ao Professor Dr. Cássio Roberto de Melo Godoi, pelas crīticas, sugestões valiosas e pela elaboração do programa de compu tador.

Aos Professores do Departamento de Matemática e Esta tística da ESALQ, pela dedicação prestimosa na transmissão dos ensi namentos durante o Curso dẻ Pós-Graduação. Ao Professor Dr. Rodolfo Hoffmann, pelas sugestões críticas.

A Srta. Maria Izalina Ferreira Alves, pela amizade e pela admirável precisão na datilografia deste trabalho. Aos meus familiares, pela compreensão e o: incentivo constante. 
Pág.

RESUMO

vii

SUMMARY

ix

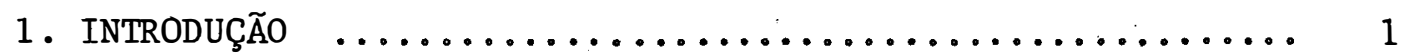

2. REVISÃO DE LITERATURA $\ldots \ldots \ldots \ldots \ldots \ldots \ldots \ldots \ldots \ldots \ldots \ldots$

2.1 - Elementos do Método de Bayes ................ 4

2.2 - Teoria da Informação e Informação "A Priori" ..... 9

2.3 - Estimadores de Bayes e Aplicação em Regressão Linear 13

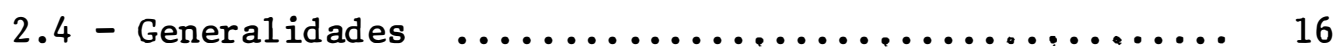

3. DESENVOLVIMENTO TEÓRICO $\ldots \ldots \ldots \ldots \ldots \ldots \ldots \ldots \ldots \ldots \ldots \ldots$

3.1 - Elementos do Método Bayesiano en Problemas de Estima

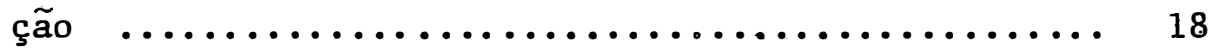

3.1 .1 - 0 teorema de Bayes $\ldots \ldots \ldots \ldots \ldots \ldots \ldots \ldots \ldots . . \ldots$

3.1 .2 - 0 método bayesiano na estimação de parâmetros 24

3.1.2.1 - A estimação como processo de tomada de decisão ................ 24

3.1.2.2 - Estimadores de Bayes ......... 27

3.1.2.3 - Especificação da Função Perda ... 29

3.2 - A Distribuição "A Priori" .................. 34

3.2 .1 - Generalidades $\ldots \ldots \ldots \ldots \ldots \ldots \ldots \ldots \ldots \ldots \ldots \ldots$

3.2.2 - o conceito de informação contida na amostra 37

3.2 .3 - Ausência de informação "a priori" ....... 47

3.2 .4 - Distribuições conjugadas $\ldots \ldots \ldots \ldots \ldots \ldots . \ldots 5$

3.3 - Aplicações em Modelo de Regressão Linear Simples .. 56 
Pāg.

3.3 .1 - 0 modelo e pressuposições

3.3.2 - Uso da f.d.p. indicando ausência de informa-

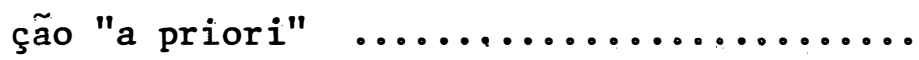

3.3.2.1 - F.D.P.s marginais "a posteriori" para os parâmetros $\ldots \ldots \ldots \ldots \ldots .61$

3.3.2.2 - Estimadores de Bayes para os parâmetros $\quad \ldots \ldots \ldots \ldots \ldots \ldots \ldots \ldots \ldots$

3.3.2.3 - Testes de hipóteses a respeito dos parâmetros ................ 65

3.3.2.4 - Intervalos de confiança para os pa râmetros ,.................. 72

3.3.2.5 - Intervalo de previsão ........ 74

3.3.3 - Uso da f.d.p. "a priori" informativa ..... 77

3.3.3.1 - F.D.P. "a priori" normal para os . coeficientes e variância residual conhecida ................. 77

3.3.3.2 - Uso da Distribuição Conjugada ... 84

4. COMPARAÇÃO DOS MẼTOdOS ATRAVES DE StMULAÇÃO DE DADOS $\ldots . . .91$

4.1 - Obtenção dos Dados $\ldots \ldots \ldots \ldots \ldots \ldots \ldots \ldots \ldots \ldots \ldots \ldots . \ldots \ldots$

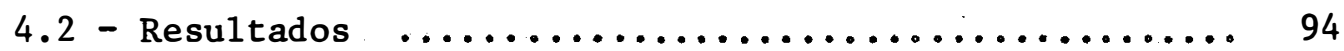

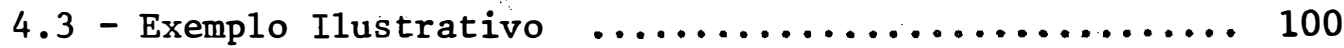

4.4 - Discussão dos Resultados ........................... 109

4.4 .1 - Estimativas dos parâmetros ............. 109 
vi.

Pāg.

4.4.2 - Estimativas das variâncias das estimativas dos parâmetros .................... 111

4.4 .3 - Intervalos de Confiança para os parâmetros.. 113

4.4.4 - Resultados gerais sobre a Análise de Regressão Simulada ........................ 113

4.5 - Sugestốes Para Outros Estudos .............. 115

5. CONCLUSÕES $\ldots \ldots \ldots \ldots \ldots \ldots \ldots \ldots \ldots \ldots \ldots \ldots \ldots \ldots \ldots \ldots$

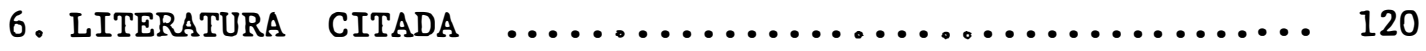

APENDICE I: PROGRAMA UTILIZADO NA STMULAÇÃO E ANÁLISE DOS DA

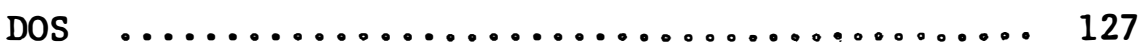

APENDICE II: REGRESSÕES COM VARIÂNCIAS DIFERENTES $\ldots \ldots \ldots \ldots . .131$ 
vii.

ESTIMADORES DE BAYES. APLICAÇÃO AO MODELO DE REGRESSÃO LINEAR SIMPLES

Autor: Newnan Ribeiro Simões

Orientador: Dr. Décio Barbin

Este estudo apresenta, através de revisão bibliogrāfica, os elementos do método de Bayes em problemas de estimação de parâmetros, assunto pouco difundido na lỉngua portuguesa.

O desenvolvimento teórico mostra, de um modo geral, como as informações anteriores a um experimento são incorporadas às informaçōes provindas de um experimento atual, através do Teorema de Bayes. São definidas Função Perda e Função Risco, mostrando como são obtidos os estimadores de Bayes quando se utiliza a função perda quadrática. Atenção particular é dada ao estudo da distribuição "a priori", reunindo algumas conclusōes de trabalhos sobre informação contida na amostra e sobre a distribuição "a priori" nas situações em que não se dispõe de informação prévia sobre os parâmetros. Dispondo de informações prévias, mostra-se como o método 
viii.

de Bayes pode utilizā-las, através das chamadas Distribuições Conjuga das.

Os conceitos gerais do método bayesiano são aplicados na estimação de parâmetros em Modelo de Regressão Linear Simples, encontrando-se, no caso de função perda quadrática, os estimadores de Bayes, as funções de densidade de probabilidade "a posteriori", os testes de hipóteses, intervalos de confiança para os parâmetros e in tervalo de previsão, nas situações em que a distribuição "a priori" indica ignorância sobre os parâmetros, e em que essa distribuição é informativa.

Experimentos são simulados para mostrar o comportamento das estimativas de Bayes em relação às estimativas de mínimos quadrados, particularmente no caso em que se dispõem de pequenas amostras atuais e informações prévias fidedignas, e na hipótese de que os coeficientes de regressão e as variâncias são os mesmos para as duas amostras. A robustez do método é verificada no caso em que esta hipótese é violada, modificando-se a variância dos dados da "amostra atua1" em relação aos dados da "amostra prévia". Um método aproximado para a análise desta ūltima situação é indicado. 


\title{
BAYES' ESTIMATORS, APPLICATION TO THE UNIVARIATE LINEAR REGRESS ION MODEL
}

\author{
Author: Newman Ribeiro Simões \\ Adviser: Dr. Décio Barbin
}

This study shows, through bibliographical revision, how the elements of Bayes' method are used in problems of parameter estimation, matter little diffused in portuguese language.

The theoretical development shows, by and large, how previous information can be incorporated to an actual experiment, through Bayes' theorem. Bayes' estimators based on quadratic loss are obtained with particular attention to prior distributions analysis, gathering some conclusions about information within sample and how to consider prior distributions in situations where no prior information do exist about parameters. Otherwise we show how to use Bayes' method, through Conjugated Distributions, when we do have prior information.

The general concepts of Bayesian Method are applied 
to the parameter estimation in the Univariate Normal Linear Regression Model, in the case of quadratic loss function. We look at Bayes' estimators, the posterior probability density functions, hypothesis tests, confidence intervals for the parameters and predictive inter val, in situations in which the prior distributions indicate ignorance about the parameters and when this distribution is informative.

Experiments are simulated to show the behaviour of Bayes' estimators compared to the least squares method, particularly in the case in which we have actual small sample size and creditable previous samples, and assuming regression coefficients and the variances being the same in the two samples. The robustness of the method is verified when this hypothesis is violated, changing the variance of the data of the actual sample compared to data of the past sample. An approximated method to deal with this last situation is indicated. 
1. INTRODUÇAOO

Ao delinear um experimento, estabelecer um modelo ma temático, estimar parâmetros, elaborar testes de hipóteses e analisar resultados, o estatístico utiliza teorias disponíveis, corroboradas pela evidência da aplicação prätica. Ā medida que estuda um fenô meno particular, o pesquisador incorpora novos conhecimentos sobre o mesmo e amplia sua visão sobre a relação que este fenômeno mantém com as situações mais gerais. E frequente, por exemplo, o estudioso sentir, por simples análise dos resultados, que o experimento não foi bem conduzido; ou que o modelo matemático foi inadequado. Entretanto, uma vez delineado o experimento, estabelecido o modelo matemático con soante com os fundamentos teóricos, as conclusões ficam restritas ao conjunto dos dados amostrais. Mas, em muitas aplicações, o pesquisador dispõe de informações fidedignas provindas de experiências ante- 
riores e que podem ser úteis para aumentar a precisão das estimativas, particularmente no caso de, no momento, só poder contar com pequenas amostras.

o método bayesiano considera, sob o enfoque da Teoria da Decisão, a possibilidade de incorporar na análise ambos os ti pos de informações: "anterior" ao experimento (por meio da expressão matemática da distribuição "a priori") e da evidência da experiência (através da função de verossimilhança da amostra), fornecendo a "dis tribuição 'a posteriori'".

Embora relativamente recente, o enfoque bayesiano da Estatística tem recebido contribuição crescente nos mais variados as pectos como a estimação de parâmetros, o da amostragem, o do estudo dos componentes de variância, testes de hipóteses, etc., o que se pode observar pelas publicações em livros e revistas especializadas.En tretanto, a revisão bibliográfica nos indica que com muito pouco podemos contar na literatura em português.

Como é um método que veio ampliar as perspectivas de alguns aspectos da anālise estatística, onde é de notória eficácia e, pelo fato de avançar rapidamente nas conquistas teóricas, é preciso abrir discussão a respeito e avaliar a sua utilização nas aplicações práticas, testar a sua eficiência e delimitar as suas restrições.

Cabe ressaltar que o método bayesiano não se contrapõe à Estatística Clássica, mas a amplia e the dá um aspecto particu lar. É lógico que para este avanço se processar foi necessário não 
só aparecerem novas fórmulas de trabalhoso manejo algébrico, como tam bém a reformulação de todo um conjunto de princípios e atitudes dian te dos problemas científicos da Estatística.

0 objetivo principal deste trabalho é discutir alguns aspectos gerais do processo de estimação bayesiano, reunindo diversos temas tratados em livros textos e artigos pouco divulgados na 1 in gua portuguesa. Sem a preocupação de apresentar coisas inéditas, pro curou-se aplicar o método de Bayes em uma situação particular, não muito explorada, que é o da estimação de parâmetros em regressão 1inear simples. Em virtude disto procurou-se acentuar a importância da revisão bibliogrä́fica, preparando o campo àqueles que tenham interes se em se aprofundar no assunto. 
4.

2. REVISÃO DE LITERATURA

\begin{abstract}
Este capítulo ē uma fonte indicadora de bibliografia para quem tiver necessidade de aprofundar os assuntos tratados nos capítulos seguintes. Não se trata, portanto, de leitura obrigatória para compreensāo do conteúdo deste trabalho.
\end{abstract}

\title{
2.1 - Elementos do Método de Bayes
}

Os fundamentos do método bayesiano, nos seus aspectos gerais, e os elementos necessārios para um estudo inicial,podem ser encontrados nos trabalhos a seguir.

BARNARD (1958) escreve nota bibliogräfica como intro dução à republicação do trabalho do Reverendo Thomas Bayes, em 1763, na qual desenvolve uma série de proposições a reṣpeito da adição e multiplicação de probabilidades, para concluir com o Teorema da Pró babilidade Inversa. 
JEFFREYS (1948), em livrro texto cọm primeira edição em 1939, sistematiza e amplia os conceitos relativos à inferência in dutiva e utiliza o teorema de Bayes para formalizar os mais diversos empregos da Matemática Estatística sob este novo enfoque, sendo considerado, ao lado de Savage e Wald, como precursor de métodos alternativos à Estatística Clássica.

WALD (1939) comenta restrições de Neyman ePearson a respeito de testes de hipóteses que, na determinação da região de re jeição do teste, depende do valor verdadeiro do parâmetro (para tornar mínima a probabilidade do erro tipo II). Introduz o conceito de "função ponderadora" ("weighted function"), que expressa a importância relativa do erro cometido ao se aceitar uma hipótese quando um valor particular do parâmetro é verdadeiro. A partir daí, e considerando a distribuição "a priori" para o parâmetro,formala as bases para o enfoque do teste de hipóteses através da teoria da decisão. Associado ao problema de escolher um "sistema admissível de região de aceitação", desenvolve o conceito de melhor estimador, demonstrando teoremas sobre suas propriedades. Mais tarde (1950), o mesmo autor completa este assunto em livro texto, concluindo que, com estes novos elementos, o melhor estimador para o verdadeiro valor desconheci do do parâmetro é o estimador de Bayes.

SAVAGE (1954), em livro texto, apresenta conceitos fundamentais para o desenvolvimento de tópicos como o de amostragem, estimação por pontos, testes de hipóteses e estimação por intervalo, 
através do enfoque da Teoria da Decisão. Apresenta algumas definições e discute as maneiras através das quais podem ser entendidos, sob o aspecto formal da matemática, conceitos como os de probabilidade sub jetiva, de utilidade e de função perda. 0 conteúdo do trabalho tem a maior importância pela maneira como é apresentado, solicitando do le tor uma postura diferente em relação aos problemas da Estatística pa ra a aceitação do método Minimax que o autor desenvolve,e que requer bases e fundamentos semelhantes aos do método de Bayes.

$$
\text { BARNARD (1954) apresenta alguns resultados obtidos }
$$
por WALD (1950) e LINDLEY (1953), de forma simplificada na sua demonstração, e amplia outros conceitos sobre funções de decisão estatística como generalização do lema fundamental de Neyman e Pearson. 0 autor demonstra um lema geométrico,a partir do qual o instrumento matemático utilizado na demonstração de um lema estatístico é simplí ficado.

PRATT, RAIFFA e SCHLAIFER (1964) desenvolvem os fundamentos da decisão em condições de incerteza, pela regra de Bayes, a partir de dois princípios que afirmam que quem decide pode encontrar sua escala de preferências sobre as consequências de sua decisão e julgamentos com relação a eventos não possíveis, baseados em experimento fictício, com ocorrências subjetivas e igualmente provāveis. Mostram que os dois princípios de comportamento consistente re querem que a escala de julgamento de quem decide deve obedecer aos axiomas de probabilidade, e que concordam com a usual definição de probabilidade condicional e com as regras de Bayes. 
LINDLEY (1965) assume o enfoque bayesiano como base para todo um livro texto dividido em duas partes. A primeira abordan do os conceitos usuais do estudo de probabilidade, dando ênfase espe cial à interpretação do Teorema de Bayes e suas aplicações naqueles conceitos. A segunda parte, dedicada à Inferência Estatística, desen volve e aprofunda a maneira bayesiana de análise, sempre comparando com os métodos usuais da verossimilhança e dos mínimos quadrados. Em bora tenha considerado praticamente só o caso de distribuição "a prio ri" indicando "ignorância" ou vago conhecimento, em nosso ponto de vista esta è uma obra indispensável à iniciação do estudo sob este enfoque da Inferência Estatística.

FERGUSON (1969) apresenta a Estatística Matemática, toda ela sob o ponto de vista da Teoria da Decisão introduzida por Wald. Um livro texto que aborda de maneira profunda os aspectos da Matemática envolvidos nos conceitos de probabilidade e estatística, sendo particularmente útil na abordagem formal dos conceitos de Utilidade e Probabilidade Subjetiva, usados no desenvolvimento dos processos Minimax e de Bayes. A leitura deste trabalho exige, para bom aproveitamento, o conhecimento avançado em probabilidade, análise no espaço euclidiano e conhecimento dos tópicos usuais da Estatística Matemātica.

Em ZACKS (1971) encontra-se uma abordagem mais profunda sobre os métodos de Inferência Estatística, incluindo capítulos especiais ao processo Minimax e ao mētodo de Bayes. 
MOOD e GRAYBILL (1972) apresentam, em 1ivro texto (Capítulo 8), as definições bāsicas para o entendimento e aplicação da Teoria da Decisão e, consequentemente, do método bayesiano de es timação. Função perda, função risco e risco esperado, são conceitos desenvolvidos e de fácil compreensão, sem, contudo, aprofundar suas ap1icações, citando o método bayesiano, sem assumi-lo no restante da abord agem .

De maneira semelhante o fazem FREEMAN (1963) e HOOG e CRAIG (1959), em seus livros textos. WONNACOTT e WONNACOTT (1976), também em livro texto sobre Econometria (Capítulo 10), apresentam uma introdução à Teoria da Decisão, com exemplos simples de aplicação do método bayesiano de estịmação e de testes de hipóteses.

SILVEY (1978), em monografia sobre Probabilidade Aplicada e Estatística, dedica o Capítulo 10 ao enfoque bayesiano,aprofundando alguns conceitos, como escolha da distribuição "a priori", distribuição "a priori" imprōpria e intervalo de confiança bayesiano. No capítulo seguinte apresenta uma introdução à Teoria da Decisão, abordando aspectos como os de função de decisão, função risco, problemas de decisão sequencial de Bayes. 0 "processo Minimax" de decisão, que se baseia nos mesmos conceitos iniciais do método de Bayes, è apresentado. 


\section{2 - Teoria da Informação e Informação "a priori"}

McMILLAN (1953) descreve os modelos matemáticos sob. os quais a teoria da comunicação é baseada e apresenta uma exposição com certos detalhes e critica parcial do tratamento de Shannon para cada problema. 0 trabalho parte da colocação de uma mensagem transmi tida estocasticamente e chega a uma descrição matemática que possibi lita o tratamento estatístico desta situação. Apresenta o conceito de entropia, com especificação de entropia marginal e entropia condicio nal média. Un teorema geral é desenvolvido para a teoria de processos estocásticos discretos.

LINDLEY (1956) introduz uma medida de informação for necida por um experimento, a partir do trabalho de Shannon e que,para delinear o experimento,exige o conhecimento "a priori" (expresso através da distribuição de probabilidade "a priori" sobre o espaço paramétrico). Estabelece, como aplicação desta medida de informaçá̃o, uma solução para alguns problemas de delineamento experimental quando o objetivo do experimento não é tomar decisões, mas adquirir conhecimento sobre o estado da natureza, objeto da pesquisa. 0 autor coloca, como condição básica para o estudo da informação, a aceitação da distribuição de probabilidades "a priori", e utiliza o teorema de Bayes para definir a quantidade de informação após a realização do experimento. Algumas definições e certas propriedades são for malizadas por teoremas que permitem comparar as informações provin- 
das de diversos experimentos. Exemplos são apresentados na situação em que duas consequências elementares são justificadas pelos conceitos apresentados, não envolvendo considerações de risco ou custo de experimentação, mas apenas a de ganhar e medir informação sobre o es tado da natureza. São elas: a) entre vários experimentos será esco1hido aquele em que a quantidade média de informação é maior; b) o estatístico termina o experimento quando tiver obtido informação suficiente.

Em 1957, LINDLEY discute métodos de amostragem em po. pulação binomial, com a finalidade de obter uma precisão pré-estabelecida ao se procurar encontrar a proporção desconhecida de uma caracterîstica estudada. Utiliza o conceito de Informação,devido a Shan non,e o conceito de medida de informação provinda de um experimento, apresentado pelo autor em 1956, a relação entre estes e o conceito de informação de Fisher para caracterizar esquemas amostrais. Estabe lece a equivalência assintótica da variância "a posteriori" do parâmetro e a variância de um estimador assintoticamente eficiente do mesmo.

RAIFFA e SCHLAIFFER (1961), em 1ivro texto,com abordagem ampla sobre aplicações da Teoria Estatística da Decisão, apresentam uma forma especial de distribuição "a priori conjugada" para representar a informação que se obtém através de experimentos anteriores. A distribuição "a priori conjugada" è admitida da mesma forma que aquela indicada pela verossimilhança da amostra e permite ob 
ter, através do método de Bayes, distribuição "a posteriori" da mesma forma. Todas as distribuições fundamentais de uso na Estatística são estudadas com este tipo de distribuição "a priori". Um aspecto interessante sobre como obter informação com finailidade de se tomar decisões através de amostragem é desenvolvido. RAIFFA (1977), em 1ivro texto, apresenta esta ü1tima abordagem sem considerar os aspectos matemáticos em profundidade, e fornecendo orientação no processo de tomada de decisão.

Propriedades de invariância de transformações bayesianas sobre o parâmetro, quando se usa a distribuição "a priori" pró posta por JEFFREYS (1948) para representar ausência de informação pré via (ou "ignorância") a respeito do parâmetro, são desenvolvidas por HARTIGAN (1964). O mesmo autor, em 1965, considerando estas condições, discute e propõe una forma de densidade de probabilidade "a priori" que minimiza assintoticamente o "viés" do estimador associado.

BARTHOLOMEW (1965) considera o problema de inferência sobre um único parâmetro, do qual depende a distribuição de uma variāvel aleatória, quando nenhuma informação "a priori" é disponíve1. Discute exemplos em que não é possível encontrar, pelo método bayesiano, propriedades requeridas pelos métodos clássicos. A magnitude da diferença entre afirmações probabilísticas pelos dois métodos é calculada discutindo a discrepância entre as duas teorias e su gerindo que é consequência da fixação do tamanho dos experimentos amostrais. 
WINKLER (1967) focaliza a determinação da distribuição "a priori" e alguns problemas da determinação que aparecem quando aquele que toma decisões deseja quantificar opiniões em condições de incerteza. Considera uma série de técniicas de determinação em proble mas estatísticos, juntamente com a instrução necessāria que estas téc nicas exigem para serem entendidas e aplicadas. Desenvolve um questionário que é usado em estudo no qual uma pessoa determina distribuições de probabilidades anteriormente à realizaçạo de um experimen to e os resultados indicam que, na maioria dos casos, é factível na determinação de distribuições de probabilidades subjetivas "a priori". Apresenta um questionārio revisado com indicações de seu uso potencial e sugere futuras pesquisas sobre o assunto estudado. - AKAIKE (1978) considera distribuição de probabilidade sobre o espaço paramétrico, ao obter estimativa da distribuição de uma futura observação, justificando assim o uso do procedimento baye siano na Inferência Estatística. Desenvolve um procedimento objetivo na avaliação da distribuição "a priori" indicando "ignorância" a res peito dos parâmetros e interpretada como distribuição imparcial. Utí liza o conceito de entropia na escolha da "função ganho" ao tomar uma densidade como estimativa da verdadeira densidade de probabilida de de uma distribuição. 


\section{3 - Estimadores de Bayes e Aplicação em Regressão Linear}

O método Bayesiano é utilizado por EVANS (1964) para encontrar novas formas gerais de estimadores da variância de distri buição normal e as condições sob as quais estas formas conduzem a es timadores, anteriormente proposto por GOODMAN, comparando diversas funções perda. Utiliza densidade "a priori" conjugada.

PRATT (1965) apresenta interpretações bayesianas sobre as afirmações padrões da inferência estatística. Estimação, estą tísticas suficientes, estimador imparcial, estimador consistente, re gião de confiança e nível de significância em testes de hipóteses, são conceitos que o autor apresenta sob o ponto de vista do método de Bayes.

BICKEL e BLACKWELL (1967) desenvolvem teoremas envo1 vendo estimadores de Bayes não tendenciosos e mostram que o risco de Bayes é nulo se a função perda é quadrática.

EFRON e MORRIS (1971) consideram o problema de estimação bayesiana da média da distribuição normal quando a distribuição "a priori" da própria média é normal. Mostram que o estimador usual de Bayes, nesta situação, apresenta alto risco se a verdadeira média é afastada da média da distribuição "a priori". Sugerem regras que não apresentam esta propriedade indesejävel.

TIAO e ZELLNER (1964) utilizam o enfoque bayesiano

para combinar a informação obtida pela amostra como conhecimento prẹ 
vio para se fazer inferências sobre os parâmetros do modelo de regressão em amostragem subsequente. Os autores consideram diversas si tuações com relação às variâncias, conforme as duas amostras possam ou não serem consideradas da mesma população. Generalizam as conclusões para o caso de vários conjuntos de experimentos serem conduzidos em sequência ou não, mas em condições completamente diferentes. Expressão de aproximação assintótica é deduzida para superar complicações de cálculos no caso de muitos experimentos, rincipalmente quando o número de parâmetros ê elevado. Os autores citam conclusões semelhantes obtidas por THEIL (1963) através de método não-bayesiano e apresentam exemplo de aplicação.

Em 1965, 2ELLNER e CHETTY revisama dedução da função de densidade de previsão do modelo de regressão mültipla, estabe lecendo teorema geral sobre previsão ótima por pontos e mostram como esta densidade de previsão pode ser empregada em anālise de problemas econométricos de investimento. De maneira semelhante, os autores desenvolvem a função de densidade de previsão em modelos de regressão multivariada e indicam seu uso em uma série de problemas.

LINDLEY e EL-SAYYAD (1968) desenvolvem o método baye siano aplicado à estimação de relação funcional linear; HARTIGAN (1969) propõe a aplicação do método em previsões lineares; LINDLEY e SMITH (1972) determinam os éstimadores de Bayes em regressão mū1tipla e SMITH (1973) amplia este estudo, observando com mais detalhes algumas propriedades gerais dos estimadores resultantes e compara, 
com exemplo, os resultados com aqueles obtidos pelo método dos mínimos quadrados.

ZELLNER (1971) desenvolve um trabalho criterioso,abor dando praticamente todos os aspectos da Inferência Estatística através do método de Bayes na Econometria. Após tratar dos princỉpios bayesianos em geral, a aplicação é apresentada em alguns tópicos da anālise de regressão, abordando os casos de erros autocorrelacionados, erros nas variáveis, modelos de séries temporais, modelos de re gressão multivariada, modelos de equações simultâneas e testes de hí póteses, sob o ponto de vista bayesiano.

$$
\text { ROTHEMBERG (1973) desenvolve interessante trabalho }
$$

sobre o uso de informação "a priori" para se obter estimação eficien te por pontos, com aplicações em anālise de regressão na Econometria e dá ênfase especial ao problema das equações simultâneas.Analisa co mo as informações, disponíveis antes de se realizar o experimento,po dem ser incorporadas no modelo pela teoria clássica da estimação, co mo é o caso da identificação dos parâmetros estruturais das equações simultâneas. Por outro lado, quando informações extras sobre os parâ metros são disponíveis, o método bayesiano é proposto como alternatị va eficiente de análise e exemplo numérico de aplicação é apresentado, comparando os resultados da estimação pelos dois processos. 
16.

\section{4 - Generalidades}

Alguns tōpicos mais específicos são encontrados em: AITCHISON (1964) que, comentando as dificuldades de formulação, desenvolvimento e interpretação da. região de tolerância pela teoria clássica, propõe a análise deste problema sob o ponto de vista bayesiano, indicando suas vantagens ao selecionar uma região ótima entre um conjunto de possíveis regiões de tolerância. THATCHER (1964), que analisa as relações entre previsão de Bayes e limite de confiança na situação em que, dado um número de sucessos em amostra aleatória, os limites de previsão podem ser determinados para o núme ro que será observado na amostra subsequente, por um caminho que não depende de suposições ou inferência sobre a proporção desconhecida na população. Duas diferentes distribuições "a priori" são utilizadas pa ra a regra de sucessão de Laplace e os resultados sugerem um tipo de "estratégia de previsão". o autor compara os valores numéricos das duas espécies de limites e explora a diferença conceitual entre ambos, mostrando que, para alguns casos, os resultados e interpretações são completamente diferentes.

PLACKETT (1966) explora as diferenças de opiniões e enfatiza as concordâncias entre a análise bayesiana, e a clássica.Dis cute alguns tópicos específicos, como a distribuição "a priori" e a obtenção de famílias de densidade conjugada.

LINDLEY (1972) revisou, de maneira geral, os mais va riados aspectos da estatística bayesiana através de monografia em que 
compara a eficiência deste enfoque com o da estatística clássica, usando argumentos puramente teóricos. E um importante trabalho que sin tetiza as conquistas do método bayesiano, bem como suas limitações atuais, comentando e orientando sobre os trabalhos publicados a respeito de cada item abordado. Uma bibliografia com indicação de 270 trabalhos è apresentada no final.

Finalmente citam-se algumas fontes que possibilitam a comparação do método bayesiano com os métodos clässjos, em questões importantes que se colocam na própria base de formulação concei tual de cada um dos processos. ZELLNER (1974) faz um comentário comparativo em problemas de Econometria, e TIAO e BOX (1974) comentam os estimadores de Bayes.

Alguns comentärios sobre assuntos específicos, mas que chegam a alcançar um aspecto de confronto entre bayesianos e não- bayesianos na formulação geral dos métodos, podem ser de interesse e encontrados nas discussões, por vārios autores, de alguns artigos já mencionados anteriormente, como em AITCHISON (1964), BORCH (1967), LINDLEY e SMITH (1972), PLACKETT (1966), PRATT (1965) e THATCHER (1964). 
18 .

3. DESENVOLVIMENTO TEÓRICO

3.1 - Elementos do Método Bayesiano em Problemas de Estimação

\subsection{1 - 0 Teorema de Bayes}

o Teorema de Bayes (algumas vezes chamado de Regra de Bayes ou Princípio da Probabilidade Inversa) foi estabelecido pelo Reverendo Thomas Bayes (170̣2-1761) no artigo "An Essay Toward Solving a Problem in the Doctrine of Chances" publicado postumamente (1763) e republicado em Biometrika (1958).

Basicamente o Teorema de Bayes é empregado na solução do seguinte problema: seja um evento aleatório E e sejam $A_{1}$; $A_{2}$; ...; $A_{n}$ eventos exaustivos e mutuamente exclusivos de um espaço amos tral S, tomados "a priori" como causas de E; com a realização de um experimento de resultado $E$, qual a probabilidade de que tenha sido causado por $\mathrm{A}_{i}$ ? 
19.

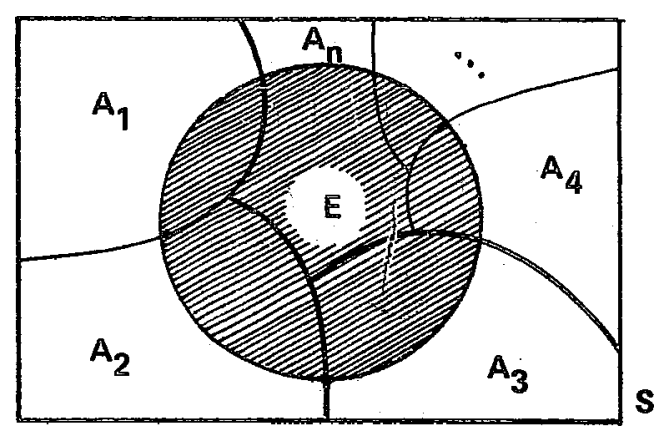

Figura 1 - Ilustração da demonstração do Teorema de Bayes.

P.S. Laplace, em Théorie Analytique des Probabilités, 1812, o enunciou explicitamente e,de maneira simples, é assim desenvolvido:

$$
\begin{aligned}
& E=S \cap E=\left(A_{1} \cup A_{2} \cup \ldots \cup A_{n}\right) \cap E \\
& E=\left(A_{1} \cap E\right) \cup\left(A_{2} \cap E\right) \cup \ldots \cup\left(A_{n} \cap E\right)
\end{aligned}
$$

onde os $\left(A_{i} \cap E\right)$ são mutuamente exclusivos (Figura 1 ).

$$
\text { Sejam: } P\left(A_{i}\right) \text { a probabilidade "a priori" de } A_{i} \text { (ou se }
$$
$j a$, na ignorância da realização de $E) ; P\left(E \mid A_{i}\right)$ a probabilidade condicional de E quando produzido pela causa $A_{i} ; P\left(A_{i} \mid E\right)$ a probabilidade "a posteriori" de $A_{i}$ (isto é, sua probabilidade dado que E foi verificado)

Ass im:

$$
\begin{aligned}
P(E)= & P\left(A_{1} \cap E\right)+P\left(A_{2} \cap E\right)+\ldots+P\left(A_{n} \cap E\right) \\
P(E)= & P\left(A_{1}\right) \cdot P\left(E \mid A_{1}\right)+P\left(A_{2}\right) \cdot P\left(E \mid A_{2}\right)+\ldots+ \\
& +P\left(A_{n}\right) \cdot P\left(E \mid A_{n}\right)
\end{aligned}
$$




$$
\begin{gathered}
E \text {, como } \\
P\left(A_{i} \mid E\right)=\frac{P\left(A_{i} \cap E\right)}{P(E)} \quad \text { e } \quad P\left(A_{i} \cap E\right)=P\left(A_{i}\right) \cdot P\left(E \mid A_{i}\right),
\end{gathered}
$$

tem-se:

$$
\begin{gathered}
P\left(A_{i} \mid E\right)=\frac{P\left(A_{i}\right) \cdot P\left(E \mid A_{i}\right)}{P\left(A_{1}\right) \cdot P\left(E \mid A_{1}\right)+P\left(A_{2}\right) \cdot P\left(E \mid A_{2}\right)+\ldots+P\left(A_{n}\right) \cdot P\left(E \mid A_{n}\right)} \\
\quad \therefore \quad P\left(A_{i} \mid E\right)=\frac{P\left(A_{i}\right) P\left(E \mid A_{i}\right)}{n} P\left(A_{j}\right) P\left(E \mid A_{j}\right)
\end{gathered}
$$

A utilização deste teorema como fundamento do método bayesiano na Estatística só foi possível com a evolução do conceito de probabilidade, baseado em fréquência relativa da ocorrência de eventos, para aquele que axiomatiza a teoria da medida de conjuntos (Kolmogoroff) e para outros conceitos mais atuais, como o da probabí lidade subjetiva. Particularmente,o conceito indutivo de probabilidade sistematizado por J.M. Keynes (1921) permitiu um avanço para que Jeffreys, com sua teoria da Inferência Indutiva, criasse as bases fundamentais para o emprego do teorema de Bayes em diversos aspectos da Inferência Estatística. Seu conceito fundamental é o de "grau de confiança" ("degree of belief") em uma proposição que satisfaz, certas regras de consistência e pode, em consequência destas regras, ser expressa formalmente por números ${ }^{(1)}$.

(1) Uma apresentação detalhada pode ser encontrada em SAVAGE (1954) pāgina 27 , sobre probabilidade subjetiva. 
Assim, passemos de eventos para proposições e de prọ babilidade de eventos para probabilidades de proposições que representam nosso "grau de confiança" na proposição e que é condicional ao nosso estado atual de informação sobre a mesma.

o Teorema de Bayes è usado para tornar operacional e quantitativo o processo de modificar probabilidade (representando "grau de confiança" em proposições) tendo em vista a incorporação de novas informações (geralmente como resultados de experimentos, considerações teóricas ou mesmo observação casual), como se pode obser var no esquema da Figura 2, apresentado por ZELLNER (1971).

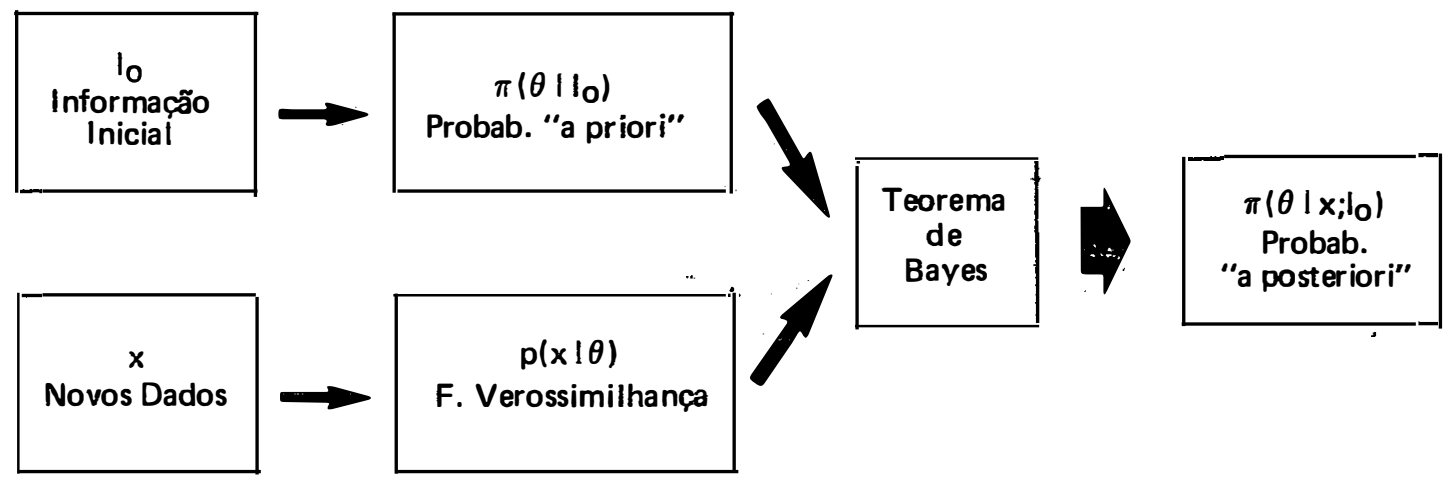

Figura 2 - 0 processo de revisão da probabilidade através de novos dados, pelo Teorema de Bayes.

Para utilizarmos (1.1) na estimação de parâmetros e descrever funções de densidade de probabilidade (f.d.p), consideremos os seguintes elementos: 
a) Espaço Paramétrico $(\Omega)$ : constituído por todos os valores pos síveis do parâmetro $(\theta)$,que é também chamado de "estado da natureza".

b) Espaço Amostral. (S): constituído por todos os valores que pode assumir a variável aleatória $x$, cuja distribuição de probabilí dade depende do estado da natureza $(\theta)$.

0 método bayesiano considera $\theta$ como variável aleatória que tem distribuição de probabilidade sobre $\Omega$. Como parâmetros são definidos como números fixos, esta consideração pode causar con trovérsias se não entendida pelo prisma de "grau de confiança" em uma proposição.Sendo um parâmetro, $\theta$ representa o estado real da na tureza e não significa que ocorra com certa probabilidade. Apenas que, tendo o seu valor verdadeiro desconhecido, pode ser tomado como variável aleatória pelos Estatísticos que decidem em situaçôes de incerteza.

\section{Assim representamos:}

a) $\pi\left(\theta \mid I_{0}\right):$ F.D.P. "a priori", que representa nosso "grau de confiança para os possíveis valores do parâmetro, baseado em nossa informação inicial $I_{0}$ (antes de conhecer a informação proveniente do experimento). (2)

b) $p(x \mid \theta)$ : função de verossimilihança da amostra.

c). $\pi(\theta \mid x ; I)$ : F.D.P. "a posteriori", que representa nosso "grau de confiança" para o parâmetro, após incorporar à nossa înformação inicial $\left(I_{0}\right)$ a informação proveniente do experimento.

(2) Para maiores detalhes sobre a informaçâo "a priori" e a f.d.p. "a priori", designamos um item especifico, adiante. 
Podemos estabelecer o teorema para variáveis aleatórias continuas, sendo que $\theta$ e $x$ podem representar valores singulares ou vetores aleatórios para os parâmetros e observações ${ }^{(3)}$.

Desta forma:

$$
\pi(\theta \mid x)=\frac{\pi(\theta) \cdot p(x \mid \theta)}{p(x)}
$$

onde $p(x)=\int_{\Omega} \pi(\theta) \cdot p(x \mid \theta) d \theta$

A expressão (1.2) pode ser escrita na forma:

$$
\pi(\theta \mid x) \propto \pi(\theta) \cdot p(x \mid \theta),
$$

onde $\propto$ indica proporcionalidade; $p(x)$ é a densidade marginal de $x$ e representa o recíproco da constante normalizadora da .f.d.p. de $(1.2)$, no sentido de que $\int_{\Omega} \pi(\theta \mid x) d \theta=1$.

A Figura 2 e a forma (1.2) do Teorema de Bayes móstram como nossa informação inicial sobre o estado da natureza, representada na f.d.p. "a priori", $\pi(\theta)$, é modificada pela evidência da experiência, representada pela informação provinda da amostra através da função de verossimilhança $p(x \mid \theta)$. Assim, ambas as informa ções são incorporadas em nosso estado atual de conhecimento sobre o fenômeno em estudo. Por outro lado, se dois conjuntos de dados $x_{1} e$ $x_{2}$ ocorrem em sequência, então a distribuição "a posteriori" para $x_{1}$

(3) Por facilidade de representação e por estar implícito no concei to, deixaremos de escrever I nas funções acima representadas.- 
pode ser "a priori" para $x_{2}$, indicando que, em condições gerais, a in formação provinda das amostras (na possibilidade de obtê-las em sequência) vai dominando a f.d.p. "a posteriori" que,por sua vez, tende rá ocorrer mais concentrada em torno do verdadeiro valor do parâmetro:

$$
\pi\left(\theta \mid \mathrm{x}_{1}\right) \propto \pi(\theta) \cdot \mathrm{p}\left(\mathrm{x}_{1} \mid \theta\right)
$$

que, usado como conhecimento "a priori" para um novo conjunto de da dos $x_{2}$, gerados independentemente de $x_{1}$, obtemos:

$$
\begin{aligned}
& \pi\left(\theta \mid x_{1} ; x_{2}\right) \propto \pi\left(\theta \mid x_{1}\right) \cdot p\left(x_{2} \mid \theta\right) \quad \text { ou } \\
& \pi\left(\theta \mid x_{1} ; x_{2}\right) \propto \pi(\theta) \cdot p\left(x_{1} \mid \theta\right) \cdot p\left(x_{2} \mid \theta\right) \quad
\end{aligned}
$$

Vê-se que (1.4) mostra que a f.d.p. para $\theta \bar{e}$ a mesma quando usamos o processo sequencial de $\pi(\theta)$ para $\pi\left(\theta \mid x_{1}\right)$ e então pa ra $\pi\left(\theta \mid x_{1}, x_{2}\right)$ ou quando usamos a função de verossimilhança para com binar amostras, através de $\mathrm{p}\left(\mathrm{x}_{1}, \mathrm{x}_{2} \mid \theta\right)$ em conjunção com $\pi(\theta)$.

\subsection{2 - 0 Método Bayesiano na estimação de parāmetros}

\subsubsection{1 - A estimação como processo de tomada de decisão}

A anālise estatística, sob o ponto de vista da decisão, tem sido desenvolvida, além do método bayesiano, pelo chamado Processo Minimax ${ }^{(4)}$. Não é intenção deste trabalho confrontā-los en tre si, mas apenas apresentar o enfoque bayesiano como um método al

(4) Sobre o Processo Minimax, dentro da bibliografia citada no final, pode-se encontrar detalhes nos trabalhos de SAVAGE (1954), FERGUSON (1969) e ZACKS (1971). 
ternativo ao tratamento clássico em certa classe de problemas.

A teoria estatística da decisão é concernente à anā1ise de experimentos aleatórios, com o propósito de tomar a melhor decisão sob condições de incerteza. De um modo geral, todos os aspectos da Estatística podem ser tratados sob este prisma e, particularmente, a teoria da estimação, os testes de hipóteses e interva los de confiança fazem parte de uma extensa lista bibliográfica de trabalhos teóricos e práticos da Estatística. Os conceitos fundamen tais e grande parte de sua teoria são devidos a WALD (1939 e 1950) e LEHMANN (1950). Nas palavras do primeiro (pág. 2): "Problemas de decisão estatística ocorrem quando estamos. face a um conjunto de de cisões alternativas, uma das quais deve ser tomada, e o grau de preferência pelas vārias possîveis decisões depende de distribuições desconhecidas de alguma variável aleatória".

Os efeitos da decisão tomada nos problemas estatísti cos dependem, assim, do desconhecido estado da natureza, representa do pelo vetor paramétrico (ou um parâmetro singular) $\theta$. Se para um problema especifico podemos definir um conjunto $A=\left\{a_{i}\right\}$ de todas as ações possiveis que se podem adotar, podemos assumir que todos os pa res $(a ; \theta)$ podem ser ordenados em preferência pela chamada Função Per da, $\underline{\ell}$, tal que $\ell(a ; \theta) \geqslant 0$ e que reflete a perda ao tomar a ação a quando o verdadeiro estado da natureza é $\theta$. Assim, $\ell\left(a_{1} ; \theta_{1}\right)<\ell\left(a_{2} ; \theta_{2}\right)$ é equivalente a afirmar que $\left(a_{1} ; \theta_{1}\right)$ é preferível a a $\left(a_{2} ; \theta_{2}\right)$. Se $\theta$ fosse conhecido, a melhor decisão seria selecionar a ação que minimi zasse $\ell(a ; \theta)$. Entretanto, $\theta$ não é conhecido e o problema de decisão ocorre. 
26.

Para se ter uma estrutura matemática do processo, ad mitimos que aquele que decide possa fundamentar sua decisão na observação do valor de uma variável aleatória, ou vetor, X, cuja distribuição também depende do verdadeiro estado da natureza. Com base na informação do experimento, quem decide escolhe a ação $a=d(x) \in A$

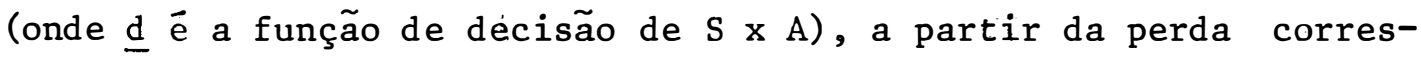
pondente $\ell(a ; \theta)=\ell\left[d(x) ; \theta^{-}\right]$.

Ainda que as funções $\underline{\ell}$ e $\underline{d}$ estejam determinadas, na prática a perda depende das observações particulares $x_{i}$, obtidas na amostra aleatória. Desta forma, a perda é, ela mesma, uma variável aleatooria e, para uma certa função de Decisão d e uma função Perda $\underline{\ell}$, definiremos a Função Risco ${ }^{(5)}-\mathrm{R}(\mathrm{d} ; \theta)$, como sendo o valor esperado da função Perda:

$$
\begin{aligned}
& \mathrm{R}(\mathrm{d} ; \theta)=\mathrm{E}[\ell(\mathrm{a} ; \theta)]=\mathrm{E}\{l[\mathrm{~d}(\mathrm{x}) ; \theta]\} \\
& \mathrm{R}(\mathrm{d} ; \theta)=\int_{S} \ell[\mathrm{d}(\mathrm{x}) ; \theta] \mathrm{P}(\mathrm{x} \mid \theta) \mathrm{dx}
\end{aligned}
$$

onde $p(x \mid \theta)$ é a f.d.p. de $x_{n}$ para dado $\theta$ e a integração é feita sobre o conjunto $S$ de possiveis valores amostrais $x_{n}$.

Como a função Risco depende da função de Decisão d escolhida para o problema, da função Perda $\underline{\ell}$ e do parâmetro desconhecido, admitimos como boa decisão aquela que torna mínimo o Risco para cada valor do parâmetro $\theta$.

A decisão será ötima $\left(d^{*}\right)$ se existir uma função de decisão que tenha o Risco uniformemente mínimo, ou seja:

(5) Um estudo detalhado sobre a Teoria do Risco pode ser encontrada em BORCH (1967), com discussão por vārios autores. 


$$
\mathrm{r}[-\mathrm{d} *(\mathrm{x}) ; \theta] \leq \mathrm{r}[\mathrm{d}(\mathrm{x}) ; \theta]
$$

para todo $\theta \in \Omega$ e para toda função d. Em geral tal função não existe e outros critérios serão necessários, como se faz na estimação usual que geralmente se restringe à adoção de estimadores não tendenciosos e de variância mínima.

Para o estatístico, nos problemas de estimação por

pontos, as funções de decisões são os estimadores e a decisão (ou ação) é a estimativa $\hat{\theta}$ do parâmetro.

\subsubsection{2 - Estimadores de Bayes}

Seja $x=\left(x_{1} ; x_{2} ; \ldots ; x_{n}\right)$ uma amostra aleatória de tamanho $\underline{\mathrm{n}}$ com densidade $\mathrm{p}(\mathrm{x} \mid \theta)$. Como vimos anteriormente, $\theta$ é cons $\underline{i}$ derado como variável aleatória de f.d.p. $\pi(\theta)$ e que, ao variar, determina diferentes densidades e a amostra aleatória foi tomada de uma destas densidades. Queremos estimar o valor particular de $\theta$ que determina tal densidade.

Os elementos necessārios serão, no método bayesiano:

a) $d(x)=d\left(x_{1}, \ldots, x_{n}\right)=\hat{\theta}$;

b) Função Perda: $\ell(\hat{\theta}, \theta)$;

c) Função Risco (ou simplesmente Risco): $R(d ; \theta)=E[\ell(\hat{\theta} ; \theta)]$.

Uma vez que $\theta$ é variável aleatória, determinaremos o valor $\theta *$ de $\theta$ que torna mínimo o Risco esperado: 
28.

$$
\theta *=\min _{\hat{\theta}} E[\ell(\bar{\theta} ; \theta)]=\min _{\hat{\theta}} \int_{\Omega} R(\mathrm{~d} ; \theta) \pi(\theta) \mathrm{d} \theta
$$

Por (1.5) podemos escrever:

$$
\theta^{*}=\min _{\hat{\theta}} \int_{\Omega}\left\{\int_{\mathrm{S}} \ell(\hat{\theta} ; \theta) \mathrm{p}(\mathrm{x} \mid \theta) \mathrm{d} \mathrm{x}\right\} \pi(\theta) \mathrm{d} \theta
$$

Considerando que sejam satisfeitas as condições para mudança na ordem de integração (em geral estas condições são satisfeitas), podemos escrever (1.7) na forma:

$$
\begin{aligned}
& \theta^{*}=\min _{\hat{\theta}} \int_{S}\left\{\int_{\Omega} \ell(\hat{\theta} ; \theta) \mathrm{p}(\mathrm{x} \mid \theta) \pi(\theta) \mathrm{d} \theta\right\} \mathrm{dx} \\
& \text { Mas, por }(1.2), \mathrm{p}(\mathrm{x} \mid \theta) \pi(\theta)=\mathrm{p}(\mathrm{x}) \pi(\theta \mid \mathrm{x}), \text { e a ex- }
\end{aligned}
$$

pressão de $\theta *$ fica:

$$
\theta *=\min _{\hat{\theta}} \int_{S}\left\{\int_{\Omega} \ell(\hat{\theta} ; \theta) \pi(\theta \mid x) d \theta\right\} p(x) d x
$$

Como $\mathrm{p}(\mathrm{x})$ é não negativa, podemos 'minimiżar oRisco esperado, minimizando a integral entre chaves de (1.8), para cada $x_{i}$ fixo. Em outras palavras, escolhemos $d^{*}(x)=\theta^{*}$ para cada $x_{i}$ fixo, co mo sendo a decisão que minimiza a Perda Esperada "a posteriori", chamada de Risco "a posteriori": $\overline{\mathrm{R}}(\mathrm{d} ; \theta)$.

Logo:

$$
\begin{aligned}
& \theta^{*}=\min _{\hat{\theta}} \int_{\Omega} \ell(\hat{\theta} ; \theta) \pi(\theta \mid \mathrm{x}) \mathrm{d} \theta \\
& \theta^{*}=\min _{\hat{\theta}}[\overline{\mathrm{R}}(\mathrm{d} ; \theta)]
\end{aligned}
$$


DEFINIÇÃO: Chama-se Estimador de Bayes para o parâmetro $\theta$, o valor de $\hat{\theta}$, em função da variāvel aleatooria $x$, que torna mỉnimo o Risco "a posteriori".

\subsubsection{3 - Especificação da função Perda}

Em problemas de estimação pouco se tem tratado sobre a função Perda ("loss function") - $\ell[\mathrm{d}(\mathrm{x}) ; \theta]$, sendo mais frequente nas discussões da teoria da decisão que, embora dependendo da estimação, considera outros aspectos mais específicos. E tambēm, pelo fato de que a interpretação da função Perda, não sendo muito clara, fez com que muitos autores desenvolvessem a análise bayesiana em ter mos da função Utilidade $-U(d ; \theta)$ de $A \times \Omega$ e que descreve o valor da decisão d quando o verdadeiro valor do parâmetro é $\theta$. Așsim, o problema é resolvido pela maximização da utilidade esperada "a posteriori":

$$
\overline{\mathrm{U}}(\mathrm{d} ; \theta)=\int_{\Omega} \mathrm{U}(\mathrm{d} ; \theta) \mathrm{p}(\theta \mid \mathrm{x}) \mathrm{d} \theta \quad \text {. }
$$

Uma discussão crítica sobre Utilidade pode ser encon trada em SAVAGE (1954) e, de maneira muito clara, em RAIFFA e SCHLAIFER (1961) que, numa linguagem matemática forma1, apresentam os diversos aspectos aplicados à Teoria da Decisão. PRATT (1964) mostra como a Utilidade pode ser descrita de maneira completa e satisfatória quando as consequências são inteiramente monetārias. Discute co mo podem ser medidas as implicações na forma de utilidade do montan 
te monetärio (z):

$$
u(z)=1-e^{-a z}-(1-w) e^{-b z},
$$

$a ; b>0$ e $0 \leq w \leq 1$

Convēm salientar que a existência e o preciso entendimento, bem como a mais precisa formalização na Teoria da Decisão, não invalidam e nem contrariam o uso da função Perda. A questão se coloca mais no âmbito da interpretação e utilização conveniente des tes dois conceitos.

A estimação por pontos, sob vārias funções Perda,foi discutida por BRITNEY e WINKLER (1968): EVANS (1964) considerou a estimação da variância da distribuição normal, levando em conta vários tipos de função Perda; EL-SAYYAD (1967), da mesma maneira, estudou a estimação em distribuição exponencial; ANTHEMAN (1965) abor da o problema da escolha do tamanho da amostra simples, levando em consideração a função Perda e dividindo a Perda em perda final e cus tos amostrais; VARIAN (1964) mostra uma situação prática (em estudo econômico de determinação de preço), onde a função perda quadrática é inapropriada por assinalar a mesma perda,tanto ao subestimar como ao superestimar o preço, propondo então uma função chamada "1inex" (1inear-exponencia1), da forma:

$$
L\left(y_{e} ; y_{a}\right)=b \cdot \exp \left[a\left(y_{e}-y_{a}\right)\right]-c\left(y_{e}-y_{a}\right)-b
$$

que, aplicada em modelo de regressão linear, mostrando ser uma expressão que bem se adapta ao modelo e à distribuição "a priori" nor 
mal-conjugada.

Em muitos problemas de decisão, entre os quais se in clui o de estimação de parâmetros,é muito difundido o uso da função Perda da forma:

$$
\ell(\hat{\theta} ; \theta)=c(\theta) \cdot(\hat{\theta}-\theta)^{2},
$$

onde $c(\theta)>0$ para todos os valores de $\theta$ e $\hat{\theta}=d(x)$.

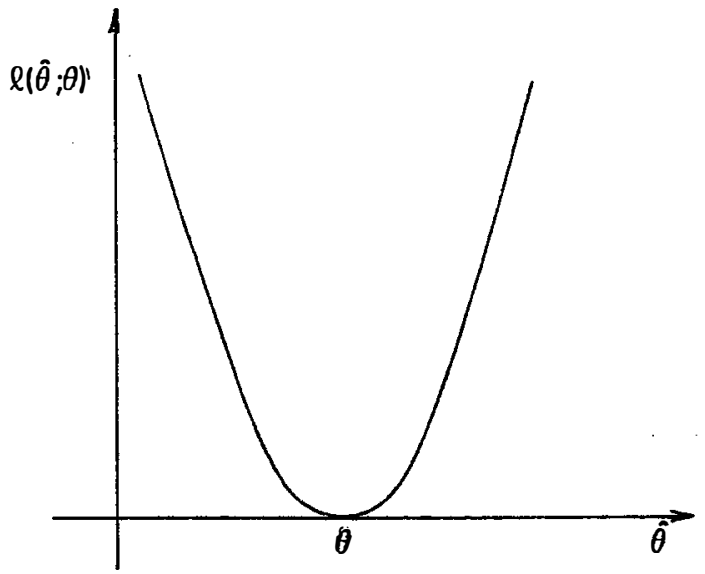

Figura 3 - Ilustração da função perda quadrática.

Neste caso a perda é maior quanto mais distante esti ver $\hat{\theta}$ do verdadeiro valor do parânetro e sua expressão, além de satisfazer a condição de definição de que $\ell(\hat{\theta} ; \theta) \geqslant 0$, mostra que a perda é nula se $\hat{\theta}$ representa o verdadeiro valor do parâmetro. Uma outra característica favorável ao seu uso é que é facilmente diferenciāvel nos problemas de minimização e mantém simples a estrutura algébrica da expressão da distribuição "a posteriori".

Uma dificuldade que aparece é como encontrar $c(\theta)$ que torne a perda realística, mas normalmente estamos interessados na 
comparação relativa de duas funções de decisão, no que c( $\theta)$ desempe nha papel secundārio.

Usando esta função Perda, o Risco será:

$$
R(d ; \theta)=E\left[c(\theta) \cdot(\hat{\theta}-\theta)^{2}\right]=c(\theta) \cdot E\left[(\hat{\theta}-\theta)^{2}\right]
$$

e, sendo $\hat{\theta}_{1}=d_{1}(x)$ e $\hat{\theta}_{2}=d_{2}(x)$ ta1 que $R\left(d_{1} ; \theta\right)>R\left(d_{2}>0\right)$, teremos :

$$
\begin{gathered}
\mathrm{E}\left[\ell\left(\hat{\theta}_{1} ; \theta\right)\right]>\mathrm{E}\left[\ell\left(\hat{\theta}_{2} ; \theta\right)\right] \\
\mathrm{c}(\theta) \cdot \mathrm{E}\left[\left(\hat{\theta}_{1}-\theta\right)^{2}\right]>c(\theta) \cdot \mathrm{E}\left[\left(\hat{\theta}_{2}-\theta\right)^{2}\right]
\end{gathered}
$$

e como $c(\theta)>0$,

$$
E\left[\left(\hat{\theta}_{1}-\theta\right)^{2}\right]>E\left[\left(\hat{\theta}_{2}-\theta\right)\right] .
$$

Esta desigualdade mostra que a comparação de Riscos para dois estimadores independe de $c(\theta)$.

Consideremos $\theta$ o vetor aleatōrio de parâmetros e M uma conhecida matriz simétrica definida positiva. Podemos definir a Função Perda Quadrática "a posteriori"

$$
\ell(\hat{\theta} ; \theta)=(\theta-\hat{\theta})^{\prime} . \mathbf{M} \cdot(\theta-\hat{\theta})
$$

e o Risco "a posteriori" será desenvolvido na forma:

$$
\begin{aligned}
& \overline{\mathrm{R}}(\mathrm{d} ; \theta)=\mathrm{E}[\ell(\hat{\theta} ; \theta)]=\mathrm{E}\left[(\theta-\hat{\theta})^{\prime} \cdot \mathrm{H}_{\cdot}(\theta-\hat{\theta})\right] \\
& =\mathrm{E}\{[(\theta-\mathrm{E} \theta)-(\hat{\theta}-\mathrm{E} \theta)] \cdot \cdot \mathrm{M} \cdot[(\theta-\mathrm{E} \theta)-(\hat{\theta}-\mathrm{E} \theta)]\} \\
& =E\left\{\begin{aligned}
(\theta-E \theta)^{\prime} \cdot M \cdot(\theta-E \theta) & -(\theta-E \theta)^{\prime} \cdot M \cdot(\hat{\theta}-E \theta)-(\hat{\theta}-E \theta)^{\prime} \cdot M \cdot(\theta-E \theta)+ \\
& +(\hat{\theta}-E \theta)^{\prime} \cdot M \cdot(\hat{\theta}-E \theta)
\end{aligned}\right\}
\end{aligned}
$$


33.

Como $\mathrm{E}(\theta-\mathrm{E} \theta)=0$, teremos:

$\overline{\mathrm{R}}(\mathrm{d} ; \theta)=\mathrm{E}\left[(\theta-\mathrm{E} \theta)^{\prime} \mathrm{M}(\theta-\mathrm{E} \theta)\right]+(\hat{\theta}-\mathrm{E} \theta)^{\prime} \mathrm{M}(\bar{\theta}-\mathrm{E} \theta)$

0 estimador de Bayes, de acordo com (1.9), é o valor de $\widehat{\theta}$ que minimiza o Risco "a posteriori". A primeira parcela do segundo membro de (1.10) não envolve $\hat{\theta}$ e, como Mé definida e positiva, a segunda parcela será minimizada se tomarmos

$$
\hat{\theta}=\mathrm{E} \theta \quad,
$$

ou seja,

$$
\theta *=\min _{\widehat{\theta}}[\bar{R}(\mathrm{~d} ; \theta)]=E(\theta) .
$$

Concluímos assim uma importante regra que será utilí zada nos problemas de estimação deste trabalho: quando usamos a fun ção Perda Quadrática, o estimador de Bayes é a média,E( $\theta)$, da distr $\underline{i}$ buição "a posteriori".

De maneira semelhante, demonstra-se que se a função Perda for $\ell(d ; \theta)=|\theta-\hat{\theta}|$ e a f.d.p. "a posteriori" é contínua e pró pria com $a \leq \theta \leq b$ (a e $\underline{b}$ conhecidos) o estimador ótimo por pontos é a Mediana da f.d.p. "a posteriori". 


\section{2 - A Distribuição "A Priori"}

\subsection{1 - Generalidades}

Como vimos em 3.1 , as afirmações probabilísticas a respeito do parâmetro $\theta$ (singular ou vetor de parâmetros) que repre senta o verdadeiro estado da natureza são feitas sob condições de incerteza e, assim, tomado no método bayesiano como variável aleatō ria. Daremos uma atenção especial a este ítem, que nos parece funda mental na caracterização do método adotado.

Sob o enfoque da Teoria da Decisão, o método bayesia no, particularmente na estimação de parâmetros, incorpora na análise ambas as informações: "anterior" ao experimento, através da expressão matemática da f.d.p. "a priori",e a evidência da experiência através da função de verossimilhança da amostra, fornecendo a distribuição "a posteriori".

De uma maneira geral, quem decide tem para $s j_{\text {i do }} \underline{i}$ pos de problemas:

19) tomar a melhor decisão com, base nos resultados de um experimento;

29) delinear o melhor experimento, de modo a colher informações nas quais a decisão serā baseada.

Parece-nos claro que, em ambas as situações, quem decide possui alguma informação "a priori", chegando, em caso extremo, a ter que tomar decisões sem oportunidade de delinear experimentos, 
baseando-se em conhecimentos resultantes de dados anteriores e que não estão sob o seu controle. Neste caso a solução de Bayes minimiza o "risco 'a priori""

$$
r^{*}(d)=\int_{\Omega} \int_{S} \ell(d ; \theta) \cdot f(x ; \theta) \cdot \pi(\theta) d x d \theta=\int_{\Omega} r(d ; \theta) \cdot \pi(\theta) d \theta,
$$

onde $\pi(\theta)$ é a f.d.p. "a priori" sobre o espaço paramétrico.

Pelos processos clássicos de estimação (mínimos quadrados e método da máxima verossimilhança), a informação "a priori" è caracterizada pelo conhecimento da formá da função de densidade e do espaço paramétrico,sendo a estimação avaliada com base na distribuição amostral, usando comumente o estimador de variância mínima na classe dos estimadores imparciais. 0 método de Bayes dispensa a imparcialidade, mas inclui a informação "a priori" sobre os parâmetros.

Quanto à natureza da informação "a priori", podemos ob tề-la através de dados passados, gerados por experimentos cientificamente conduzidos e que são disponỉveis para análises futuras. Em outras situações a informação "a priori" è resultado de observação casual, considerações teóricas e não provenientes de um conjunto par ticular de dados.Em qualquer situação,ou mesmo se ambos os tipos de informação são disponíveis, è necessārio avaliar a sua importância, no sentido de aumentar a precisão da estimativa dos parâmetros,particularmente em pequenas amostras. Se a perda em ignorar informação extra sobre os parâmetros é pequena, não há necessidade de desenvo $\underline{1}$ ver métodos algébricos complicados para incorporā-1a. Caso con- 
trārio, é valioso obter e usar tal informação em nosso trabalho estatístico.
Uma vez aceito que "graus de confiança" sobre os pa- râmetros antes que se realize a experiência podem ser descritos por distribuição de probabilidades, é desejāvel encontrar métodos algé- bricos eficientes e flexíveis para incorporar estas informações na expressão matemática dos problemas de estimação.
RAIFFA e SCHLAIFER (1961) sugerem algumas condições que devem ser observadas na escolha da famỉia F de distribuições a serem utilizadas para representar a distribuição "a priori" em um problema particular:

a) "F deve ser analiticamente tratável em três aspectos: 19)de ve ser razoavelmente fácil determinar a distribuição "a posteriori" resultante de uma dada distribuição "a priori" e dada por uma amostra; 29) deve ser possível expressar em forma conveniente as esperanças matemáticas de funções perda (ou funções utilidade) com respeito a algum membro de F; 39) F deve ser fechada, no sentido de que se "a priori" é membro de F, "a posteriori" também será um membro de F.

b) F deve ser completa, assim existirá um membro de F capaz de expressar a informação e o grau de confiança "a priori" daquele que decide.

c) F deve ser parametrizável, de maneira que possa ser facilmen te interpretada, no sentido de ser fácil verificar que o membro es- 
colhido da famîlia esteja de acordo com o julgamento "a priori" sobre $\theta$ e não um mero artifício concordando com uma ou duas sumarizações quantitativas deste julgamento:"

Nos exemplos de aplicação do método bayesiano desenvolvidos em 3.3., estimando parâmetros em modelos de regressão 1inear, consideraremos as situações em que a distribuição "a priori" representa desconhecimento ou ignorância a respeito dos parâmetros (distribuição "a priori" difusa) e o caso de distribuição "a priori" não difusa, representando o conhecimento que se tem sobre os parâme tros antes de realizar o experimento.

\subsection{2 - 0 conceito de informação contida na amostra}

Como dissemos anteriormente, o método bayesiano,prin cipalmente quando aplicado em tomadas de decisão, utiliza informações extras sobre os parāmetros (além daquelas sobre os apropriados espaços paramétricos e amostral e da função de densidade) e as incorpora na estimação do verdadeiro valor do parâmetro, de maneira explícita pela distribuição "a priori". Nos métodos não bayesianos, a utilização da informação "a priori" se faz de maneira implícita como é, por exemplo, na decisão em delinear este ou aquele tipo de experimento conforme determina a anālise teōrica da situação objetí va em estudo. A diferença fundamental'é que todo o peso da decisão é colocado, no método clássico, na evidência dos dados amostrais. 
Usado ou não como fundamento da tomada de decisão, o resultado de qualquer experimento conduzido dentro de critérios cientỉficos, poderá servir para ampliar nosso conhecimento sobre o estado real da natureza e se prestar para solucionar problemas de delineamentos experimentais,bem como,ser útil em futuras tomadas de decisão. Assim, o estatístico irā delinear, entre vārios experimentos possíveis, aquele que irā fornecer a maior quantidade média de informação.

o conceito e a expressão "quantidade de informação" foram dados inicialmente por R.A. Fisher em 1921, e por ele mesmo de senvolvidos em "Theory of Statistical Estimation" - 1925. Em 1948, estudando problemas ligados à comunicação, Shannon amplia e estabelece uma maneira de medir a informação que LINDLEY (1956) utiliza pa ra introduzir a medida de informação proveniente de um experimento. As conclusões mais importantes deste ültimo trabalho, servirão, de base para a apresentação do que se segue.

A comparação entre os conhecimentos anterior e poste rior à realização de um experimento torna possível medir a quantida de de informação provinda do experimento,e a consideração da distrí buição "a priori" é básica para este estudo. Se a distribuição "a priori" é, por exemplo, concentrada em um valor do parâmetro, ou se ja, se o estado da natureza é conhecido, então nenhum experimento é informativo sobre o parâmetro.

Para a distribuição "a priori", $\pi(\theta)$, a quantidade de 
informąção com relação a d $\theta \vec{e}$ definida por ${ }^{(6)}$ :

$$
I_{0}=\int_{\Omega} \pi(\theta) \ln \pi(\theta) d \theta,
$$

se $\pi(\theta) \neq 0$, e se $\pi(\theta)=0$, define-se integrando igual a zero.

Após o experimento ter sido realizado e o valor de $x$ observado, a quantidade de informação é

$$
I_{1}(x)=\int_{\Omega} \pi(\theta \mid x) \ln \pi(\theta \mid x) d \theta
$$

e se $\pi(\theta \mid x)=0$, define-se o integrando com valor zero.

$$
\text { Uma ṇotação útil para (2.1) e (2.2) se faz usando o }
$$

conceito de esperança matemática:

$$
\begin{aligned}
I_{0} & =E_{\theta}[\ln \pi(\theta)] . \\
I_{1}(x) & =E_{\theta}[\ln \pi(\theta \mid x)]
\end{aligned}
$$

Nota-se que a medida do conteúdo informativo depende da base do logaritmo. As unidades mais comumente usadas são: "bit", se a base do logaritmo è dois e "nit" se usarmos o logaritmo natural. Empregaremos este ūltimo em nossas considerações e é fácil observar que:

$$
\begin{aligned}
& 1 \mathrm{bit}=0,693 \mathrm{nit} \\
& 1 \mathrm{nit}=1,443 \mathrm{bit}
\end{aligned}
$$

(6) Un estudo introdutório sobre como chegar a esta expressão analỉ tiça pode ser feito em HOFFMANN (1980), päg. 287, e de maneira bem prática e ilustrativa em PUGACHEV (1973), pág. 278, que define a medida quantitativa de indeterminação de fenômenos aleatórios no conceito de Entropia do experimento. 
40 .

DEFINIÇÃo 1: A quantidade de informação provinda de um experimento e, com conhecimento "a priori" $\pi(\theta)$, quando a observação è $x$, è definida:

$$
\mathrm{I}[\mathrm{e} ; \pi(\theta) ; \mathrm{x}]=\mathrm{I}_{1}(\mathrm{x})-\mathrm{I}_{0}
$$

DEFINIÇÃo 2: A quantidade média de informação provinda do experimen to $\underline{e}$, com conhecimento "a priori" $\pi(\theta)$, é:

$$
I(e)=I[e ; \pi(\theta)]=E_{x}\left[I_{1}(x)-I_{0}\right] \text {. }
$$

Esta ültima expressão pode ser escrita por outras ma neiras também úteis. Por (2.3) e (2.4) teremos:

$$
\begin{aligned}
I(e) & =E_{x}\left\{E_{\theta}[\ln \pi(\theta \mid x)]-E_{\theta}[\ln \pi(\theta)]\right\} \\
& =E_{x}\left\{E_{\theta}[\ln \pi(\theta \mid x)-\ln \pi(\theta)]\right\} \\
I(e) & =E_{x}\left\{E_{\theta}\left[\ln \frac{\pi(\theta \mid x)}{\pi(\theta)}\right]\right\}
\end{aligned}
$$

Pela expressão (1.2) do teorema de Bayes, (2.7) tambēm pode se apresentar na forma:

$$
I(e)=E_{x}\left\{E_{\theta}\left[\ln \frac{p(x \mid \theta)}{p(x)}\right]\right\}
$$

Por outro 1 ado, se $\mathrm{p}(\mathrm{x} \mid \theta)$ é a densidade conjunta para $x$ e $\theta$, então: 
41.

$$
p(x ; \theta)=p(x \mid \theta) \pi(\theta)
$$

e

$$
p(x \mid \theta)=\frac{p(x ; \theta)}{\pi(\theta)},
$$

e teremos:

$$
\begin{gathered}
I(e)=E_{x}\left\{E_{\theta}\left[\ln \frac{p(x ; \theta)}{p(x) \pi(\theta)}\right]\right\} \\
I(e)=\int_{S} \int_{\Omega} p(x ; \theta) \text { \&n }\left[-\frac{p(x ; \theta)}{p(x) \pi(\theta)}\right] d \theta d x
\end{gathered}
$$

Utilizando o operador informação I com o operador de esperança matemática E, Lindley introduz uma expressão ūtil em cá1culos para (2.9).

Definindo a informação para uma função de densidade $\mathrm{p}(\mathrm{x})$ :

$$
I_{x} p(x)=\int_{S} p(x) \ln p(x) d x
$$

teremos :

$$
I(e)=E_{\theta} I_{x} p(x \mid \theta)-I_{x} E_{\theta} p(x \mid \theta)
$$

DEFINIÇÃo 3: Dois experimentos, $e_{1}$ e $e_{2}$, com $\Omega_{1}=\Omega_{2}=\Omega$, são inde pendentes se $p\left(x_{1}, x_{2} \mid \theta\right)=p\left(x_{1} \mid \theta\right) \cdot p\left(x_{2} \mid \theta\right) \quad p$ ara todo $\theta \in \Omega$. 
DEFINIÇÃo 4: Sejam e e e e dois experimentos com $\Omega_{1}=\Omega_{2}=\Omega$. Dizemos que $e_{1}$ é mais informativo que $e_{2}$ e, para toda $\pi(\theta)$ :

$$
I\left[\underline{e}_{1} ; \pi(\theta)\right] \geq I\left[e_{2} ; \pi(\theta)\right],
$$

o que mostra que o mérito de cada experimento só pode ser julgado pela referência à distribuição "a priori".

Seguem, sem demonstração, algumas propriedades sô bre a medida da informação provinda de um experimento, a maioria das quais, desenvolvidas no trabalho de Lindley citado, em forma de teo remas.

Propriedades :

a) $I(e) \geq 0$, com igualdade somente se $p(x \mid \theta)$ não depende de $\theta$.

b) Se $e_{1}$ e $e_{2}$ são dois experimentos e e $=\left(e_{1} ; e_{2}\right)$, chamacio de soma dos experimentos, então:

$$
I(e)=I\left(e_{1}\right)+I\left(e_{2} \mid e_{1}\right) .
$$

c) Se $x_{1}$ é suficiente para $x$ (no sentido de Neyman-Fisher da fa torização), então $I(e)=I\left(e_{1}\right)$. Em palavras, não haverá per da de informação se concentrarmos nossa atenção à observação da estatística suficiente.

d) Se $e_{1}$ e $e_{2}$ são experimentos independentes, então

$$
I\left(e_{2} \mid e_{1}\right) \leq I\left(e_{2}\right),
$$


ou seja, um deles é, em média, mais inf̣ormativo se realizado anteriormente ao outro.

e) A informação contida na amostra não pode ser aumentada por uma operação estatística e é invariante se, e somente se, for empregada uma estatística suficiente.

E interessante apresentar o conceito de Entropia de uma variável aleatória, que pode ser visto de maneira acessível em PUGACHEV (1973). A Entropia (H) é tomada como medida quantitativa da indeterminação de uma prova, e define-se:

$$
H=-\int_{S} p(x) \ln p(x) d x
$$

ou,

$$
H=-E[\ln p(x)],
$$

onde $p(x)$ representa a f.d.p. da variável aleatória contínua $x$.

Na prática, para se eliminar a indeterminação de uma variável aleatória é necessārio efetuar um experimento e determinar o valor que esta toma. Na verdade, a variável aleatôria $\mathrm{X}$ que nos in teressa não é passível de observação, o que nos leva a tirar conclu sões dela a respeito de outras variáveis aleatórias, ou seja, obser var variáveis aleatórias que contenham informação a respeito de $\mathrm{x}$. A entropia condicional média de X com relação a $Y$ se determina pe1a fórmula:

$$
H_{y}[x]=-\int_{-\infty}^{\infty} \int p(x ; y) \ln p_{1}(x \mid y) d x d y,
$$


ou,

$$
H_{y}[X]=-E[\ln \quad \mathrm{p}(\mathrm{x} \mid \mathrm{Y})]
$$

E fácil verificar que se $X$ e $Y$ são variáveis aleatórias independentes, então

$$
\mathrm{H}_{\mathrm{y}}[\mathrm{X}]=\mathrm{H}[\mathrm{x}],
$$

o que mostra que as variáveis aleatórias independentes de X não podem conter nenhuma informação sobre $\mathrm{X}$.

Definimos a Entropia conjunta das variáveis $X$ e $Y$ :

$$
\mathrm{H}[\mathrm{X} ; \mathrm{Y}]=\mathrm{H}[\mathrm{X}]+\mathrm{H}_{\mathrm{X}}[\mathrm{Y}]=\mathrm{H}[\mathrm{Y}]+\mathrm{H}_{\mathrm{y}}[\mathrm{X}] \text {, }
$$

e se elas são independentes:

$$
\mathrm{H}[\mathrm{X} ; \mathrm{Y}]=\mathrm{H}[\mathrm{X}]+\mathrm{H}[\mathrm{Y}] \text {. }
$$

Notemos que a diminuição na Entropia da variável $\mathrm{X}$, obtida como resultado da observação da váriável $Y$, pode ser tomada como medida da quantidade de informação sobre $X$, contida em $Y$. E podemos escrever:

$$
\begin{gathered}
\mathrm{I}_{\mathrm{y}}[\mathrm{X}]=\mathrm{H}[\mathrm{X}]-\mathrm{H}_{\mathrm{y}}[\mathrm{X}] . \\
\text { Como } \mathrm{H}[\mathrm{X} ; \mathrm{Y}]=\mathrm{H}[\mathrm{X}]+\mathrm{H}_{\mathrm{X}}[\mathrm{Y}]=\mathrm{H}[\mathrm{Y}]+\mathrm{H}_{\mathrm{y}}[\mathrm{X}], \\
\mathrm{H}[\mathrm{X}]-\mathrm{H}_{\mathrm{y}}[\mathrm{X}]=\mathrm{H}[\mathrm{Y}]-\mathrm{H}_{\mathrm{X}}[\mathrm{Y}] \\
\mathrm{I}_{\mathrm{y}}[\mathrm{X}]=\mathrm{I}_{\mathrm{X}}[\mathrm{Y}] .
\end{gathered}
$$


Esta $\bar{u} 1$ tima igualdade mostra que se as variáveis $X$ e Y são dependentes, cada uma delas contēm uma quantidade positiva de informação sobre a outra.

Finalmente citaremos três propriedades sobre a Entro pia de variáveis aleatórias, que justificam, sob este aspecto, o uso frequente de certas distribuições ${ }^{(7)}$ :

19) Entre todas as variáveis aleatórias que têm a mesma disper são, aquelas normalmente distribuídas são as que possuem a maior En tropia.

20) Entre todas as variáveis aleatórias contínuas e positivas, com a mesma esperança matemātica, as que possuem maior Entropia são aquelas distribuidas pela lei exponencial.

39) Entre todas as distribuições com mesmo intervalo 1 imitado de valores possíveis para a variável aleatória, a distribuição uniforme neste intervalo é a que possui maior Entropia.

Por outro lado, a quantidade de informação de Fisher sobre $\theta$ (designada aqui por $i_{\theta}$ ), contida em uma observação $X \in S$, é dada (para certas condições de regularidade quanto à diferenciação sob o sinal da integral) por:

$$
i_{\theta}=E_{\theta}\left\{\frac{\partial \log p(x \mid \theta)}{\partial \theta}\right\}^{2}
$$

(7) Uma demonstração da 1 a propriedade pode ser vista em PUGACHEV (1973), pág. 291. 
ou

$$
i_{\theta}=-E_{\theta}\left[\frac{\partial^{2} \log p(x \mid \theta)}{\partial \theta^{2}}\right] .
$$

No caso de $\theta$ ser um vetor de $\mathrm{k}$ parâmetros, teremos a matriz de informação de Fisher. $\mathbb{E}$ a matriz de ordem $k x k$, cujo $(i, j)-$ -ésimo elemento é:

$$
E_{\theta}\left[\frac{\partial \log p(x \mid \theta)}{\partial \theta_{i}} \cdot \frac{\partial \log p(x \mid \theta)}{\partial \theta_{j}}\right]=-E_{\theta}\left[\frac{\partial^{2} \log p(x \mid \theta)}{\partial \theta_{i} \partial \theta_{j}}\right]
$$

Lembramos que esta definição è utilizada na conhecida desigualdade de Cramér-Rao para a variância de um estimador não tendencioso de $\theta$ :

$$
\operatorname{var}(\hat{\theta}) \geq \frac{1}{\mathrm{E}_{\theta}[\partial \log \mathrm{p}(\mathrm{x} \mid \theta) / \partial \theta]^{2}}=\frac{1}{i_{\theta}}
$$

LINDLEY (1957) observou as diferenças entre o concei to de informação de Fisher e aquele desenvolvido por Shannon. Além daquela fundamental de que Fisher não usou a distribuição "a priori" do parâmetro desconhecido, o conceito de Shannon, em esquemas amostrais, utiliza a verossimilhança do ponto amostral, considerando $\theta$ como variável aleatória. Por outro lado, Lindley verifica a equivalência entre ambos quando aplicados a esquemas amostrais de informa ção constante e a equivalência assintótica entre a variância "a pos teriori" de $\theta$ e a variância de um estimador assintoticamente eficien te $\operatorname{de} \theta$. 
47 .

\subsection{3 - Ausēncia de informação "a priori"}

Em algumas situações podemos sentir que não existe ne nhuma informação "a priori" sobre o estado real da natureza. 0 esquema bayesiano deve considerar também este caso e selecionar distribuições "a priori" que representem desconhecimento ou "ignoorância" sobre os parâmetros para utilizar a expressão (1.2) na determi nação da distribuição "a posteriori".

Praticamente toda bibliografia consultada nos mostra que, embora com algumas variações em casos muito específicos, este problema tem sido resolvido com base em duas regras estabelecidas e discutidas por JEFFREYS (1948). Um dos casos ilustrativos utilizado neste trabalho, será baseado nestas regras que passamos a apresentar.

REGRA 1: Se temos completa "ignorância" sobre o valor do parâmetro, que pode ter valores em um intervalo finito ou em toda extensão do campo real, a sua distribuição "a priori" pode ser tomada como uniforme neste intervalo.

REGRA 2: Se a nossa "ignorância" è a respeito de um parâmetro que pode assumir valores de 0 a $+\infty$, a distribuição "a priori" do seu logaritmo pode ser tomada como uniforme neste intervalo. 
A Regra 1 pode englobar casos como, por exemplo, a média de uma distribuição normal $(-\infty<\mu<+\infty)$ ou de uma distribuição binomial $(0 \leq \mathrm{P} \leq 1)$, e representa-se nas formas:

$$
\pi(\theta) d \theta \propto d \theta
$$

ou

$$
\pi(\theta) \propto \text { constante }
$$

para $-\infty<\theta<+\infty$ ou $-k \leq \theta \leq k$.

Esta f.d.p. pode ser imprópria, pois $\int_{-\infty}^{\infty} \pi(\theta) d \theta=\infty$ e, neste caso, tomando-se $-\infty<\theta<+\infty$ como uma afirmação certa, Jef freys utilizou o símbolo $\infty$ para representar a probabilidade de um evento certo, ao invés de 1 . No ponto de vista de Jeffreys, sendo $\underline{a}, \underline{b}, \underline{c}$ e $\underline{d}$ números reais finitos, então teremos indeterminação na razão:

$$
\frac{P(a<\theta<b)}{P(c<\theta<d)}
$$

e, por isto, não podemos fazer nenhuma afirmação de que $\theta$ esteja em um particular par de intervalos, tomando então (2.14) como represen tação de "ignorância".

$$
\text { Para }-k \leq \theta \leq k \text {, podemos escrever a f.d.p. própria, }
$$

desde que façamos:

$$
p(\theta) d \theta=\frac{1}{2 k} d \theta
$$




\section{Neste caso, a razão}

$$
\frac{P(a<\theta<b)}{P(c<\theta<d)}=\frac{\frac{b-a}{2 k}}{\frac{d-c}{2 k}}=\frac{b-a}{d-c}
$$

é determinada, para $\underline{a}, \underline{b}, \underline{c}$ e $\underline{\mathrm{d}}$ números reais finitos do intervalo $[-k ; k]$. Podemos considerar que para $\underline{k}$ tendo alto valor, tem-se uma aproximação para a forma (2.14) desde que a razão

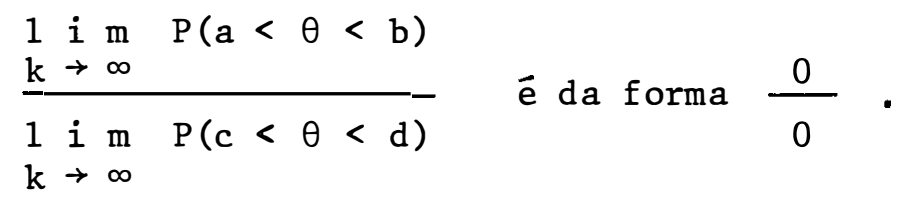

Por outro lado, se tomarmos a medida de informação para uma f.d.p., usada para concluir (2.10)

$$
I_{\theta} \pi(\theta)=\int_{-k}^{k} \pi(\theta) \ln \pi(\theta) d \theta
$$

pode-se verificar que a informação mínima a respeito do parâmetro $\theta$ ocorre quando se faz uso da f.d.p. própria em (2.15).

Para demonstrar isto devemos minimizar a informação na condição de que:

$$
\int_{-k}^{k} \pi(\theta) d \theta=1
$$

Pelo método dos multiplicadores de Lagrange, podemos estabelecer:

$$
\phi=I_{\theta} \pi(\theta)+\lambda\left[\int_{-k}^{k} \pi(\theta) d \theta-1\right]
$$




$$
\phi=\int_{-k}^{k} \pi(\theta) \ln \pi(\theta) d \theta+\lambda \int_{-k}^{k} \pi(\theta) d \theta-\lambda
$$

Derivando em relação a $\pi(\theta)$ e igualando a zero teremos a condição para minimižação:

$$
\frac{\partial \phi}{\partial \pi(\theta)}=1+\ln \pi(\theta)+\lambda=0
$$

ou

$$
\begin{aligned}
\ln \pi(\theta) & =-(\lambda+1) \\
\pi(\theta) & =e^{-(\lambda+1)}
\end{aligned}
$$

Substituindo na condição, encontramos:

$$
\lambda+1=\ln 2 \mathrm{~K},
$$

então

$$
\pi(\theta)=e^{-\ln 2 \mathrm{~K}}=\mathrm{e}^{\ln 1 / 2 \mathrm{~K}},
$$

ou seja,

$$
\pi(\theta)=\frac{1}{2 \mathrm{~K}},
$$

que representa a f.d.p. de (2.15)。

Deve-se ressaltar que o uso da f.d.p. impröpria para a distribuição "a priori" indicando "ignorância", combinada com a verossimilhança da amostra, através do teorema de Bayes, normalmente fornece f.d.p. "a posteriori" própria e informativa sobre os parâme tros.

A Regra 2 de Jeffreys pode ser aplicada no caso de o parâmetro (por exemplo a variância) assumir valores de 0 a $+\infty$.Nes te caso assumimos que $\phi=\ln \theta$ é uniformemente distribuỉdo, ou seja: 


$$
\pi(\phi) \mathrm{d} \phi \propto \mathrm{d} \phi \quad,
$$

uma vez que $0<\theta<+\infty$ tem-se $-\infty<\phi<+\infty$ e recai-se na análise da regra 1.

$$
\begin{aligned}
& \text { Como } \begin{aligned}
\mathrm{d} \phi=\frac{\mathrm{d} \theta}{\theta} & \text {, podemos escrever: } \\
\pi(\theta) \mathrm{d} \theta \propto \frac{\mathrm{d} \theta}{\theta} \quad(0<\theta<+\infty) & (2.16)
\end{aligned}
\end{aligned}
$$

De maneira semelhante ao que foi feito para a Regra 1 , podemos demonstrar que $\mathrm{p}(\phi) \propto$ constante é a condição para que a informação seja minimizada, o que é uma justificativa teórica de que podemos tomar $\phi=\ln \theta$ como uniformemente distribuída para indicar nosso desconhecimento a respeito de $\theta$ e que implica em (2.16).

Finalmente, ainda a respeito do estudo de Jeffreys so bre distribuição "a priori", representando pouco conhecimento ou "ig norância" acerca dos parâmetros, podemos citar, una forma geral de f.d.p. "a priori", que é consistente em relação a propriedades de in variância ${ }^{(8)}$.

Sendo $\theta$ um vetor de parâmetros, a f.d.p. "a priori"

é tomada como:

$$
\pi(\theta) \propto\left|i_{\theta}\right|^{1 / 2}
$$

onde $i_{\theta} \bar{e}$ a matriz de informação de Fisher, apresentada ere (2.12) e (2.13).

Jeffreys mostrou que a forma (2.17) é invariante no

(8) Além de JEFFREYS (1948), uma exposição detalhada pode ser vista em HARTIGAN (1964). 
sentido de que se um modelo foi parametrizado em termos do vetor $\mu$, onde $\mu=F(\theta)$, com $F$ sendo uma transformação diferenciável dos ele mentos de $\theta$ um a um, as afirmações probabilisticas "a posteriori" se rão consistentes em relação às do modelo parametrizado em $\theta$. Seria o caso, por exemplo, de se trabalhar com o desvio padrão $(\sigma)$, a variância $\left(\sigma^{2}\right)$ ou a precisão $\left(h=\sigma^{-2}\right)$.

A f.d.p. de (2.17) representa uma forma útil para in dicar desconhecimento "a priori", o que pode ser mostrado en termos da teoria da informação.

A informação sobre $\theta$, contida em $p(x \mid \theta)$ é definida:

$$
I_{x}(\theta)=\int_{S} p(x \mid \theta) \cdot \ln p(x \mid \theta) d x
$$

e a informação média "a priori":

$$
\bar{I}_{x}=\int_{\Omega} I_{x}(\theta) \pi(\theta) d \theta .
$$

Definimos o ganho de informação:

$$
\begin{aligned}
& G=\bar{I}_{x}-\int_{\Omega} \pi(\theta) \ln \pi(\theta) d \theta \\
& G=\int_{\Omega} I_{x}(\theta) \pi(\theta) d \theta-\int_{\Omega} \pi(\theta) \ln \pi(\theta) d \theta .
\end{aligned}
$$

Assim, para adotarmos uma determinada f.d.p. própria como sendo "a priori" que represente "ignorância" sobre os parâmetros, devemos tomar aquela que maximize $G$, sujeito a $\int_{\Omega} \pi(\theta) d \theta=1$. Entretanto,verifica-se que a chamada "priori" invariante de Jeffreys em (2.17) nem sempre maximiza $G^{(9)}$. Se tomarmos $G^{\prime}$ como forma assin-

(9) Em ZELLNER (1971), pāg. 51, podem ser vistos dois exemplos em dis tribuição normal, onde a referida priori de Jeffreys maximiza $\bar{G}$ e un exemplo onde isto não ocorre. 
tótica de $G$, verificamos que (2.17) satisfaz à condição de maximizâ ção :

$$
G^{\prime}=\int_{\Omega} \pi(\theta) \ln \sqrt{n}\left|i_{\theta}\right|^{1 / 2} d \theta-\int_{\Omega} \pi(\theta) \ln \pi(\theta) d \theta,
$$

onde $\underline{n}$ é o número de observações independentes de $p(x \mid \theta)$ 。

Para concluir, podemos dizer que, além de satisfatória para a maioria dos casos, as Regras de Jeffreys consideram distribui ção "a priori" uniforme que atende às condições de Raiffa-Schlaifer apresentadas em 3.2 .1 .

\subsection{4 - Distribuições conjugadas}

Como foi visto em 3.2 .1 , o conhecimento anterior ao experimento deve ser expresso pela f.d.p. da distribuição "a priori" que atenda a algumas condições que facilitem,não só o tratamento al gébrico, como também traduzam o nosso grau de confiança a respeito de afirmações probabilísticas sobre o parâmetro. Os mesmos autores, RAIFFA e SCHLAIFER (1961), propõem o uso de Distribuições Conjugadas para atender àquelas condições.

Basicamente, a idêia utilizada por estes autores é a de usar como núcleo ("kernel") da f.d.p. "a priori" o mesmo núcleo da função de verossimilhança amostral. Desta forma, se a distribuição "a posteriori" apresentar os mesmos parâmetros e pertencer àmes ma famỉlia de distribuições de probabilidade, dizemos que a famỉlia é conjugada em relação ao modelo usado na amostra atual. 
Sendo D uma função de densidade de $\theta$, e K uma outra função de $\theta$, tal que

ou seja, a razão $\frac{K(\theta)}{D(\theta)}=\int_{\Omega} K(\theta) d \theta$ é constante em relação a $\theta$, podemos escrever:

$$
\mathrm{D}(\theta)=\frac{\mathrm{K}(\theta)}{\int_{\Omega} \mathrm{K}(\theta) \mathrm{d} \theta},
$$

$$
\mathrm{D}(\theta) \propto \mathrm{K}(\theta)
$$

e dizemos que $\mathrm{K}$ é um nücleo ("kernel") da densidade de $\theta$ 。 Assim, se a verossimilhança de $x$ dado $\theta \bar{e}$ fornecida por

$$
p(x \mid \theta)=k(x \mid \theta) \cdot \rho(x), \quad \text { para todo } x \text { e } \theta \text {, }
$$

dizemos que $k(x \mid \theta)$ é o núcleo da verossimilhança de $x$, dado $\theta$ e $\rho(x)$ é o "resỉduo" desta verossimilhança.

Pelo teorema de Bayes $(1.2)$ e sendo $K^{\top}(\theta)$ o núcléo da f.d.p. "a priori", podemos escrever:

$$
\pi(\theta \mid x)=\pi(\theta) p(x \mid \theta) N(x)
$$

$\operatorname{com} N(x)=[p(x)]^{-1}$

$$
\begin{gathered}
\text { Então } \pi(\theta \mid x)=K^{\prime}(\theta)\left[\int_{\Omega} k^{\prime}(\theta) d \theta\right]^{-1} k(x \mid \theta) \rho(x) N(x) \\
\pi(\theta \mid x) \propto K^{\prime}(\theta) k(x \mid \theta) .
\end{gathered}
$$

Consideremos tambēm $z=\tilde{z}(x)$ como um conjunto de estatísticas calculadas a partir dos dados amostrais。 Se $\pi(\theta \mid z)=\pi(\theta \mid x)$ 
então $z$ é um conjunto de estatísticas suficientes (10).

TEOREMA $(11)$ : Sejam $z_{(1)}$ e $z_{(2)}$ dois conjuntos de estatisticas suficientes que descrevem um modelo de amostragem, tal que $z_{(1)}=z_{p}\left(x_{1} ; \ldots ; x_{p}\right)$ e $z_{(2)}=z_{n-p}\left(x_{p+1} ; \ldots ; x_{n}\right)$, en tão é possîvel encontrar uma operação binária* tal que $z_{*}=z_{(1)} * z_{(2)}$ apresenta as propriedades:

a) $\mathrm{p}_{\mathrm{n}}\left(\mathrm{x}_{1} ; \ldots ; \mathrm{x}_{\mathrm{n}} \mid \theta\right) \propto \mathrm{k}\left(\mathrm{z}_{*} \mid \theta\right)$;

b) $k\left(z_{*} \mid \theta\right) \propto k\left(z_{(1)} \mid \theta\right) \cdot k\left(z_{(2)} \mid \theta\right)$ 。

Assim, se $z=\tilde{z}(x)$ è uma estatística suficiente; se $k(z \mid \theta)$ è o núcleo da verossimilhança de $x$ dado $\theta$ e se $\pi(\theta)=k\left(z^{p} \mid \theta\right)$, então o teorema de Bayes fornecerá a f.d.p. "a posteriori" definida por:

$$
\pi(\theta \mid z) \propto \pi(\theta) k(z \mid \theta)
$$

ou

$$
\pi(\theta \mid z) \propto k\left(z^{\prime} \mid \theta\right) \cdot k(z \mid \theta) \propto k\left(z_{*} \mid \theta\right),
$$

ou seja, o núcleo da densidade "a priori" combina com o núcleo da f.d.p. da amostra "atual" da mesma maneira como se combinam dois nū cleos das densidades amostrais. Além disso, se obtēm simplificação considerāvel quando se usam estatísticas suficientes que resumem to da informação relevante, contida na amostra.

(10) 0 conceito bayesiano de estatística suficiente e a comparação com o conceito de Fisher-Neyman, pode ser encontrado em LINDLEY (1965), págs. 46-58 è em FERGUSON (1969), Capitulo 3.

(11) A demonstração deste teorema pode ser vista em RAIFFA e SCHALAI FER (1961), pág. 45. 
3.3 - Aplicações em Modelo de Regressão Linear Simples

\subsection{1 - 0 modelo e pressuposições}

Faremos aplicação do método bayesiano de estimação por pontos na situação em que se observa uma dependência linear de uma variável aleatória $Y$ em função de uma variável não-àleatória $X$, o que pode ser estabelecido pelo modelo estatistico:

$$
\mathrm{Y}_{i}=A+\beta \mathrm{X}_{i}+\mathrm{u}_{i} \quad(\mathrm{i}=1,2, \ldots, \mathrm{n}) .
$$

Ao estabelecermos o modelo, e dispondo de $\underline{\text { n }}$ pares de valores das variáveis, pressupomos que:

I) Os valores da variāvel independente, $\mathrm{X}$, são fixos (não-alea tórios);

II) Os erros $u_{i}$ são independentes e normalmente distribuỉdos, com média zero e variância $\sigma^{2}$ (chamada de variância residua1).

Assim, podemos escrever:

$$
\begin{aligned}
u_{i} & \cap N\left(0 ; \sigma^{2}\right) \\
E\left(u_{i}\right) & =0 \\
E\left(u_{i}^{2}\right) & =E\left[Y_{i}-A-B X_{i}\right]^{2}=\sigma^{2} \\
E\left(u_{i}, u_{j}\right) & =0, \text { para } i \neq j
\end{aligned}
$$

Para simplificação dos cálculos, podemos utilizar a variävel centrada $x_{i}=x_{i}-\bar{x}$, o que corresponderia, graficamente, a tomar a média de $\mathrm{X}_{\mathrm{i}}$ como origem dos eixos das abcissas. Desta forma 
o modelo estatỉstico fica:

$$
Y_{i}=\alpha+\beta\left(X_{i}-\bar{X}\right)+u_{i}
$$

ou

$$
\mathrm{Y}_{\mathbf{i}}=\alpha+\beta \mathrm{x}_{\mathbf{i}}+\mathrm{u}_{\mathbf{i}}
$$

De acordo com a notação introduzida anteriormente, de

finimos:

$\pi(\theta)=\pi\left(\alpha ; \beta ; \sigma^{2}\right):$ f.d.p. da distribuição "a priori", onde $\theta$ é o vetor aleatório dos parâmetros; $p(y \mid \theta)$ : função de verossimilhança da amostra aleatória

$$
\begin{gathered}
y=\left(y_{1}: y_{2} ; \ldots ; y_{n}\right) \\
\pi(\theta \mid y)=\pi\left(\alpha ; \beta ; \sigma^{2} \mid y\right): f_{\circ} d \circ p \cdot \text { da distribuição "a posterior }{ }^{n "} \text {. }
\end{gathered}
$$

3.3.2 - Uso da f.d.p. indicando ausēncia de informação "a priori"

Esta primeira aplicação é interessante para comparar os resultados com os obtidos pelos métodos usuais de Mínimos Quadra dos e da Māxima Verossimilhança, que não consideram conhecimento so bre os parâmetros anterior à realização do experimento.

Pelo método bayesiano, o desconhecimento ou "ignorância" "a priori" sobre os parâmetros (considerados variáveis aleatōrias) deve ser evidenciado na expressão (1.2)。Utilizaremos, para este caso, a f.d.p. "a priori", de acordo com as regras de Jeffreys, expressas por $(2.14)$ e $(2.16)$. 
Assim, para $\alpha$ e $\beta,-\infty<\alpha ; \beta<+\infty$, consideramos a f.d.p. "a priori":

$$
\begin{aligned}
& \pi(\alpha) \mathrm{d} \alpha=\mathrm{k}_{1} \mathrm{~d} \alpha \text { ou } \pi(\alpha) \mathrm{d} \alpha \propto \mathrm{d} \alpha \\
& \pi(\beta) \mathrm{d} \beta=\mathrm{k}_{2} \mathrm{~d} \beta \text { ou } \pi(\beta) \mathrm{d} \beta \propto \mathrm{d} \beta \\
& \text { Como } 0<\sigma^{2}<+\infty \text {, tomamos } \phi=\ln \sigma^{2} \text { e teremos } \\
& -\infty<\phi<+\infty \quad \text { e a f.d.p. serä: } \\
& \pi(\phi) \mathrm{d} \phi=\mathrm{k}_{3} \mathrm{~d} \phi \text { ou } \pi(\phi) \mathrm{d} \phi \propto \mathrm{d} \phi \\
& \text { Sendo } \mathrm{d} \phi=\frac{\mathrm{d} \sigma^{2}}{\sigma^{2}}, \text { teremos: } \\
& \pi\left(\sigma^{2}\right) \mathrm{d} \sigma^{2}=\mathrm{k}_{3} \frac{\mathrm{d} \sigma^{2}}{\sigma^{2}} \text { ou } \pi\left(\sigma^{2}\right) \mathrm{d} \sigma^{2} \propto \frac{\mathrm{d} \sigma^{2}}{\sigma^{2}}
\end{aligned}
$$

Considerando $\alpha, \beta$ e $\sigma^{2}$ independentemente distribuidos, a f.d.p. "a priori" será:

$$
\pi\left(\alpha ; \beta ; \sigma^{2}\right)=k_{1} k_{2} k_{3} \frac{1}{\sigma^{2}} \text { ou } \pi\left(\alpha ; \beta ; \sigma^{2}\right) \propto \frac{1}{\sigma^{2}}
$$

Pelas pressuposições do modelo, os $\mathrm{Y}_{\mathbf{i}}$ (dados os $\mathrm{x}_{\mathbf{i}}$, $\alpha, \beta$ e $\sigma^{2}$ ) serāo normal e independentemente distribuỉdos, com

$$
E\left(Y_{i} \mid x_{i} ; \alpha ; \beta ; \sigma^{2}\right)=\alpha+\beta x_{i}
$$

e

$$
\begin{aligned}
& V\left(Y_{i} \mid x_{i} ; \alpha ; \beta ; \sigma^{2}\right)=\sigma^{2} \text { ou } \quad V\left(Y_{i} \mid x_{i} ; \theta\right)=\sigma^{2} \\
& \text { Desta forma a Função de Verossimilhança fica: } \\
\mathrm{P}\left(\mathrm{y} \mid \mathrm{x} ; \alpha ; \beta ; \sigma^{2}\right)= & \left(2 \pi . \sigma^{2}\right)^{-\mathrm{n} / 2} \text { exp }\left\{-\frac{1}{2 \sigma^{2}} \sum_{i=1}^{n}\left[\mathrm{Y}_{i}-\alpha-\beta\left(\mathrm{x}_{i}-\overline{\mathrm{X}}_{i}\right)\right]^{2}\right\}
\end{aligned}
$$


Para se determinar a f.d.p。 "a posteriori" para os parāmetros, utilizamos a fórmula de Bayes:

$$
\pi\left(\alpha ; \beta ; \sigma^{2} \mid y\right)=\frac{\pi\left(\alpha ; \beta ; \sigma^{2}\right) \cdot p\left(y \mid \alpha ; \beta ; \sigma^{2}\right)}{p(y)} .
$$

ou, $\operatorname{com} \dot{\theta}=\left(\alpha ; \beta ; \sigma^{2}\right)^{\urcorner}$:

$$
\pi(\theta \mid y)=\frac{\pi(\theta) \cdot p(y \mid \theta)}{p(y)} \text { ou } \pi(\theta \mid y)=k \cdot \pi(\theta) \cdot p(y \mid \theta)
$$

onde,

$$
\left.\mathrm{k}^{-1}=\mathrm{p} f \mathrm{y}\right)=\int_{\Omega} \pi(\theta) \mathrm{p}(\mathrm{y} \mid \theta) \mathrm{d} \theta
$$

Para obtermos $\mathrm{k}=[\mathrm{p}(\mathrm{y})]^{-1}$ escrevemos a densidade con junta $p\left(y ; \alpha ; \beta ; \sigma^{2}\right)$ e integramos em relação aos parâmetros obtendo a densidade margina1 $\mathrm{p}(\mathrm{y})$ :

$$
\begin{gathered}
\mathrm{p}\left(\mathrm{y} ; \alpha ; \beta ; \sigma^{2}\right)=\pi\left(\alpha ; \beta ; \sigma^{2}\right) \cdot \mathrm{p}\left(\mathrm{y} \mid \alpha ; \beta ; \sigma^{2}\right) \\
\mathrm{p}\left(\mathrm{y} ; \alpha ; \beta ; \sigma^{2}\right)=\frac{\mathrm{k}_{4}}{\sigma^{2}}(2 \pi)^{-\mathrm{n} / 2}\left(\sigma^{2}\right)^{-\mathrm{n} / 2} \cdot \exp \left\{-\frac{1}{2 \sigma^{2}} \sum_{i=1}^{\mathrm{n}}\left[\mathrm{Y}_{\mathrm{i}}-\alpha-\beta x_{i}\right]^{2}\right\} \\
\left(\mathrm{k}_{4}=\mathrm{k}_{1} \mathrm{k}_{2} \mathrm{k}_{3}\right), \\
\mathrm{p}\left(\mathrm{y} ; \alpha ; \beta ; \sigma^{2}\right)=\mathrm{k}_{4}(2 \pi)^{-\mathrm{n} / 2}\left(\sigma^{2}\right)^{-1 / 2}(\mathrm{n}+2) \exp \left\{-\frac{1}{2 \sigma^{2}} \sum_{i=1}^{n}\left[Y_{i}-\alpha-\beta x_{i}\right]^{2}\right\}(3)
\end{gathered}
$$

Por simplicidade, a menos que traga dưvidas, deixare mos de escrever o indice do somatório. Façamos, também:

$$
\begin{aligned}
& s_{x x}=\Sigma\left(x_{i}-\bar{X}\right)^{2}=\Sigma x_{i}^{2} \\
& s_{y y}=\Sigma\left(y_{i}-\bar{Y}\right)^{2}=\Sigma y_{i}^{2}
\end{aligned}
$$


60 .

$$
\begin{gathered}
s_{x y}=\Sigma\left(x_{i}-\bar{x}\right)\left(Y_{i}-\bar{Y}\right)=\Sigma x_{i} y_{i} \\
s^{2}=\Sigma y_{i}^{2}-\frac{\left[\sum x_{i} y_{i}\right]^{2}}{\Sigma x_{i}^{2}} \text { ou } s^{2}=s_{y y}-\frac{s_{x y}^{2}}{s_{x x}} \\
a=\bar{Y}=\frac{1}{n} \sum Y_{i} \quad \text { e } \quad b=\frac{\sum x_{i} y_{i}}{\sum x_{i}^{2}}=\frac{s y}{s_{x x}}
\end{gathered}
$$

Com as notações em (3.9), o somatório do expoente da expressão (3.8) pode ser escrito de forma mais adequada:

$$
\begin{aligned}
& { }^{n}\left[Y_{i}-\alpha-\beta x_{i}\right]^{2}=\sum^{n}\left[\left(Y_{i}-\bar{Y}\right)+(\bar{Y}-\alpha)-\beta x_{i}\right]^{2} \\
& =\Sigma y_{i}^{2}+n(\bar{Y}-\alpha)^{2}+\beta^{2} \sum x_{i}^{2}+2(\bar{Y}-\alpha) \sum\left(Y_{i}-\bar{Y}\right)-2 \beta \sum x_{i} y_{i}- \\
& -2 \beta(\overline{\mathrm{Y}}-\alpha) \quad \Sigma \mathrm{x}_{i} \\
& =s_{y y}+n(\alpha-a)^{2}+\beta^{2} s_{x x}-2 \beta s_{x y} \\
& =s_{y y}+n(\alpha-a)^{2}+s_{x x}\left(\beta^{2}-2 \beta \frac{s_{x y}}{s_{x x}}+\frac{s_{x y}^{2}}{s_{x x}^{2}}-\frac{s_{x y}^{2}}{s_{x x}}\right. \\
& =s_{y y}-\frac{s_{x y}^{2}}{s_{x x}}+n(\alpha-a)^{2}+s_{x x}\left(\beta-\frac{s_{x y}}{s_{x x}}\right)^{2} \\
& \therefore \Sigma\left[\mathrm{Y}_{i}-\alpha-\beta \mathrm{x}_{i}\right]^{2}=\mathrm{s}^{2}+\mathrm{n}(\alpha-\mathrm{a})^{2}+\mathrm{S}_{\mathrm{xx}}(\beta-b)^{2} \text {. } \\
& \text { Logo: } \\
& \begin{aligned}
p\left(y ; \alpha ; \beta ; \sigma^{2}\right)= & k_{4} \cdot(2 \pi)^{-n / 2} \cdot\left(\sigma^{2}\right)^{-1 / 2(n+2)} \cdot e^{-\left(S^{2} / 2 \sigma^{2}\right)} \cdot e^{-\left(n / 2 \sigma^{2}\right)(\alpha-a)^{2}} x \\
& x e^{-\left(S x x / 2 \sigma^{2}\right)(\beta-b)^{2}}
\end{aligned}
\end{aligned}
$$


então:

$$
\begin{aligned}
& k^{-1}=p(y)=k_{4}(2 \pi)^{-n / 2} \int_{0}^{\infty}\left(\sigma^{2}\right)^{-1 / 2(n+2)} \cdot e^{-\left(S^{2} / 2 \sigma^{2}\right)}\left\{\int_{-\infty}^{\infty} e^{-\left(n / 2 \sigma^{2}\right)(\alpha-a)^{2}}\left[\int_{-\infty}^{\infty} e^{\left.-(s) / 2 \sigma^{2}\right)(\beta-b)^{2}} d B\right] d \alpha\right\} \sigma^{2} \\
& \text { Resolvendo as integrais, obtemos: } \\
& k=\frac{1}{k_{4}} \cdot \frac{\sqrt{n} \sqrt{S_{x x}}}{\Gamma\left(\frac{n}{2}-1\right)}(\pi)^{1 / 2(n-2)} s^{n-2} \\
& \pi\left(\alpha ; \beta ; \sigma^{2} \mid y\right)=k \cdot \pi\left(\alpha ; B ; \sigma^{2}\right) \cdot p\left(y \mid \alpha ; \beta ; \sigma^{2}\right) \\
& =\frac{1}{k_{4}} \cdot \frac{\sqrt{n} \sqrt{s}}{\Gamma\left(\frac{n}{2}-1\right)}(\pi)^{1 / 2(n-2)} \cdot s^{n-2} \cdot k_{4}(2 \pi)^{-n / 2}\left(\sigma^{2}\right)^{-1 / 2(n+2)} \cdot \exp \left\{-\frac{1}{2 \sigma^{2}}\left[s^{2}+n(\alpha-a)^{2}+s_{x x}(e-b)^{2}\right]\right\} \\
& \pi\left(\alpha ; \beta ; \sigma^{2} \mid y\right)=\frac{\sqrt{n} \sqrt{s} x}{2^{n / 2} \cdot \pi} \cdot \frac{s^{n-2}}{\Gamma\left(\frac{n}{2}-1\right)}\left(\sigma^{2}\right)^{-1 / 2(n+2)} \cdot \exp \left\{-\frac{1}{2 \sigma^{2}}\left[s^{2}+n(\alpha-a)^{2}+s_{x x}(\beta-b)^{2}\right]\right\}
\end{aligned}
$$

\subsubsection{1 - F.D.P.s marginais "a posteriori" para os parâmetros}

a) F.D.P. marginal "a posteriori" para B

Integrando (3.11) em relação a $\alpha$, obtemos:

$$
\pi\left(\beta ; \sigma^{2} \mid y\right)=A \int_{-\infty}^{\infty} e^{-\left(n / 2 \sigma^{2}\right)(\alpha-a)^{2}} d \alpha=A \frac{\sqrt{2 \pi}}{\sqrt{n}}\left(\sigma^{2}\right)^{1 / 2}
$$


onde A é tudo que não envolve $\underline{\alpha}$ em (3.11)

$\pi\left(\beta ; \sigma^{2} \mid y\right)=\frac{\sqrt{S_{x x}} S^{n-2}}{2^{1 / 2(n-1)} \sqrt{\pi} \Gamma\left(\frac{n}{2}-1\right)}\left(\sigma^{2}\right)^{-1 / 2(n+1)} \cdot e^{-\left(1 / 2 \sigma^{2}\right)\left[S^{2}+S_{x x}(\beta-b)^{2}\right]}$

Integrando em relação a $\sigma^{2}$, encontramos:

$$
\begin{aligned}
& \pi(\beta \mid y)=\frac{\sqrt{S_{x x}} s^{n-2}}{2^{1 / 2(n-1)} \sqrt{\pi} \Gamma\left(\frac{n}{2}-1\right)} \int_{0}^{\infty}\left(\sigma^{2}\right)^{-1 / 2(n+1)} \cdot e^{-\left(1 / 2 \sigma^{2}\right)\left[s^{2}+S_{x x}(\beta-b)^{2}\right]} d \sigma^{2} \\
& \therefore \pi(\beta \mid y)=\frac{\sqrt{S} s^{n-2}}{B\left(\frac{n}{2}-1 ; \frac{1}{2}\right)}\left[s^{2}+S_{x x}(\beta-b)^{2}\right]^{-1 / 2(n-1)} \\
& \text { b) F.D.P. marginal "a posteriori" para } \alpha
\end{aligned}
$$

Integrando (3.11), em relação a $\beta$ e, em seguida, em re1ação a $\sigma^{2}$, teremos:

$$
\pi\left(\alpha ; \sigma^{2} \mid y\right)=\frac{\sqrt{n} s^{n-2}\left(\sigma^{2}\right)^{-1 / 2(n+1)}}{2^{1 / 2(n-1)} \sqrt{\pi} \cdot \Gamma\left(\frac{n}{2}-1\right)} \cdot e^{-\left(1 / 2 \sigma^{2}\right)\left[s^{2}+n(\alpha-a)^{2}\right]}
$$

e

$$
\pi(\alpha \mid y)=\frac{\sqrt{n} s^{n-2}}{B\left(\frac{n}{2}-1 ; \frac{1}{2}\right)}\left[s^{2}+n(\alpha-a)^{2}\right]^{-1 / 2(n-1)}
$$

C) F.D.P. marginal "a posteriori" para $\sigma^{2}$

Integrando (3.12) em relação a $\beta$ ou integrando (3.14) em relação a $\alpha$ encontraremos : 
63.

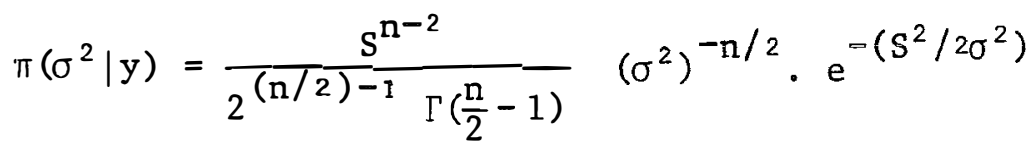

e) F.D.P. conjunta "a posteriori" para $\alpha$ e $\beta$

$$
\text { Integrando (3.11) em relação a } \sigma^{2} \text {, obteremos: }
$$

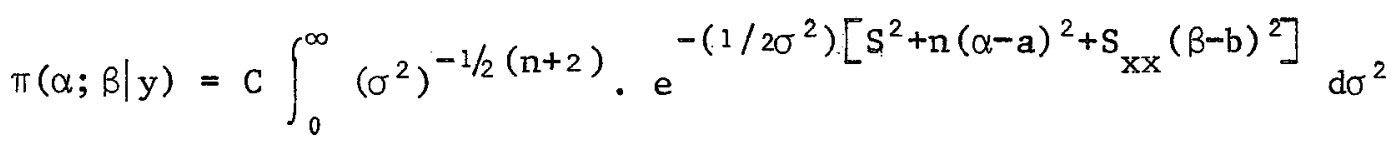

onde $C$ inclui todos os termos que não envolvem $\sigma^{2}$.

$$
\therefore \pi(\alpha ; \beta \mid y)=\frac{\sqrt{n} \sqrt{S_{x x}}\left(S^{2}\right)^{(n / 2)-1}}{\pi B\left(\frac{n}{2}-1 ; 1\right)}\left[s^{2}+n(\alpha-a)^{2}+S_{x x}(\beta-b)^{2}\right]^{-n / 2}
$$

\subsubsection{2 - Estimadores de Bayes para os parāmetros}

Como vimos em 3.1.2.2, o estimador de Bayes deve minimizar o Risco "a posteriori". Se utilizarmos a função Perda Quadrātica, o estimador de Bayes é a média da distribuição "a posterio ri".

a) Estimador para $\alpha$

Na f.d.p. "a posteriori" de $\alpha$ em (3.15) fazemos $v=$ $=\mathrm{n}-2$ e determinamos a esperança matemātica:

$$
\pi(\alpha \mid y)=\frac{\sqrt{n} s^{v}}{B\left(\frac{v}{2} ; \frac{1}{2}\right)}\left[s^{2}+n(\alpha-a)^{2}\right]^{-1 / 2(v+1)}
$$




$$
\begin{aligned}
\alpha^{*}=E(\alpha) & =\int_{-\infty}^{\infty} \alpha \cdot \pi(\alpha \mid y) d \alpha=\frac{\sqrt{n} s^{v}}{B\left(\frac{v}{2} ; \frac{1}{2}\right)} \int_{-\infty}^{\infty} \alpha \cdot\left[s^{2}+n(\alpha-a)^{2}\right]^{-1 / 2(v+1)} d \alpha \\
& =\frac{\sqrt{n} s^{v}}{B\left(\frac{v}{2} ; \frac{1}{2}\right)} \int_{-\infty}^{\infty} \alpha s^{-(v+1)}\left[1+\left(\frac{\alpha-a}{s / \sqrt{n}}\right)^{2}\right]^{-1 / 2(v+1)} d \alpha
\end{aligned}
$$$$
\text { fazendo } \frac{\alpha-a}{S / \sqrt{n}}=w \Longrightarrow \alpha=\frac{S}{\sqrt{n}} w+a \Longrightarrow d \alpha=\frac{S}{\sqrt{n}} d w
$$$$
\alpha^{*}=\frac{\sqrt{\mathrm{n}} \mathrm{S}^{-1}}{B\left(\frac{\mathrm{v}}{2} ; \frac{1}{2}\right)} \cdot \frac{\mathrm{S}}{\sqrt{\mathrm{n}}} \int_{-\infty}^{\infty}\left(a+\frac{\mathrm{S}}{\sqrt{\mathrm{n}}} \mathrm{w}\right)\left(1+\mathrm{w}^{2}\right)^{-1 / 2(v+1)} \mathrm{dw}
$$$$
\alpha *=\frac{1}{B\left(\frac{v}{2} ; \frac{1}{2}\right)}\left[a \cdot \int_{-\infty}^{\infty}\left(1+w^{2}\right)^{-1 / 2(v+1)} d w+\frac{S}{\sqrt{n}} \int_{-\infty}^{\infty} w\left(1+w^{2}\right)^{-1 / 2(v+1)} d w\right]
$$

A primeira integral dentro dos colchetes vale $B\left(\frac{V}{2} ; \frac{1}{2}\right)$.

e a segunda integral vale zero, logo:

$$
\begin{aligned}
& \alpha^{*}=\frac{1}{B\left(\frac{v}{2} ; \frac{1}{2}\right)} \cdot a \cdot B\left(\frac{v}{2} ; \frac{1}{2}\right) \\
\therefore \quad & \alpha^{*}=a=\bar{Y}=\frac{1}{n} \sum^{n} Y_{i}
\end{aligned}
$$

Este resultado era o esperado, uma vez que, sem infor mação "a priori", o peso da informação sobre a próvém do resultado observado na amostra.

b) Estimador para $B$

De maneira anāloga ao que foi feito anteriormente para $\alpha$, teremos: 
65 .

$$
\beta *=E(\beta)=\int_{-\infty}^{\infty} \beta \cdot \pi(\beta \mid y) d \beta=\frac{\sqrt{S} s^{v}}{B\left(\frac{v}{2} ; \frac{1}{2}\right)} \int_{-\infty}^{\infty} \beta\left[S^{2}+S_{x x}(\beta-b)^{2}\right]^{-1 / 2(v+1)} d \beta
$$

e encontraremos:

$$
\beta^{*}=b=\frac{S_{x y}}{S_{x x}}=\frac{\sum x_{i} y_{i}}{\sum x_{i}^{2}}
$$

Vale aqui a mesma observação feita para o resultado $(3.18)$.

\subsubsection{3 - Testes de hipōteses a respeito dos parā- metros}

a) Considerando os termos que não incluem os parâmetros $\alpha$ e $\beta$ como constantes, podemos escrever (3.11) na forma:

$$
\begin{aligned}
& \pi(\alpha ; \beta \mid y) \propto e^{-\left(n / 2 \sigma^{2}\right)(\alpha-a)^{2}} e^{-\left(S_{x x} / 2 \sigma^{2}\right)(\beta-b)^{2}} \\
& \text { Isto nos mostra que } \alpha \text { e } \beta \text { (independentes) têm as se- }
\end{aligned}
$$
guintes distribuições "a posteriori", respectivamente:

$$
\begin{aligned}
& \alpha \cap N\left(a ; \frac{\sigma^{2}}{n}\right) \\
& \beta \cap N\left(b ; \frac{\sigma^{2}}{s_{x x}}\right)
\end{aligned}
$$

As distribuições indicadas em (3.20) concordam com os resultados observados em (3.18) e (3.19) para os estimadores de Bayes para os parâmetros $\alpha$ e $\beta$. Por outro lado, nos mostram também 
66.

que as variâncias dessas distribuições são:

$$
\mathrm{V}(\alpha)=\frac{\sigma^{2}}{\mathrm{n}}
$$

e

$$
V(\beta)=\frac{\sigma^{2}}{S_{x x}}
$$

Para as afirmações probabilỉsticas "a posteriori", pa ra os intervalos de confiança e para testes de significância acerca dos parâmetros, podemos considerar as seguintes variáveis com dis tribuições normais padronizadas:

$$
\begin{aligned}
& \frac{\alpha-a}{\sqrt{V(\alpha)}}=\frac{\alpha-a}{\left(\sigma^{2} / n\right)^{1 / 2}} \cap N(0 ; 1) \\
& \frac{\beta-b}{\sqrt{V(\beta)}}=\frac{\beta-b}{\left(\sigma^{2} / s_{x x}\right)^{1 / 2}} \cap N(0 ; 1)
\end{aligned}
$$

Como a variância é desconhecida, podemos substituí-1a por uma estimativa e as variáveis terão distribuição " $t$ " como mostramos a seguir.

Por (3.15) escrevemos:

$$
\pi(\alpha \mid y) \propto\left[s^{2}+n(\alpha-a)^{2}\right]^{-1 / 2(n-1)}
$$

fazendo: $v=n-2$ e $s^{2}=\frac{s^{2}}{v}$, teremos:

$$
\pi(\alpha \mid y) \propto\left[v s^{2}+n(\alpha-a)^{2}\right]^{-1 / 2(v+1)} .
$$


67 .

Cons ideremos

$$
t_{1}=\frac{\alpha-a}{\left[s^{2} / n(n-2)\right]^{1 / 2}}=\frac{\alpha-a}{\left[s^{2} / n\right]^{1 / 2}},
$$

onde $s^{2} / \mathrm{n}$ é a estimativa da variância de $\alpha: \bar{v}(\alpha)=s^{2} / n$.

o Jacobiano da transformação é constante,e podemos es crever:

$$
\begin{aligned}
& \pi\left(t_{1} \mid y\right) \propto\left[1+\frac{t_{1}^{2}}{v}\right]^{-1 / 2(v+1)} \\
& \pi\left(t_{1} \mid y\right)=\frac{1}{\sqrt{v} B\left(\frac{v}{2} ; \frac{1}{2}\right)}\left(1+\frac{t_{1}^{2}}{v}\right)^{-1 / 2(v+1)}
\end{aligned}
$$

Esta ūltima expressão da f.d.p. "a posteriori" nos mostra que a variāvel aleatória

$$
t_{1}=\frac{\alpha-a}{\left(s^{2} / n v\right)^{1 / 2}}=\frac{\alpha-a}{\sqrt{v}(\alpha)}
$$

tem distribuição de " $t$ " com $v=n-2$ graus de liberdade.

De maneira análoga, podemos verificar que, de (3.13)

$$
\begin{gathered}
\pi(\beta \mid y) \propto\left[s^{2}+s_{x x}(\beta-b)^{2}\right]^{-1 / 2(v+1)} \\
\pi\left(t_{2} \mid y\right)=\frac{1}{\sqrt{v} \cdot B\left(\frac{v}{2} ; \frac{1}{2}\right)}\left(1+\frac{t_{2}^{2}}{v}\right)^{-1 / 2(v+1)} \\
\text { Concluímos que } t_{2}=\frac{\beta-b}{\left[s^{2} / v\left(s_{x x}\right)\right]^{1 / 2}}=\frac{\beta-b}{\sqrt{v}(\beta)} \text { tem dis- } \\
\text { tribuição de " } t \text { " com } v=n-2 \text { graus de } 1 \text { iberdade. }
\end{gathered}
$$


68.

Assim, os valores de $t_{1}$ e $t_{2}$, acima descritos, são utilizados para testar hipóteses sobre valores dos parâmetros (considerando-se cada um individualmente), sendo comparados com valores de tabelas de " $t$ " para $v=n-2$ graus de 1 iberdade, como no processo usual.

b) De (3.16) podemos escrever:

$$
\pi\left(\sigma^{2} \mid y\right) \propto\left(\sigma^{2}\right)^{-n / 2} \cdot e^{-\left(S^{2} / 2 \sigma^{2}\right)}
$$

Fazendo $w=\frac{S^{2}}{\sigma^{2}}, o$ jacobiano da transformação vale $-\frac{\left(\sigma^{2}\right)^{2}}{s^{2}}$, então:

$$
\begin{aligned}
& \pi(w \mid y) \propto\left(\frac{s^{2}}{w}\right)^{-n / 2} \cdot e^{-w / 2} \cdot \frac{s^{2}}{w^{2}} \\
& \pi(w \mid y) \propto w^{(v / 2)-1} \cdot e^{-w / 2}
\end{aligned}
$$

ou

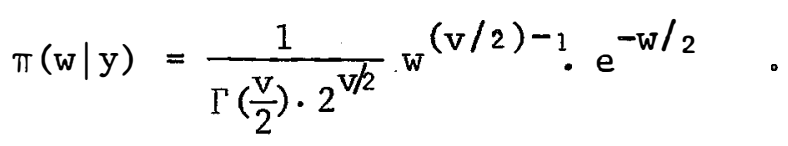

Esta ültima expressão nos mostra que a variāve1 $\mathrm{w}=\frac{\mathrm{S}^{2}}{\sigma^{2}}$ tem distribuição de "qui-quadrado" com $\mathrm{v}=\mathrm{n}-2$ graus de $1 \underline{\mathrm{i}}$ berdade.

c) Para testar hipóteses conjuntas sobre $\alpha$ : e $\beta$, consideremos (3.17), que pode ser escrita

$$
\pi(\alpha, \beta \mid y) \propto\left[s^{2}+n(\alpha-a)^{2}+s_{x x}(\beta-b)^{2}\right]^{-n / 2}
$$


69.

$$
\begin{array}{r}
\pi(\alpha, \beta \mid y) \propto\left(s^{2}\right)^{-n / 2}\left[1+\frac{n(\alpha-a)^{2}+s_{x x}(\beta-b)^{2}}{s^{2}}\right]^{-n / 2} \\
\pi(\alpha, \beta \mid y) \propto\left[1+\frac{n(\alpha-a)^{2}+s_{x x}(\beta-b)^{2}}{v s^{2}}\right]^{-n / 2}
\end{array}
$$

sendo a constante de proporcionalidade igual a

$$
\frac{\sqrt{\mathrm{n}} \sqrt{\mathrm{S}}}{\mathrm{B}\left(\frac{\mathrm{n}-2}{2} ; 1\right)} \cdot \frac{1}{\mathrm{vs}^{2}}
$$

\section{Fazendo:}

$$
F=\frac{n(\alpha-a)^{2}+s_{x x}(\beta-b)^{2}}{2 s^{2}} \text {, }
$$

o Jacobiano da transformação vale $\frac{2 s^{2}}{\sqrt{n} \sqrt{S_{x x}}}$ e escrevemos:

$$
\begin{aligned}
& \pi(F \mid y)=\frac{\sqrt{n} \sqrt{S} x x}{B\left(\frac{n-2}{2} ; 1\right)} \cdot \frac{1}{v s^{2}}\left[1+\frac{2}{v} F\right]^{-n / 2} \cdot \frac{2 s^{2}}{\sqrt{n} \sqrt{S}} \\
& \pi(F \mid y)=\frac{1}{B\left(\frac{v}{2} ; 1\right)}\left(\frac{2}{v}\right)\left[1+\frac{2}{v} F\right]^{-1 / 2(v+2)}
\end{aligned}
$$

Comparando com a f.d.p. de uma distribuição de F com $v_{1}$ e $v_{2}$ graus de 1 iberdade:

$$
\mathrm{p}\left(\mathrm{F} \mid \mathrm{v}_{1} ; \mathrm{v}_{2}\right)=\frac{1}{\mathrm{~B}\left(\frac{\mathrm{v}}{2} ; \frac{\mathrm{v}_{2}}{2}\right)}\left(\frac{\mathrm{v}_{1}}{\mathrm{v}_{2}}\right)^{\mathrm{v}_{1} / 2} \cdot \mathrm{F}^{\left(\mathrm{v}_{1} / 2\right)-1}\left[1+\frac{\mathrm{v}_{1}}{\mathrm{v}_{2}}\right]^{-1 / 2\left(\mathrm{v}_{1}+\mathrm{v}_{2}\right)}
$$

notamos que a ültima expressão tem a mesma forma com $v_{1}=2$ e $v_{2}=v$. Logo: 


$$
F=\frac{n(\alpha-a)^{2}+s_{x x}(\beta-b)^{2}}{2 s^{2}}
$$

tem distribuição de $\mathrm{F}$ com 2 e $\mathrm{v}$ graus de liberdade.

$$
\text { Quando quisermos testar uma única hipótese envolven- }
$$
do, concomitantemente, os valores dos dois parâmetros $\alpha$ e $\beta$, utilizamos o teste F. Vale a pena notar que, neste caso, testar as hipóteses

$$
H_{0}: \alpha=\alpha_{1} \text { e } \beta=\beta_{1}
$$

contra

$$
\mathrm{H}_{\mathrm{A}}: \alpha \neq \alpha_{1} \text { e } \beta \neq \beta_{1}
$$

não significa fazer dois testes consecutivos para $H_{0}: \alpha=\alpha_{1}$ e, em seguida, outro para a hipóțese $\mathrm{H}_{0}: \beta=\beta_{1}$.

Este teste de hipótese conjunta sobre os parâmetros de $\alpha=\alpha_{1}$ e $\beta=\beta_{1}$, significa testar a hipótese de que a equação, de regressão tem equação: $y=\alpha_{1}+\beta_{1} x$.

d) 0 teste de significância para $\beta=0$, ou seja, para a independência entre as duas variáveis, pode ser colocado sob outra forma, comparável ao que se faz normalmente na anālise de variância.Pe 1o que foi visto anteriormente em (a), e pela simetria da distribui ção de "t", podemos elevar ao quadrado, obtendo

$$
t_{\varepsilon}^{2}=\frac{b^{2}}{s^{2} / v\left(s_{x x}\right)}=\frac{b^{2}}{\left[s_{y y}-\frac{s_{x y}^{2}}{s_{x x}}\right] /(n-2)}
$$


Escrevendo:

$$
S_{y y}=\left[S_{y y}-\frac{s_{x y}^{2}}{S_{x x}}\right]+\left[\frac{s_{x y}^{2}}{S_{x x}}\right]
$$

onde $\mathrm{S}_{\mathrm{yy}}=\sum^{\mathrm{n}} \mathrm{y}_{\mathrm{i}}^{2}=$ Soma de Quadrados Total;

$$
\begin{aligned}
& \frac{S_{x y}^{2}}{S_{x x}}=b . s_{x y}=\text { Soma de Quadrados devida à Regressão; } \\
& s_{y y}-\frac{S_{x y}^{2}}{S_{x x}}=\text { Soma de Quadrados Residual。 }
\end{aligned}
$$

Desta forma:

$$
\text { SQ Total }=S Q \operatorname{Reg}+\mathrm{SQ} \operatorname{Res}
$$

e, desde que $t_{\varepsilon}^{2}(v)$ e equivalente a $F_{1, v}$, podemos usar este $\vec{u} 1 t i m o$ teste

$$
t_{\varepsilon}^{2}=F_{1, v}=\frac{\frac{S Q R e g}{1}}{\frac{S Q \operatorname{Res}}{v}}=\frac{Q M \operatorname{Reg}}{Q M \operatorname{Res}} .
$$

Estes resultados concordam com aqueles usuais da anā 1ise de variância, e podemos fazer:

\begin{tabular}{lcccl}
\hline $\begin{array}{l}\text { Causas da } \\
\text { Variação }\end{array}$ & $\begin{array}{c}\text { Graus de } \\
\text { Liberdade }\end{array}$ & $\begin{array}{c}\text { Soma de } \\
\text { Quadrados }\end{array}$ & $\begin{array}{c}\text { Quadrado } \\
\text { Médio }\end{array}$ & $\mathrm{F}$ \\
\hline $\begin{array}{l}\text { Regressão } \\
\text { Resíduo }\end{array}$ & 1 & $\mathrm{~s}_{\mathrm{xy}}^{2} / \mathrm{s}_{\mathrm{xx}}=\mathrm{b}^{2} \cdot \sum \mathrm{x}_{\mathrm{i}}^{2}$ & $\mathrm{~b}^{2} \cdot \mathrm{s}_{\mathrm{xx}}$ & QMReg/QMRes \\
\hline Total & $\mathrm{v}=\mathrm{n}-2$ & $\mathrm{~s}_{\mathrm{yy}}-\left(\mathrm{s}_{\mathrm{xy}}^{2} / \mathrm{s}_{\mathrm{xx}}\right)=\mathrm{s}^{2}$ & $\mathrm{~s}^{2} / \mathrm{v}=\mathrm{s}^{2}$ & \\
& $\mathrm{n}-1$ & $\mathrm{~s}_{\mathrm{yy}}$ & &
\end{tabular}




\subsubsection{4 - Intervalos de confiança para os parāme- tros}

Sendo $t_{0} o$ valor crítico de tabela com $v=n-2$ graus de liberdade e a um nível de confiança estabelecido, os intervalos de confiança para $\alpha$ e $\beta$ são, respectivamente:

$$
a-t_{0} \cdot s(\alpha)<\alpha<a+t_{0} \cdot s(\alpha)
$$

e

$$
b-t_{0} \cdot s(\beta)<\beta<b+t_{0} \cdot s(\beta)
$$

onde $s(\alpha)=\sqrt{\hat{V}(\alpha)}$ e $s(\beta)=\sqrt{\hat{V}(\beta)}$.

Embora o método de Bayes forneça no caso em questão, os mesmos valores numéricos obtidos pelos métodos usuais, a sua interpretação do intervalo de confiança é diferente. Aqui, obtida a f.d.p. "a posteriori" para $\beta$, por exemplo, e sendo $R C \Omega$ (uma parti cular sub-região do espaço paramétrico) e 1- $\varepsilon$ o nível de confiança estabelecido para se encontrar $t_{0}$, o intervalo de confiança acima significa que:

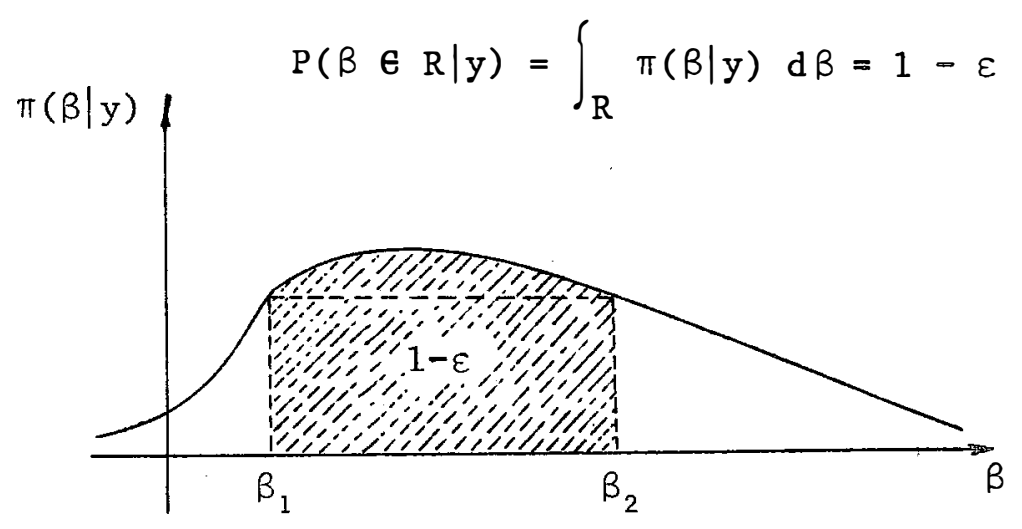

Figura 4 - Intervalo de confiança de Bayes $\left(\beta_{1} ; \beta_{2}\right)$ para o parâmetro $\beta$, ao nível de confiança $\varepsilon$. 
Notemos que este intervalo é certamente único apenas nas distribuições unimodais. Nestes casos, se a distribuição for si métrica, centrada no valor modal, o intervalo com dada probabilidade contendo $\beta$ é o intervalo bayesiano de máxima densidade "a posteriori". E claro que o interesse é obter o intervalo de menor amplitude possível. Logo, devemos minimizar $\left(\beta_{2}-\beta_{1}\right)$, sujeito à condição de que

$$
\int_{\beta_{1}}^{\beta_{2}} \pi(\beta \mid y) d \beta=1-\varepsilon
$$

Usando o método dos multiplicadores de Lagrange, podemos formar:

$$
\phi=\beta_{2}-\beta_{1}+\lambda\left[\int_{\beta_{1}}^{\beta_{2}} \pi(\beta \mid y) d \beta-1+\varepsilon\right]
$$

Derivvando parcialmente em relação a $\beta_{1}$ e $\beta_{2}$ e igualando a zero:

$$
\begin{aligned}
& \frac{\partial \phi}{\partial \beta_{1}}=-1-\lambda \pi\left(\beta_{1} \mid y\right)=0 \Longrightarrow 1+\lambda \pi\left(\beta_{1} \mid y\right)=0 \\
& \frac{\partial \phi}{\partial \beta_{2}}=+1+\lambda \pi\left(\beta_{2} \mid y\right)=0 \Longrightarrow 1+\lambda \pi\left(\beta_{2} \mid y\right)=0
\end{aligned}
$$

donde $\pi\left(\beta_{1} \mid y\right)=\pi\left(\beta_{2} \mid y\right)$.

$$
\text { Assim, determinando } \beta_{1} \text { e } \beta_{2} \text { tal que } \pi\left(\beta_{1} \mid y\right)=\pi\left(\beta_{2} \mid y\right) \text {, }
$$

o intervalo $\left[\beta_{1} ; \beta_{2}\right]$ será minimo nos casos de distribuições unimodais (como ilustra a Figura 4). 
Na teoria não-bayesiana, o intervalo de confiança é tomado como aleatório e tendo a probabilịdade $(1-\varepsilon)$ de cobrir o ver dadeiro valor do parâmetro. Em outras palavras, se considerarmos um grande número de amostras, os intervalos considerados incluirão o parâmetro em $100(1-\varepsilon) \%$ dos casos.

$\mathrm{Na}$ teoria bayesiana, onde as afirmações são condicio nais às observações amostrais, com as quais se estabelece o interva 1o, a interpretação é de que a probabilidade "a poster iori" de que o verdadeiro valor de $B$ esteja no intervalo é de $100(1-\varepsilon) \%$.

\subsubsection{5 - Intervalo de previsão}

Em algumas circunstâncias é necessārio estimar o valor de $\mathrm{Y}$ que corresponda a um valor de $\mathrm{X}$ que não existe na amostra; estabelecer limites para o valor esperado de $\mathrm{Y}$ para um dado $\mathrm{X}_{\mathrm{O}}$, ou mesmo testar a hipótese de que a reta de regressão passe através.do ponto $\left(\mathrm{x}_{\mathrm{o}} ; \mathrm{y}_{\mathrm{o}}\right)$.

Nestes casos devemos utilizar a distribuição "a posteriori" de

$$
\delta=E\left(y \mid x_{0}\right)=\alpha+\beta\left(x_{0}-\bar{x}\right) \quad .
$$

Para uma dada variância, as expressões (3.11) e (3.2 0)

nos mostram que $\mathrm{E}\left(\mathrm{y} \mid \mathrm{x}_{\mathrm{o}}\right)$ tem distribuição normal com:

$$
\text { média }=E\left[E\left(y \mid x_{0}\right)\right]=a+b\left(x_{0}-\bar{x}\right)=\hat{\delta}
$$


75.

$$
\begin{aligned}
\text { variância } & =V\left[E\left(y \mid x_{0}\right)\right]=V\left[\alpha+\beta x_{0}\right]=V(\alpha)+x_{0}^{2} V(\beta) \\
& =\frac{\sigma^{2}}{n}+x_{0}^{2} \cdot \frac{\sigma^{2}}{S_{x x}}=\left(\frac{1}{n}+\frac{x_{0}^{2}}{S_{x x}}\right) \cdot \sigma^{2}
\end{aligned}
$$

Logo:

$$
\delta=E\left[y \mid x_{0}\right] \cap N\left\{a+b x_{0} ;\left(\frac{1}{n}+\frac{x_{0}^{2}}{s_{x x}}\right) \cdot \sigma^{2}\right\}
$$

Também, para um dado $x_{0}$, a distribuição conjunta "a posteriori" de $\delta$ e $\sigma^{2}$ será:

$$
\begin{aligned}
& \pi\left(\delta ; \sigma^{2} \mid \mathrm{x}_{0}\right)=\pi\left(\delta \mid \sigma^{2} ; \mathrm{x}_{\mathrm{o}}\right) \cdot \pi\left(\sigma^{2} \mid \mathrm{x}_{\mathrm{o}}\right) \\
& \propto\left(\sigma^{2}\right)^{-1 / 2} \cdot e^{-\left(1 / 2 \sigma^{2}\right)\left[(1 / n)+\left(x_{0}^{2} / S_{x x}\right)\right]^{-1}[\delta-E(\delta)]^{2}} x \\
& x\left(\sigma^{2}\right)^{-(1 / 2 v)-1} \cdot e^{-S^{2} / 2 \sigma^{2}} \\
& \left.\left.\left.\propto\left(\sigma^{2}\right)^{-1 / 2(v+3)} \cdot e^{-\left(1 / 2 \sigma^{2}\right)\left\{S^{2}+\left[(1 / n)+\left(x_{o}^{2} / S\right.\right.\right.} x_{x x}\right)\right]^{-1}[\delta-E(\delta)]^{2}\right\} \\
& \text { Integrando em relação a } \sigma^{2} \text { : } \\
& \pi\left(\delta \mid \mathrm{x}_{\mathrm{o}}\right) \propto\left\{\mathrm{S}^{2}+\left(\frac{1}{\mathrm{n}}+\frac{\mathrm{x}_{\mathrm{o}}^{2}}{\mathrm{~S}_{\mathrm{xx}}}\right)^{-1}\left[\delta-\mathrm{E}(\delta)^{2}\right]\right\}-1 / 2(\mathrm{v}+1) \\
& \pi\left(\delta \mid x_{0}\right) \propto\left\{1+\frac{[\delta-E(\delta)]^{2}}{S^{2}\left(\frac{1}{n}+\frac{x_{0}^{2}}{S_{x x}}\right)}\right\}^{-1 / 2(v+1)}
\end{aligned}
$$


76.

Se fizermos:

$$
t_{3}=\frac{\delta-E(\delta)}{\left[S^{2}\left(\frac{1}{n}+\frac{x_{0}^{2}}{S_{x x}}\right) / v\right]^{1 / 2}}
$$

o Jacobiano da transformação é constante e

$$
\pi\left(t_{3} \mid x_{0}\right) \propto\left\{1+\frac{t_{3}^{2}}{v}\right\}^{-1 / 2(v+1)}
$$

o que mostra que a distribuição "a posteriori" de $E\left(y ! x_{0}\right)$ é tal que

$$
\frac{E\left(y \mid x_{0}\right)-\left(a+b x_{0}\right)}{\left[\left(\frac{1}{n}+\frac{x_{0}}{s_{x x}}\right) s^{2}\right]^{1 / 2}}
$$

tem distribuição de " $t$ " com $v=n-2$ graus de 1 iberdade.

Esta ültima conclusão nos permite utilizar o teste " $t$ " para testar a hipótese de que $E\left(y \mid x_{0}\right)=y_{0}$, ou seja, que a reta de regressão passa pelo ponto $\left(x_{0} ; y_{0}\right)$. Além disso podemos estabelecer o intervalo de confiança para $E\left(y \mid x_{0}\right)$, considerando $t_{0}$ como valor critico de " $t$ " com (n-2) graus de liberdade, e ao nivel de confiança estabelecido e encontraremos:

$$
\delta-t_{0} \cdot s\left(y_{0}\right)<E\left(y \mid x_{0}\right)<\delta+t_{0} \cdot s\left(y_{0}\right)
$$

onde

$$
\delta=E\left[E\left(y \mid x_{0}\right)\right] \text { e } s^{2}\left(y_{0}\right)=\left(\frac{1}{n}+\frac{x_{0}^{2}}{s_{x x}}\right) \cdot \frac{s^{2}}{v}
$$


Em outras situaçōes, queremos prever o valor da variāvel dependente para $x=x_{0}$. A precisão desta previsão é feita pe lo intervalo de previsão. Para um determinado valor de $x=x_{0}, o s$ va lores de $Y$ (como variável aleatória) variam em torno de $E\left(y \mid x_{0}\right)$ (sua verdadeira média), com variância $\sigma^{2}$. Como $y_{0}$, estimativa de $E\left(y \mid x_{0}\right)$, também é varriável aleatória, a variância $\sigma_{p}^{2}$ é dada por:

$$
\begin{aligned}
& \sigma_{p}^{2}=\sigma^{2}+V\left[E\left(y \mid x_{0}\right)\right]=\sigma^{2}+\left(\frac{1}{n}+\frac{x_{0}^{2}}{S_{i x}}\right) \sigma^{2} \\
& \sigma_{p}^{2}=\left(1+\frac{1}{n}+\frac{x_{0}^{2}}{S_{x x}}\right) \cdot \sigma^{2}
\end{aligned}
$$

o intervalo de previsão para uma nova observação será estimado por:

$$
\begin{aligned}
& \hat{\delta}-t_{o} \cdot s_{p}<y_{0}<\hat{\delta}+t_{o} \cdot s_{p} \\
& \text { onde } s_{p}^{2}=\left(1+\frac{1}{n}+\frac{x_{0}^{2}}{s_{x x}}\right) \cdot s^{2} \text {. } \\
& \text { 3.3.3 - Uso da F.D.P. "a priori" informativa } \\
& \text { 3.3.3.1 - F.D.P. "a priori" normal para os coefi- } \\
& \text { cientes e variāncia residual conhecida }
\end{aligned}
$$


78 .

va "a posteriori". O pouco interesse prático desta situação è que consideramos a variância conhecida, o que normalmente não ocorre.

O fato de considerarmos a distribuição "a priori" nor mal para os parâmetros significa que as experiências anteriores nos levaram a ter um grau de confiança maior num determinado valor para o parâmetro, com certa variação, como vimos em (2.4). Desta forma, para $\alpha$ e $\beta$, consideramos as f.d.p. "a priori":

$$
\begin{aligned}
& \alpha \cap N\left(\alpha_{0} ; \sigma_{a}^{2}\right) \rightarrow \pi(\alpha)=\frac{1}{\sqrt{2 \pi \sigma_{a}^{2}}} e^{-\left(1 / 2 \sigma_{a}^{2}\right)\left(\alpha-\alpha_{0}\right)^{2}}, \\
& \beta \cap N\left(\beta_{0} ;\left(\sigma_{b}^{2}\right) \rightarrow \pi(\beta)=\frac{1}{\sqrt{2 \pi \sigma_{b}^{2}}} e^{-\left(1 / 2 \sigma_{b}^{2}\right)\left(\beta-\beta_{0}\right)^{2}},\right.
\end{aligned}
$$

e com variância conhecida $\rightarrow \sigma^{2}=\sigma_{0}^{2}$.

Sendo $\alpha$ e $\beta$ independentemente distribuídos, a distri buição conjunta "a priori" fica:

$$
T(\alpha ; \beta)=\frac{1}{2 \pi \cdot \sigma_{a} \cdot \sigma_{b}} e^{-\left(1 / 2 \sigma_{a}^{2}\right)\left(\alpha-\alpha_{0}\right)^{2}} \cdot e^{-\left(1 / 2 \sigma_{b}^{2}\right)\left(\beta-\beta_{o}\right)^{2}}
$$

Pelas pressuposições do modelo apresentado em (3.2), os $\mathrm{Y}_{i}$ serãc normal e independentemente distribuídos com média

$$
E\left[Y_{i} \mid \alpha, \beta, X_{i} ; \sigma_{\sigma}^{2}\right]=\alpha+\beta x_{i}
$$

e variância

$$
V\left(Y_{i} \mid x_{i} ; \beta ; \alpha\right)=\sigma_{0}^{2}
$$

A função de verossimilhança será dada por: 


$$
p\left(y \mid \alpha ; \beta ; \sigma_{0}^{2}\right)=(2 \pi)^{-n / 2} \cdot\left(\sigma_{0}^{2}\right)^{-n / 2} \cdot e^{-\left(1 / 2 \sigma_{0}^{2}\right) \sum\left(Y_{i}-\alpha-\beta x_{i}\right)^{2}}
$$

Para encontrarmos a f.d.p. da distribuição "a posteriori"

$$
\pi\left(\alpha ; \beta \mid y ; \sigma_{0}^{2}\right)=\frac{\pi(\alpha ; \beta) \cdot p\left(y \mid \alpha, \beta, \sigma_{0}^{2}\right)}{p(y)}
$$

devemos encontrar $p(y)$. Para isto, escrevemos a distribuição conjun ta:

$$
\begin{aligned}
& p(y ; \alpha ; \beta)=\frac{1}{2 \pi \sigma_{a} \cdot \sigma_{b}} \cdot e^{-\left(1 / 2 \sigma_{a}^{2}\right)\left(\alpha-\alpha_{0}\right)^{2}} \cdot e^{-\left(1 / 2 \sigma_{b}^{2}\right)\left(\beta-\beta_{0}\right)^{2}} \cdot(2 \pi)^{-n / 2} \times \\
& \times\left(\sigma_{0}^{2}\right)^{-n / 2} \cdot e^{-\left(1 / 2 \sigma_{0}^{2}\right)\left[S^{2}+n(\alpha-a)^{2}+S_{x x}(\beta-b)^{2}\right]} \\
& p(y ; \alpha ; \beta)=\frac{(2 \pi)^{-1 / 2(n+2)}}{\sigma_{a} \cdot \sigma_{b}}\left(\sigma_{0}^{2}\right)^{-n / 2} \cdot e^{-\left(S^{2} / 2 \sigma_{0}^{2}\right)} \times \\
& \times e^{-1 / 2\left[\left(S_{x x} / \sigma_{0}^{2}\right)(\beta-b)^{2}+\left(1 / \sigma_{b}^{2}\right)\left(\beta-\beta_{0}\right)^{2}\right]} \times \\
& \times e^{-1 / 2\left[\left(\pi / \sigma_{0}^{2}\right)(\alpha-a)^{2}+\left(1 / \sigma_{a}^{2}\right)\left(\alpha-\alpha_{0}\right)^{2}\right]}
\end{aligned}
$$

\section{Melhorando os expoentes, fazemos:}

$$
\begin{aligned}
& \frac{n}{\sigma_{0}^{2}} \alpha^{2}-\frac{2 \pi}{\sigma_{0}^{2}} a \alpha+\frac{n}{\sigma_{0}^{2}} a^{2}+\frac{1}{\sigma_{a}^{2}} \alpha^{2}-2 \frac{1}{\sigma_{a}^{2}} \alpha_{0} \alpha+\frac{1}{\sigma_{a}^{2}} \alpha_{0}^{2}= \\
& \quad=\left(\frac{n}{\sigma_{0}^{2}}+\frac{1}{\sigma_{a}^{2}}\right) \cdot \alpha^{2}-2\left(\frac{n}{\sigma_{0}^{2}} a+\frac{1}{\sigma_{0}^{2}} \alpha_{0}\right) \cdot \alpha+\frac{n}{\sigma_{0}^{2}} a^{2}+\frac{1}{\sigma_{0}^{2}} \alpha_{0}^{2}
\end{aligned}
$$


80 .

e chamamos:

$$
\begin{array}{lll}
M=\frac{\mathrm{n}}{\sigma_{0}^{2}}+\frac{1}{\sigma_{\mathrm{a}}^{2}} & \text { e } & \mathrm{N}=\frac{\mathrm{n}}{\sigma_{0}^{2}} \mathrm{a}+\frac{\mathrm{a}_{\mathrm{o}}}{\sigma_{\mathrm{a}}^{2}} \\
\mathrm{P}=\frac{\mathrm{S}_{\mathrm{xx}}}{\sigma_{0}^{2}}+\frac{1}{\sigma_{\mathrm{b}}^{2}} & \text { e } & \mathrm{Q}=\frac{\mathrm{b} \cdot \mathrm{S}_{\mathrm{xx}}}{\sigma_{0}^{2}}+\frac{\beta_{\mathrm{o}}}{\sigma_{\mathrm{b}}^{2}}
\end{array}
$$

Completando o quadrado da expressão acima:

$$
\left(\sqrt{\mathrm{M}} \alpha-\frac{\mathrm{N}}{\sqrt{\mathrm{M}}}\right)^{2}+\frac{\mathrm{na}^{2}}{\sigma_{\mathrm{O}}^{2}}+\frac{\alpha_{\mathrm{O}}^{2}}{\sigma_{\mathrm{a}}^{2}}-\frac{\mathrm{N}^{2}}{\mathrm{M}}=\left(\sqrt{\mathrm{M}} \alpha-\frac{\mathrm{N}}{\sqrt{\mathrm{M}}}\right)^{2}+\mathrm{R}=\mathrm{M}\left(\alpha-\frac{\mathrm{N}}{\mathrm{M}}\right)^{2}+\mathrm{R}
$$

De maneira semelhante o outro expoente fica:

$$
\frac{S_{x x}}{\sigma_{0}^{2}}(\beta-b)^{2}+\frac{1}{\sigma_{b}^{2}}\left(\beta-\beta_{o}\right)^{2}=P\left(\beta-\frac{Q}{P}\right)^{2}+T
$$

onde $T=\frac{b^{2} \cdot S_{x x}}{\sigma_{0}^{2}}+\frac{\beta_{0}^{2}}{\sigma_{b}^{2}}-\frac{Q^{2}}{P}$.

Logo:

$$
\begin{aligned}
& p(y ; \alpha ; \beta)=A \cdot e^{-1 / 2(R+T)} \cdot e^{-P / 2[\beta-(Q / P)]^{2}} \cdot e^{-M / 2[\alpha-(N / M)]^{2}} \\
& p(y)=A \cdot e^{-1 / 2(R+T)} \cdot \int_{-\infty}^{\infty} e^{-P / 2[\beta-(Q / P)]^{2}}\left[\int_{-\infty}^{\infty} e^{-M / 2[\alpha-(N / M)]^{2}} d \alpha\right] d \beta
\end{aligned}
$$

Resolvendo as integrais encontramos:

$$
p(y)=A \cdot e^{-1 / 2(R+T)} \cdot \frac{2 \pi}{\sqrt{\mathrm{P}} \cdot \sqrt{\mathrm{M}}}
$$


81.

Assim, a f.d.p. "a posteriori" ficarā:

$$
\begin{aligned}
& \pi\left(\alpha ; \beta \mid y ; \sigma_{0}^{2}\right)=\frac{A \cdot e^{-M / 2[\alpha-(N / M)]^{2}} \cdot e^{-P / 2[\beta-(Q / P)]^{2}} \cdot e^{-1 / 2(R+T)}}{A \cdot e^{-1 / 2(R+T)} \cdot \frac{2 \pi}{\sqrt{P} \cdot \sqrt{M}}} \\
& \therefore \quad \pi\left(\alpha ; \beta \mid y ; \sigma_{0}^{2}\right)=\frac{\sqrt{\mathrm{P}}}{\sqrt{2 \pi}} \cdot e^{-P / 2[\beta-(Q / P)]^{2}} \cdot \frac{\sqrt{M}}{\sqrt{2 \pi}} \cdot e^{-M / 2[\alpha-(N / M)]^{2}}
\end{aligned}
$$

A estimativa de Bayes para os parâmetros serâ, como foi adotado em 3.2.2, a média da distribuição "a posteriori".

Por (3.29) podemos observar que:

$$
\pi(\alpha \mid y)=\frac{\sqrt{M}}{\sqrt{2 \pi}} \cdot e^{-M / 2[\alpha-(N / M)]^{2}} \quad \because \alpha \cap N\left(\frac{N}{M} ; \frac{1}{M}\right) \quad(3.30 a)
$$

e

$$
\pi(\beta \mid y)=\frac{\sqrt{\mathrm{P}}}{\sqrt{2 \pi}} \cdot \mathrm{e}^{-\mathrm{P} / 2[\beta-(Q / P)]^{2}} \cdot \because \beta \cap N\left(\frac{\mathrm{Q}}{\mathrm{P}} ; \frac{1}{\mathrm{P}}\right)(3.30 \mathrm{~b})
$$

Assim as estimativas de Bayes $\left(\alpha *\right.$ e $\left.\beta^{*}\right)$, para os parâmetros $\alpha$ e $\beta$, e suas respectivas variâncias, serão:

$$
\begin{aligned}
& \alpha *=\frac{N}{M}=\frac{\frac{n}{\sigma_{0}^{2}} a+\frac{\alpha_{0}}{\sigma_{a}^{2}}}{\frac{\mathrm{n}}{\sigma_{0}^{2}}+\frac{1}{\sigma_{\mathrm{a}}^{2}}} \quad \text { ou } \quad \alpha *=\frac{a\left(\frac{\sigma_{0}^{2}}{\mathrm{n}}\right)^{-1}+\alpha_{0}\left(\sigma_{\mathrm{a}}^{2}\right)^{-1}}{\left(\frac{\sigma_{0}^{2}}{\mathrm{n}}\right)^{-1}+\left(\sigma_{\mathrm{a}}^{2}\right)^{-1}} \\
& V(\alpha)=\frac{1}{M}=\frac{1}{\frac{\mathrm{n}}{\sigma_{0}^{2}}+\frac{1}{\sigma_{\mathrm{a}}^{2}}} \quad \text { ou } \quad V(\alpha)=\frac{1}{\left(\frac{\sigma_{0}^{2}}{\mathrm{n}}\right)^{-1}+\left(\sigma_{\mathrm{a}}^{2}\right)^{-1}}
\end{aligned}
$$


De modo semelhante, teremos para $\beta$; a partir de $(3.30 b)$

$$
\begin{gathered}
\beta^{*}=\frac{Q}{P}=\frac{\frac{b \cdot S_{x x}}{\sigma_{0}^{2}}+\frac{\beta_{0}}{\sigma_{b}^{2}}}{\frac{S_{x x}}{\sigma_{0}^{2}}+\frac{1}{\sigma_{b}^{2}}} \text { ou } \quad \beta^{*}=\frac{b\left(\frac{\sigma_{0}^{2}}{s_{x x}^{-1}}+\beta_{0}\left(\sigma_{b}^{2}\right)^{-1}\right.}{\left(\frac{\sigma_{0}^{2}}{s_{x x}^{-1}}+\left(\sigma_{b}^{2}\right)^{-1}\right.} \\
V(\beta)=\frac{1}{P}=\frac{1}{\frac{S_{x x}}{\sigma_{0}^{2}}+\frac{1}{\sigma_{b}^{2}}} \text { ou } \quad v(\beta)=\frac{1}{\left(\frac{\sigma_{0}^{2}}{S_{x x}}\right)^{-1}+\left(\sigma_{b}^{2}\right)^{-1}}
\end{gathered}
$$

Podemos definir Precisão como sendo o recíproco da

variância:

$$
\begin{aligned}
& h_{a}=\frac{1}{\sigma_{a}^{2}}=\left(\sigma_{a}^{2}\right)^{-1} \text { precisão "a priori" do parâmetro } \alpha \\
& h_{b}=\frac{1}{\sigma_{b}^{2}}=\left(\sigma_{b}^{2}\right)^{-1} \text { precisão "a priori" do parânetro } \beta \\
& h_{a}^{\prime}=\frac{n}{\sigma_{o}^{2}}=\left(\frac{\sigma_{0}^{2}}{n}\right)^{-1} \text { precisão amostral sobre } \alpha \\
& h_{b}^{\prime}=\frac{s_{x x}}{\sigma_{0}^{2}}=\left(\frac{\sigma_{0}^{2}}{S_{x x}}\right) \text { precisão amostral sobre } \beta \\
& \text { Desta forma, podemos escrever (3.31a) e (3.31b) das }
\end{aligned}
$$

seguintes maneiras:

$$
\alpha *=\frac{a h_{a}^{\prime}+\alpha_{0} h_{a}}{h_{a}^{\prime}+h_{a}} \quad \text { e } \quad v(\alpha)=\frac{1}{h_{a}^{\prime}+h_{a}}
$$


83.

De maneira semelhante $(3.32 a)$ e $(3.32 b)$ ficarão:

$$
\beta *=\frac{b \cdot h_{b}^{\prime}+\beta_{0} \cdot h_{b}}{h_{b}^{\prime}+h_{a}} \quad \text { e } \quad v(\beta)=\frac{1}{h_{b}^{\prime}+h_{b}}
$$

Estas duas $\bar{u} 1 t$ imas expressões nos mostram fatos inte ressantes sobre as estimativas de Bayes. Embora a situação seja hipotética, porquanto estamos considerando a variância conhecida, os resultados expressam analiticamente resultados esperados pelo método e pelo bom senso:

a) Os estimadores de Bayes (média da distribuição "a posteriori") para os parâmetros são médias aritméticas ponderadas das médias "a priori" e amostral, cujos pesos são as respectivas precisões. Assim, quanto maior a precisão da informação provinda dos dados, maior serā o peso a eles associado; quanto maior a precisão da informação "a priori", maior o peso a ela associado.

b) Quando $\underline{\mathrm{n}}$ aumenta, a precisão amostral sobre $\alpha$ também aumenta, o que faz diminuir a variância "a posteriori", $v(\alpha)$.

c) Quanto maior o número de elementos amostrados e quanto menos concentrados em torno de $\bar{x}$ estiverem os $x_{i}$ amostrados (maior $\mathrm{S}_{\mathrm{xx}}$ ), tanto maior será a precisão amostral sobre $\beta$. Assim, a variância "a posteriori" decrescerá. 


\subsubsection{2 - Uso da Distribuição Conjugada}

Em nosso exemplo de aplicação faremos uso das distr $\underline{i}$ buições conjugadas citadas em 3.2.4 e que passamos a desenvolver pa ra o caso do modelo de regressão linear simples em estudo.

Seja conhecida uma amostra $\left\{\mathrm{y}_{1}\right\}$ de tamanho $\mathrm{n}_{1} \circ$ Partin do de "ignorância" sobre os valores dos parâmetros, obtemos a f.d.p. da distribuição "a posteriori", como mostra a expressão (3.11):

$$
\pi_{1}\left(\alpha, \beta, \sigma^{2} \mid y_{1}\right) \propto\left(\sigma^{2}\right)^{-1 / 2\left(n_{1}+2\right)} \cdot \exp \left[-\frac{1}{2 \sigma^{2}}\left[\begin{array}{r}
v_{1} s_{1}^{2}+n_{1}\left(\alpha-a_{1}\right)^{2}+ \\
+s_{x x}^{\beta}\left(\beta-b_{1}\right)^{2}
\end{array}\right]\right\}
$$

Como vimos, as informações que podemos obter sobre os parâmetros, a partir desta amostra são que:

$$
\begin{gathered}
\alpha \cap N\left(a_{1} ; \frac{\sigma^{2}}{n_{1}}\right) \\
\beta \cap N\left(b_{1} ; \frac{\sigma^{2}}{s_{x x i}^{\prime}}\right) \\
\frac{v_{1} s_{1}^{2}}{\sigma^{2}} \cap x_{\left(v_{1}\right)}^{2}
\end{gathered}
$$

Estas informações podem ser usadas como conhecimento prévio para os novos dados que podemos obter, fazendo uma amostragem de tamanho $\underline{n}$, admitindo que esta seja oriunda da mesma população e cuja verossimilhança è dada por: 
85.

$p\left(y \mid \alpha ; \beta ; \sigma^{2}\right)=\left(2 \pi \sigma^{2}\right)^{-n / 2} \cdot \exp \left\{-\frac{1}{2 \sigma^{2}}\left[v s^{2}+n(\alpha-a)^{2}+s_{x x}(\beta-b)^{2}\right]\right\}$

Combinando $(3.35)$ e (3.36) pelo teorema de Bayes, te remos a f.d.p. da distribuição "a posteriori":

$\pi_{2}\left(\alpha ; \beta ; \sigma^{2} \mid y_{1} ; y\right) \propto \pi_{1}\left(\alpha, \beta, \sigma^{2} \mid y_{1}\right) \cdot p\left(y \mid \alpha, \beta, \sigma^{2}\right)$
$\pi_{2}\left(\alpha ; \beta ; \sigma^{2} \mid y_{1} ; y\right) \propto\left(\sigma^{2}\right)^{-1 / 2\left(n_{1}+n+2\right)} \cdot \exp \left\{\begin{array}{c}{\left[\begin{array}{l}v_{1} s_{1}^{2}+v s^{2}+ \\ +n_{1}\left(\alpha-a_{1}\right)^{2}+n(\alpha-a)^{2}+1 \\ 2 \sigma^{2} \\ +s_{x x}^{1}\left(\beta-b_{1}\right)^{2}+ \\ +s_{x x}(\beta-b)^{2}\end{array}\right]}\end{array}\right] ;(3$,

Pelo que foi visto em 3.2.4, o uso de distribuição "a priori" conjugada com a verossimilhança da amostra, conduz à distri buição "a posteriori" com f.d.p. da mesma forma.

Assin:

$\pi_{2}\left(\alpha ; \beta ; \sigma^{2} \mid y_{1} ; y\right) \propto\left(\sigma^{2}\right)^{-1 / 2\left(n_{2}+2\right)} \cdot \exp \left\{-\frac{1}{2 \sigma^{2}}\left[\begin{array}{c}v_{2} s_{2}^{2}+n_{2}\left(\alpha-a_{2}\right)^{2}+ \\ +s_{x x}^{\prime \prime}\left(\beta-b_{2}\right)^{2}\end{array}\right]\right\}$

Para podermos comparar (3.38) com (3.37), façamos al gumas passagens algébricas dentro dos colchetes do expoente desta ư $\underline{1}$ tima expressão (3.37):

$$
\begin{aligned}
E= & v_{1} s_{1}^{2}+v s^{2}+n_{1}\left(\alpha-a_{1}\right)^{2}+n(\alpha-a)^{2}+s_{x x}^{\prime}\left(\beta-b_{1}\right)^{2}+s_{x x}(\beta-b)^{2} \\
= & v_{1} s_{1}^{2}+v s^{2}+n_{1} \alpha^{2}-2 n_{1} a_{1} \alpha+n_{1} a_{1}^{2}+n \alpha^{2}-2 n a \alpha+n a^{2}+s_{x x}^{\prime} \beta^{2}- \\
& -2 s_{x x_{1}}^{\prime} b_{1} \beta+s_{x x_{1}^{\prime}}^{\prime} b_{1}^{2}+s_{x x} \beta^{2}-2 s_{x x} b \beta+s_{x x} b^{2}
\end{aligned}
$$


86.

$$
\begin{aligned}
& E=v_{1} s_{1}^{2}+v s^{2}+\left(n_{1}+n\right) \alpha^{2}-2\left(n_{1} a_{1}+n a\right) \alpha+n_{1} a_{1}^{2}+n a^{2}+\left(s_{x x}^{1}+s_{x x}\right) \beta^{2}- \\
& -2\left(S_{x x_{1}}^{\prime} b_{1}+S_{x x} b\right) B+S_{x x_{1}}^{\prime} b_{1}^{2}+S_{x x^{2}} b^{2},
\end{aligned}
$$

fazendo: $\quad \mathrm{n}_{2}=\mathrm{n}_{1}+\mathrm{n}$

$$
\begin{aligned}
n_{2} a_{2} & =n_{1} a_{1}+n a \\
s_{x x}^{\prime \prime} & =s_{x x}^{\prime}+s_{x x} \\
s_{x x}^{\prime \prime} \cdot b_{2} & =s_{x x}^{\prime} b_{1}+s_{x x}^{b}
\end{aligned}
$$

e completando os quadrados, teremos:

$$
\begin{aligned}
E=v_{1} s_{1}^{2} & +v s^{2}+n_{1} a_{1}^{2}+n a^{2}+s_{x x_{1}^{\prime}}^{\prime} b_{1}^{2}+s_{x x} b^{2}+n_{2}\left(\alpha-a_{2}\right)^{2}-n_{2} a_{2}^{2}+ \\
& +s_{x x}^{\prime \prime}\left(\beta-b_{2}\right)^{2}-s_{x x_{2}^{\prime \prime}}^{\prime \prime} b_{2}^{2} .
\end{aligned}
$$

Logo:

$$
\begin{array}{r}
\mathrm{v}_{2} \mathrm{~s}_{2}^{2}=\mathrm{v}_{1} \mathrm{~s}_{1}^{2}+v \mathrm{~s}^{2}+\mathrm{n}_{1} \mathrm{a}_{1}^{2}+n \mathrm{a}^{2}+\mathrm{s}_{\mathrm{xx}}^{1} \mathrm{~b}_{1}^{2}+\mathrm{s}_{\mathrm{xx}} \mathrm{b}^{2}-\mathrm{n}_{2} \mathrm{a}_{2}^{2}-\mathrm{s}_{\mathrm{xx}}^{\prime \prime} \mathrm{b}_{2}^{2} \\
\text { Desta forma, temos os elementos para tirar conclusões }
\end{array}
$$
de como nosso conhecimento "a priori", formalizado por (3.35) foi mo dificado pela evidência da amostra atual (3.36) e evidenciado pela f.d.p. "a posteriori" em (3.38).

A expressão (3.38) è da mesma forma que (3.11) e,por tanto, todas as conclusões desenvolvidas de 3.3 .2 são válidas para este caso e resumidas assim: 
87.

a) F.D.P. marginais "a posteriori" para os parâmetros:

$$
\begin{aligned}
& \pi_{2}\left(\alpha \mid y_{1} ; y\right)=\frac{\sqrt{n_{2} \cdot s_{2} n_{2}^{-2}}}{B\left(\frac{n_{2}}{2}-1 ; \frac{1}{2}\right)}\left[S_{2}^{2}+n_{2}\left(\alpha-a_{2}\right)^{2}\right]^{-1 / 2\left(n_{2}-1\right)} \quad \text { (3.40a) } \\
& \pi_{2}\left(\beta \mid y_{1} ; y\right)=\frac{\sqrt{S_{x x}^{\prime \prime}} \cdot S_{2}^{n_{2}-2}}{B\left(\frac{n_{2}}{2}-1 ; \frac{1}{2}\right)}\left[S_{2}^{2}+S_{x x}^{\prime \prime}\left(\beta-b_{2}\right)^{2}\right]^{-1 / 2\left(n_{2}-1\right)} \quad(3.40 b) \\
& \pi_{2}\left(\sigma^{2} \mid y_{1} ; y\right)=\frac{s_{2}^{n_{2}-2}}{2^{\left(n_{2} / 2\right)-1} \cdot \Gamma\left(\frac{n_{2}}{2}-1\right)}\left(\sigma^{2}\right)^{-n_{2} / 2} \cdot e^{-S_{2}^{2} / 2 \sigma^{2}}(3.40 c) \\
& \pi_{2}\left(\alpha ; \beta \mid y_{1} ; y\right) \propto\left[s_{2}^{2}+n_{2}\left(\alpha-a_{2}\right)^{2}+s_{x x}^{\prime \prime}\left(\beta-b_{2}\right)^{2}\right]^{-n_{2} / 2}
\end{aligned}
$$

onde:

$$
\begin{aligned}
& \mathrm{a}_{2}=\frac{1}{\mathrm{n}_{2}}\left(\mathrm{n}_{1} \mathrm{a}_{1}+\mathrm{na}\right) \\
& \mathrm{b}_{2}=\frac{1}{\mathrm{~s}_{\mathrm{xx}}^{\prime \prime}}\left(\mathrm{s}_{\left.\mathrm{xx} \mathrm{x}_{1}^{\prime} \mathrm{b}_{1}+\mathrm{s}_{\mathrm{xx}} \mathrm{b}\right)}\right. \\
& \mathrm{s}_{2}^{2}=\mathrm{v}_{2} \mathrm{~s}_{2}^{2}
\end{aligned}
$$

e, por (3.39): 
$s_{2}^{2}=\frac{1}{v_{2}}\left(v_{1} s_{1}^{2}+v s^{2}+n_{1} a_{1}^{2}+n a^{2}+s_{x x_{1}}^{\prime} b^{2}+s_{x x} b^{2}-n_{2} a_{2}^{2}-s_{x x}^{\prime \prime} b_{2}^{2}\right)$

b) Estimadores de Bayes para os parāmetros e suas variāncias:

$$
\text { As distribuições "a posteriori" para os parâmetros } \alpha
$$

e $\beta$ serão:

$$
\begin{aligned}
& \alpha \cap N\left(a_{2} ; \frac{\sigma^{2}}{n_{2}}\right) \\
& \beta \cap N\left(b_{2} ; \frac{\sigma^{2}}{S_{x x}^{\prime \prime}}\right)
\end{aligned}
$$

Assim:

$$
\alpha^{*}=a_{2}=\frac{n_{1} a_{1}+n a}{n_{1}+n} \quad \text { e } \quad v(\alpha)=\frac{\sigma^{2}}{n_{2}}
$$

e

$$
\beta^{*}=b_{2}=\frac{S_{x x_{1}^{\prime} b_{1}+S_{x x} b}}{S_{x x}^{\prime \prime}} \text { e } V(\beta)=\frac{\sigma^{2}}{S_{x x}^{\prime \prime}}
$$

c) Imparcialidade das estimativas de Bayes:

$\operatorname{De}(3.42 a)$ :

$$
\begin{aligned}
E\left(a_{2}\right) & =\frac{1}{n_{2}} E\left(n_{1} a_{1}+n a\right)=\frac{1}{n_{2}}\left[n_{1} E\left(a_{1}\right)+n E(a)\right] \\
& =\frac{1}{n_{2}}\left[n_{1} \alpha+n \alpha\right]=\alpha
\end{aligned}
$$


89.

De $(3.42 b)$ :

$$
\begin{aligned}
& E\left(b_{2}\right)=\frac{1}{S_{x x}^{\prime \prime}} E\left(S_{x x_{1}^{\prime}}^{\prime} b_{1}+S_{x x} b\right)=\frac{1}{S_{x x}^{\prime \prime}}\left[S_{x x^{\prime}}^{\prime} E\left(b_{1}\right)+S_{x x} E(b)\right] \\
& =\frac{1}{S_{x x}^{\prime \prime}}\left[S_{x x}^{\prime} \beta+S_{x x} \beta\right]=\beta \\
& \text { De (3.39): } \\
& v_{2} E\left(s_{2}^{2}\right)=v_{1} E\left(s_{1}^{2}\right)+v E\left(s^{2}\right)+n_{1} E\left(a_{1}^{2}\right)+n E\left(a^{2}\right)+s_{x x}^{1} E\left(b_{1}^{2}\right)+ \\
& +s_{x x} E\left(b^{2}\right)-n_{2} E\left(a_{2}^{2}\right)-S_{x x}^{\prime \prime} E\left(b_{2}^{2}\right) \\
& \mathrm{v}_{2} E\left(\mathrm{~s}_{2}^{2}\right)=\mathrm{v}_{1} \sigma^{2}+\mathrm{v} \sigma^{2}+\mathrm{n}_{1}\left(\frac{\sigma^{2}}{\mathrm{n}_{1}}+\alpha^{2}\right)+\mathrm{n}\left(\frac{\sigma^{2}}{\mathrm{n}}+\alpha^{2}\right)+ \\
& +S_{x x}^{\prime}\left(\frac{\sigma^{2}}{S_{x x}^{\prime}}+\beta^{2}\right)+S_{x x}\left(\frac{\sigma^{2}}{S_{x x}}+\beta^{2}\right)- \\
& -\mathrm{n}_{2}\left(\frac{\sigma^{2}}{\mathrm{n}_{2}}+\alpha^{2}\right)-S_{\mathrm{xx}}^{\prime \prime}\left(\frac{\sigma^{2}}{S_{\mathrm{xx}}^{\prime \prime}}+\beta^{2}\right) \\
& v_{2} E\left(s_{2}^{2}\right)=\left(v_{1}+v\right) \sigma^{2}+\left(n_{1}+n\right) \alpha^{2}+\left(S_{x x}^{1}+S_{x x}\right) \beta^{2}+4 \sigma^{2}- \\
& -\mathrm{n}_{2} \alpha^{2}-\mathrm{S}_{\mathrm{xx}}^{\prime \prime} \beta^{2}-2 \sigma^{2} \\
& v_{2} E\left(s_{2}^{2}\right)=\left(v_{1}+v+2\right) \sigma^{2} \\
& \mathrm{v}_{2} \mathrm{E}\left(\mathrm{s}_{2}^{2}\right)=\mathrm{v}_{2} \sigma^{2} \quad \Longrightarrow \quad \mathrm{E}\left(\mathrm{s}_{2}^{2}\right)=\sigma^{2}
\end{aligned}
$$


d) Intervalos de confiança e testes de hipōteses:

De maneira anāloga ao que foi desenvolvido em 3.3.2.3, podemos deduzir que:

$$
\frac{\alpha-a_{2}}{s\left(a_{2}\right)} \text { tem distribuição de " } t \text { " com } v_{2}=n_{2}-2 \text { graus de liber- }
$$$$
\frac{\beta-b_{2}}{s\left(b_{2}\right)} \text { tem distribuição de " } t \text { " com } v_{2}=n_{2}-2 \text { graus de 1.iber- }
$$

$$
\begin{aligned}
& \frac{\mathrm{v}_{2} \mathrm{~s}_{2}^{2}}{\sigma^{2}} \quad \text { tem distribuição de "qui-quadrado" com } \mathrm{v}_{2}=\mathrm{n}_{2}-2 \\
& \text { graus de liberdade; }
\end{aligned}
$$

onde: $\quad s\left(a_{2}\right)=\sqrt{\hat{v}\left(a_{2}\right)}=\frac{s_{2}}{\sqrt{n_{2}}} \quad$ e $\quad s\left(b_{2}\right)=\sqrt{\widehat{V}\left(b_{2}\right)}=\frac{s_{2}}{\sqrt{s_{x x}^{\prime \prime}}}$ Desta forma, os testes de hipóteses, Intervalos. de Confiança para os parâmetros e Intervalo de Previsão são elaborados da mesma maneira já vista em $3.3 .2 .3,3.3 .2 .4$ e 3.3.2.5. 
91 .

4. COMPARAÇAOO DOS METODOS ATRAVES DE SIMULAÇATO DE DADOS

\section{1 - Obtenção dos Dados}

Usou-se o Computadór IBM 1130 para a simulação dos dạ dos e para os cálculos necessários à anālise estatística, através do programa constante no Apêndice I.

A anālise de regressão pelo método dos Mỉnimos Quadrados foi aplicada aos dados da chamada "Amostra Atual", de tamanhos $n=7, n=11$ e $n=15$.

0 método de Bayes, para a anālise de regressão, se baseou em dados infórmativos, disponíveis previamente através de es tatísticas suficientes, obtidas da chamada "Amostra Prévia" (também gerada por simulação aleatória), de tamanho $\mathrm{n}_{1}=21$, com valores da variável independente (considerada não aleatōria) iguais a: -30; $-30 ;-30 ;-20 ;-20 ;-20 ;-10 ;-10 ;-10 ; 0 ; 0 ; 0 ; 10 ; 10 ; 10 ; 20 ; 20$; 
92.

20 ; 30; 30; 30. As informações "a priori" foram incorporadas à análise atual pela Distribuição Conjugada.

Foram estabelecidos 11 (onze) casos de "experimentos", obedecendo aos seguintes critêrios:

a) Os dados foram gerados segundo os modelos:

$$
Y_{i}=60+0,8 x_{i}+e_{i}^{(I)} \text {, para a "Amostra Prévia" }
$$

e

$$
Y_{j}=60+0,8 x_{j}+e_{j}^{(I I)} \text {, para a "Amostra Atual" }
$$

onde, $x$ é a variável independente, com valores centrados em $x=0$;

$$
\mathrm{e}_{i}^{(I)} \cap \mathrm{N}\left(0 ; \sigma_{1}^{2}\right) \quad \mathrm{e}^{\cdot} \quad \mathrm{e}_{j}^{(\mathrm{II})} \cap \mathrm{N}\left(0 ; \sigma_{2}^{2}\right)
$$

b) Os 11 casos de simulação foram subdivididos em dois grupos, conforme os dados das amostras "Atual" e "Prévia" fossem gerados com variâncias residuais iguais ou diferentes;

$$
\begin{array}{ll}
\text { Grupo I: } & \sigma_{1}^{2}=\sigma_{2}^{2} \\
\text { Grupo II: } & \sigma_{1}^{2} \neq \sigma_{2}^{2}
\end{array}
$$

c) A simulação dos dados amostrais, para cada um dos 11 casos, foi feita conforme os tamanhos das amostras e valores das variân-. cias residuais, constantes na Tabela 1 . 
93.

Tabela 1 - Especificação dos 11 casos de simulação dos dados amostrais conforme o tamanho da amostra e variância residual.

\begin{tabular}{ccccccc}
\hline & & \multicolumn{2}{r}{ Amostra } & "Prëvia" & & \multicolumn{2}{c}{ Amostra "Atual" } \\
\cline { 3 - 4 } \cline { 5 - 6 } GRUPO & CASO & $\mathrm{n}_{1}$ & $\sigma_{1}^{2}$ & $\mathrm{n}$ & $\sigma_{2}^{2}$ \\
\hline I & 19 & 21 & 36 & 7 & 36 \\
I & 29 & 21 & 36 & 11 & 36 \\
I & 39 & 21 & 36 & 15 & 36 \\
I & 49 & 21 & 144 & 7 & 144 \\
I & 59 & 21 & 144 & 11 & 144 \\
I & 69 & 21 & 144 & 15 & 144 \\
& & & & & \\
II & 79 & 21 & 36 & 7 & 144 \\
II & 89 & 21 & 36 & 11 & 144 \\
II & 99 & 21 & 36 & 15 & 144 \\
II & 109 & 21 & 144 & 7 & 36 \\
II & 119 & 21 & 144 & 11 & 36 \\
\hline
\end{tabular}

d) Os valores da variāvel $x_{j}$ utilizados, de acordo com o tamanho da Amostra "Atual", foram:

$$
\begin{aligned}
& \mathrm{n}=7:-30 ;-20 ;-10 ; 0 ; 10 ; 20 ; 30 \\
& \mathrm{n}=11:-30 ;-20 ;-20 ;-10 ;-10 ; 0 ; 10 ; 10 ; 20 ; 20 ; 30 \\
& \mathrm{n}=15:-30 ;-30 ;-20 ;-20 ;-10 ;-10 ; 0 ; 0 ; 0 ; 10 ; 10 ; 20 ; 20 ; \\
& \quad 30 ; 30
\end{aligned}
$$

e) A simulação dos dados amostrais e a anālise de regressão pe los dois métodos foram processadas, repetindo-se duzentas vezes este procedimento para cada um dos 11 casos. 
94.

4.2 - Resultados

Com os dados gerados foram obtidos:

a) A anālise de regressão, segundo o modelo $Y_{i}=\alpha+\beta x_{i}+u_{i}$, para cada uma das duzentas repetições, em cada caso, determinando-se as estimativas dos parâmetros, as estimativas das variâncias des tas estimativas, os intervalos de confiança para os parâmetros ao ni vel de confiança de $95 \%$.

Nas amostras "Prévia" e "Atual" foram feitas as anālises pelo método dos Mínimos Quadrados. Para a análise pelo método de Bayes utilizaram-se as expressões obtidas em 3.3.3.2, de Distribuições Conjugadas.

b) Os valores médios (das dúzentas repetições) das estimativas dos parâmetros, das estimativas das variâncias das estimativas dos parâmetros e dos limites dos intervalos de confiança dos parâmetros, para cada caso considerado.

c) 0 erro quadrático médio das estimativas dos parâmetros para cada um dos 11 casos considerados e para os dois métodos.

0 erro quadrätico médio é aqui definido por:

$$
\begin{aligned}
& \mathrm{EQM}_{\alpha}=\frac{1}{200} \cdot \sum_{i=1}^{200}\left(\widehat{\alpha}_{i}-60\right)^{2}, \text { para as estimativas de } \alpha ; \\
& \mathrm{EQM}_{\beta}=\frac{1}{200} \sum_{i=1}^{200}\left(\widehat{\beta}_{i}-0,8\right)^{2}, \text { para as estimativas de } \beta .
\end{aligned}
$$


d) A frequência das estimativas dos parâmetros em 30 pequenos intervalos de amplitude 1,00 e entre os valores 40,50 e 70,50 para $\alpha$; de amplitude 0,40 e entre os valores 0,22 e 1,42 para $\beta$, em cada um dos casos.

De posse dos dados gerados pela simulação, e feitas as anālises de regressão pelos dois métodos considerados, os resultados obtidos podem ser observados nas Tabelas de 2 a 5 .

A Tabela 2 mostra os valores médios obtidos em 200 repetições, para cada caso do Grupo I. Os dados para a Amostra "Pré via" e Amostra "Atual" foram gerados com erros residuais de mesma variância .

A Tabela 3 mostra os valores mëdios obtidos em 200 repetições, para cada caso do Grupo II. Os dados para as Amostras "Prévia" e "Atual" foram gerados com erros residuais de variâncias diferentes 。

Nas Tabelas 4 e 5 são apresentadas as distribuições de frequências das estimativas de Mínimos Quadrados nas Amostras "Atuais" e estimativas de Bayes para os parâmetros $\alpha$ e $\beta$, nos casos do Grupo II.

E valioso ressaltar que as estimativas de Bayes para os casos do Grupo II foram obtidas sob a hipótese de que os dados das amostras "Prévia" e "Atual" tivessem a mesma variância residual, embora, para este grupo, tenham sido consideradas diferentes. Tal procedimento visa testar a robustez do método quando é violada a hi pótese. 


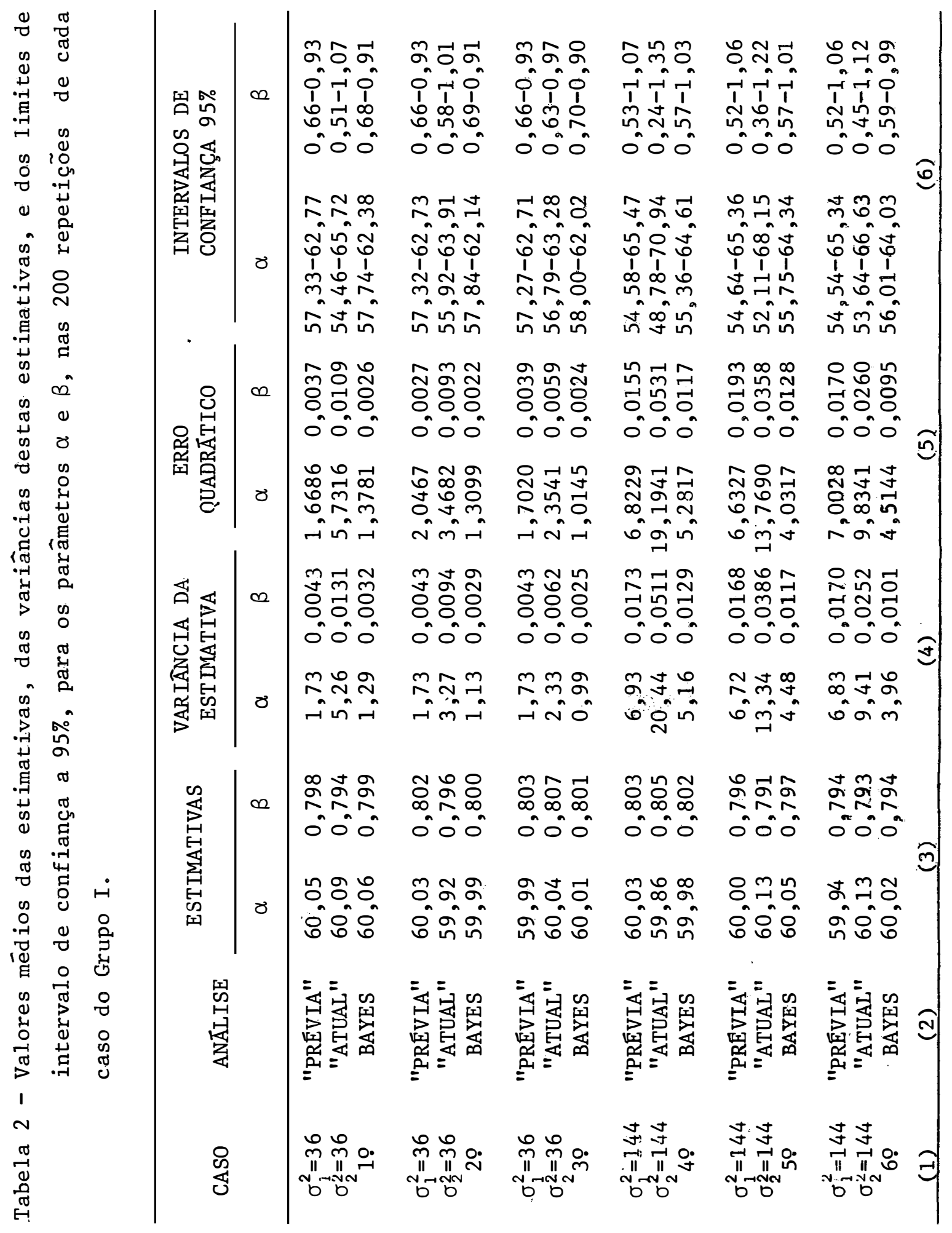


97.

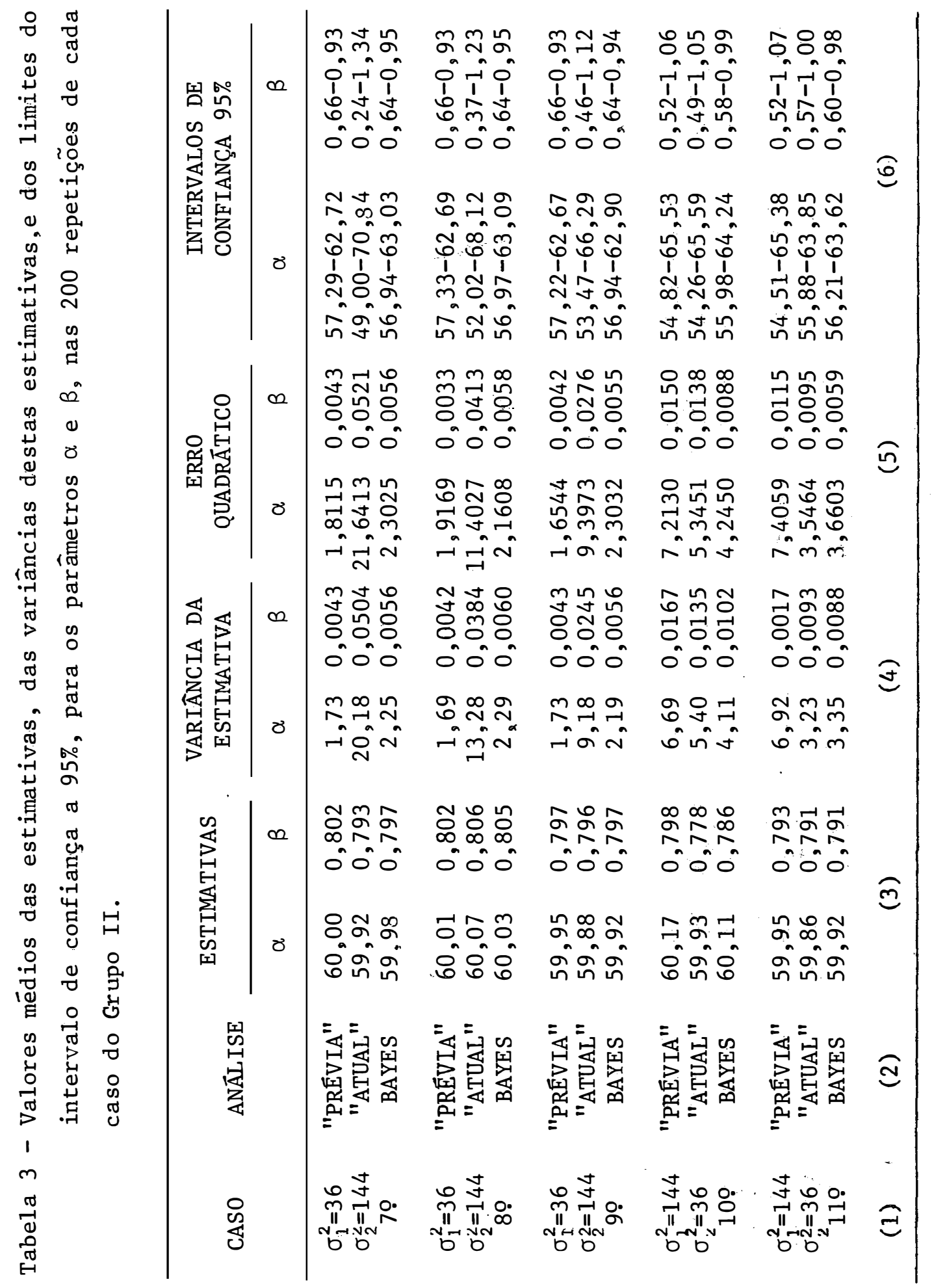


98.

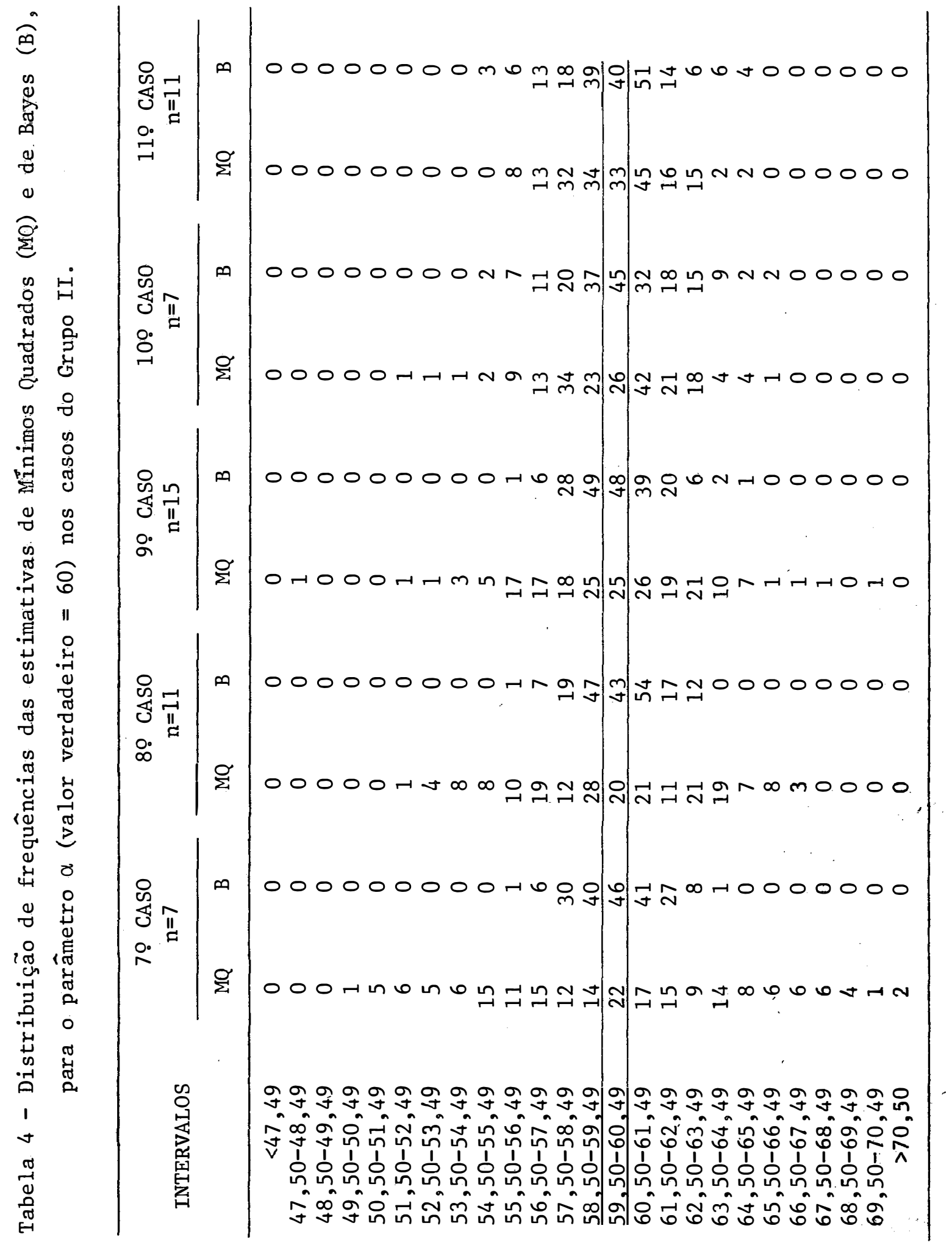




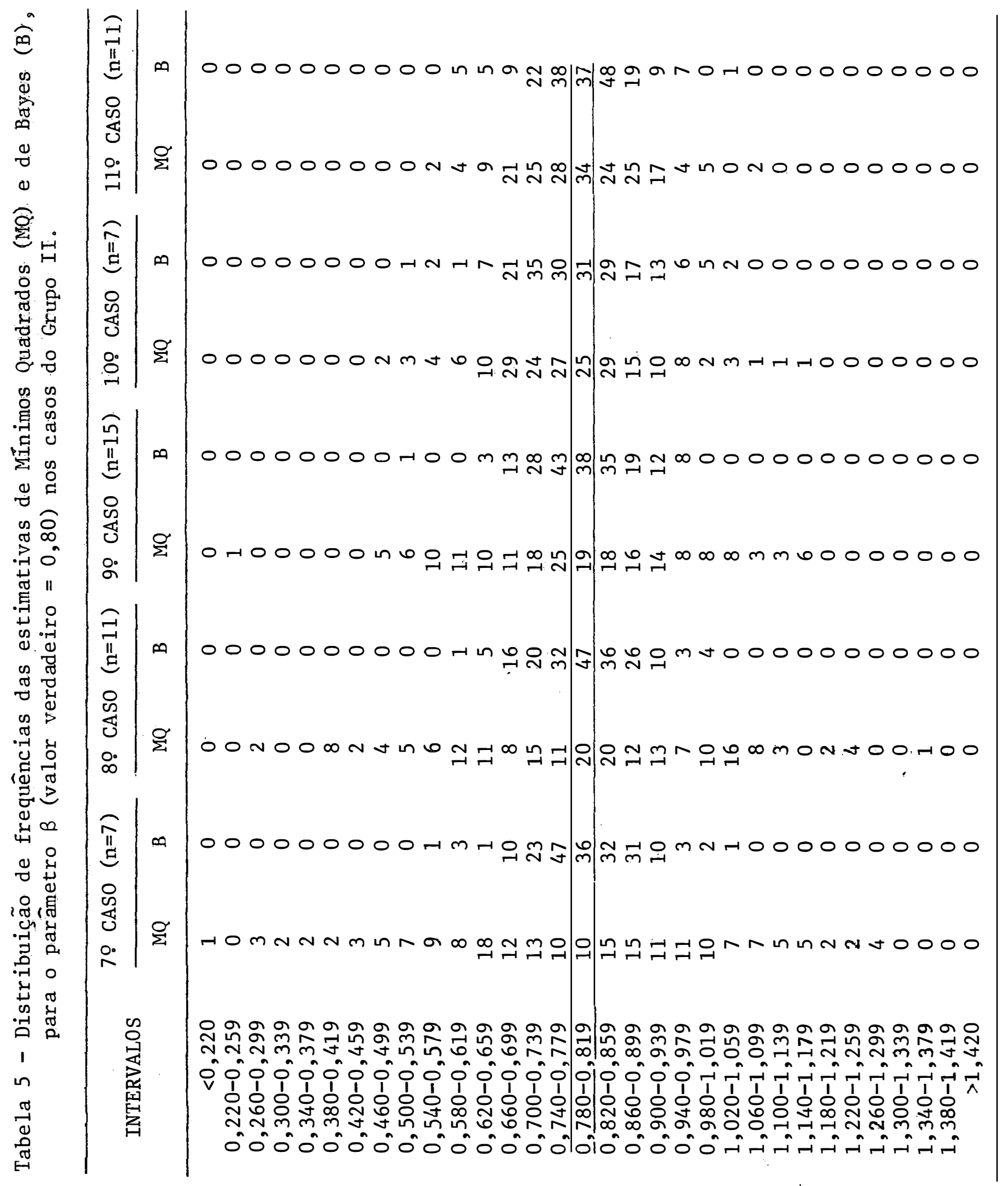


100 。

\section{3 - Exemplo Ilustrativo}

Sorteada uma das duzentas repetições do 19 caso, os dados obtidos por simulações e os resultados correspondentes da aná lise de regressão são apresentados a seguir.

\subsection{1 - Amostra "Prēvia"}

Os dados gerados pelo modelo $\mathrm{Y}_{\mathrm{i}}=60+0,8 \mathrm{x}_{\mathrm{i}}+\mathrm{e}_{\mathrm{i}}^{(\mathrm{I})}$, $\operatorname{com} e_{i}^{(I)}$ distribuỉdo normalmente com média zero e variância $\sigma_{1}^{2}=36$, encontram-se na Tabela 6 .

Tabela 6 - Dados do 10 caso, simulados para a "Amostra Prévia", de tamanho $\mathrm{n}_{1}=21$.

\begin{tabular}{rcrccc}
\hline$x_{i}$ & $Y_{i}$ & $x_{i}$ & $y_{i}$ & $x_{i}$ & $Y_{i}$ \\
\hline-30 & 42,6033 & -10 & 56,3636 & 10 & 67,1884 \\
-30 & 31,3419 & -10 & 50,2652 & 20 & 82,0902 \\
-30 & 41,3210 & 0 & 62,8533 & 20 & $70,327.9$ \\
-20 & 46,5628 & 0 & 63,7680 & 20 & 77,8651 \\
-20 & 45,1694 & 0 & 65,2851 & 30 & 86,7205 \\
-20 & 39,3568 & 10 & 78,9015 & 30 & 81,8474 \\
-10 & 42,2986 & 10 & 73,3064 & 30 & 88,0109 \\
\hline
\end{tabular}

O método dos Mínimos Quadrados fornece o seguinte con junto de estatisticas suficientes $\left(n_{1} ; a_{1} ; b_{1} ; \dot{s}_{1}^{2} ; S_{X X}^{\prime}\right)$ :

$$
\begin{array}{lll}
\mathrm{n}_{1}=21 & \mathrm{~b}_{1}=0,8247 & \mathrm{~s}_{\mathrm{xx}}^{1}=8400 \\
a_{1}=61,59 & \mathrm{~s}_{1}^{2}=25,20 &
\end{array}
$$




\subsection{2 - "Amostra Atual" e Estimativas de Bayes}

Os dados gerados pelo modelo $\mathrm{Y}_{\mathrm{j}}=60+0,8 \mathrm{x}_{\mathrm{i}}+\mathrm{e}_{\mathrm{j}}^{(\text {II })}$, $\operatorname{com} e_{j}^{(I I)}$ distribuído normalmente com média zero e variância $\sigma_{2}^{2}=36$, encontram-se na Tabela 7 .

Tabela 7 - Dados do 19 caso, simulados para a "Amostra Atual"。

\begin{tabular}{rccc}
\hline$x_{j}$ & $Y_{j}$ & $x_{j}$ & $Y_{j}$ \\
\hline-30 & 36,1354 & 10 & 69,4674 \\
-20 & 40,6495 & 20 & 81,8488 \\
-10 & 47,6850 & 30 & 90,3001 \\
0 & 74,0876 & & \\
\hline
\end{tabular}

0 método dos Mínimos Quadrados para a "Amostra Atual" e o método de Bayes forneceram os seguintes resultados:

a) Estimativas dos parâmetros e as estimativas de suas variạncias :

"Amostra Atual"

$$
\begin{array}{ll}
\mathrm{a}=62,88 & \hat{\mathrm{V}}(\mathrm{a})=5,1771 \\
\mathrm{~b}=0,9524 & \nabla(\mathrm{b})=0,0129
\end{array}
$$

Estimativas de Bayes

$$
\begin{aligned}
& a_{2}=61,91 \quad \hat{V}\left(a_{2}\right)=0,96 \\
& \mathrm{~b}_{2}=0,856.7 \quad \hat{\mathrm{v}}\left(\mathrm{b}_{2}\right)=0,0024
\end{aligned}
$$


As equações das retas estimadas por Mínimos Quadrados e pelo método de Bayes acham-se representadas na Figura 5, comparadas com a verdadeira reta de regressão.

As distribuições "a posteriori" para os parâmetros $\alpha$ e $\beta$, baseadas no método de Bayes, estão representadas na Figura 6 , pelas funções de densidade de probabilidade - $\pi(\alpha \mid y)$ e $\pi(\beta \mid y)$ - e comparadas com aquelas obtidas por Mínimos Quadrados aplicado à amostra "Atual" - $\mathrm{p}(\mathrm{a})$ e $\mathrm{p}(\mathrm{b})$ - e obtidas a partir das expressões $(3.13) \mathrm{e}(3.15)$.

b) Intervalos de confiança para os parâmetros:

Os intervalos de confiança para $\alpha$ e $\beta$ ao nỉvel de.95\% foram determinados. Utilizando apenas a amostra "Atual", encontramos:

$$
\begin{aligned}
57,03<\alpha<68,73 \\
0,66<\beta<1,24
\end{aligned}
$$

O método bayesiano fornece:

$$
\begin{gathered}
58,89<\alpha<63,93 \\
0,75<\beta<0,96
\end{gathered}
$$

c) Intervalos de confiança para $E\left(Y_{h}\right)$ e intervalo de previsão para $\mathrm{Y}_{\mathrm{h}}$ :

Para estimar o valor de $\mathrm{Y}_{\mathrm{h}}$ que corresponde a um : valor de $x_{h}$ que não aparece na amostra, fazemos

$$
\hat{\mathrm{Y}}_{\mathrm{h}}=\hat{\alpha}+\widehat{\beta} \mathrm{x}_{\mathrm{h}}
$$


e cuja precisão é avaliada pelo intervalo de previsão, cujos limites, ao nîvel de confiança de $95 \%$, são dados por:

$$
\widehat{\mathrm{Y}}_{\mathrm{h}} \pm 15,477 \sqrt{\frac{8}{7}+\frac{\mathrm{x}_{\mathrm{h}}^{2}}{2800}}
$$

ao se aplicar (3.24) à amostra "Atual"。

0 intervalo de Bayes, ao mesmo nỉvel de confiança, tem limites dados por:

$$
\widehat{\mathrm{P}}_{\mathrm{h}} \pm 12,377 \sqrt{\frac{29}{28}+\frac{\mathrm{x}_{\mathrm{h}}^{2}}{11200}}
$$

0 intervalo de confiança para $E\left(Y_{h}\right)=\alpha+\beta x_{h}$, ao nỉvel de confiança de $95 \%$, tem limites dados por:

$$
\widehat{\mathrm{Y}}_{\mathrm{h}} \pm 15,477 \sqrt{\frac{1}{7}+\frac{\mathrm{x}_{\mathrm{h}}^{2}}{2800}}
$$

no caso de se aplicar (3.23) à amostra "Atual", enquanto que o intervalo, segundo Bayes, tem limites dados por:

$$
\widehat{\mathrm{Y}}_{\mathrm{h}} \pm 12,377 \sqrt{\frac{1}{28}+\frac{\mathrm{x}_{\mathrm{h}}^{2}}{11200}} \text {. }
$$

Estes resultados podem ser observados na Figura 7.

d) Distribuição conjunta das estimativas dos parâmetros e da variância:

Por (3.14) e (3.12) encontramos a f.d.p.da distribuição conjunta para a estimativa do parâmetro e da variância,e que foram locadas em gráficos de isodensidade, como mostram as Figuras 8 e 9. 


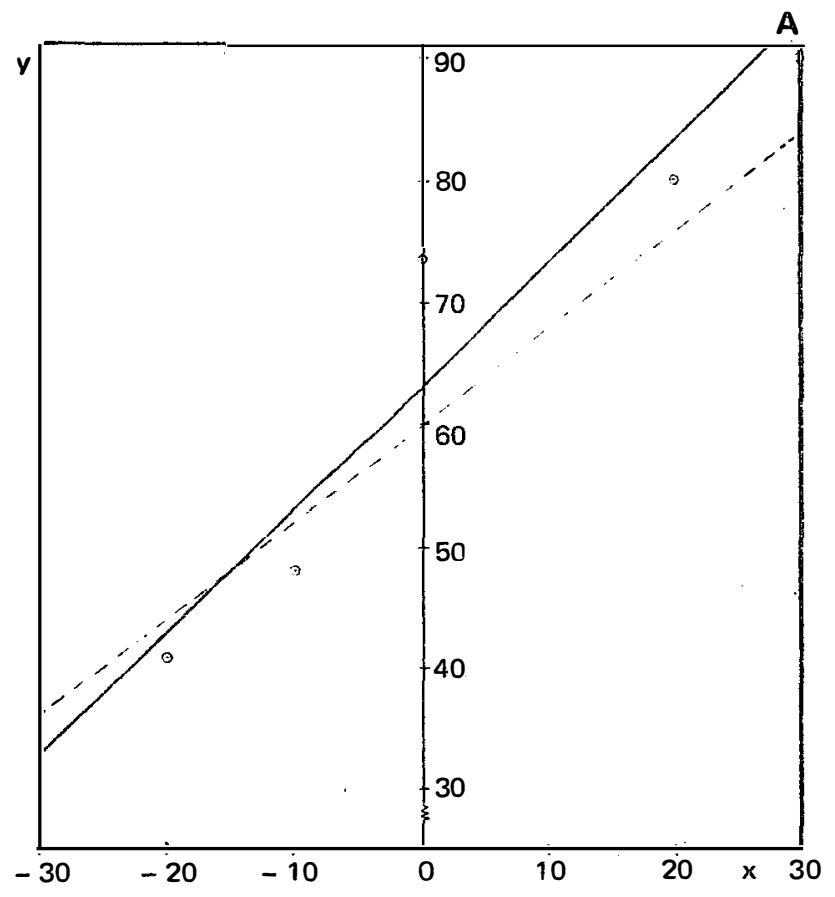

104 .

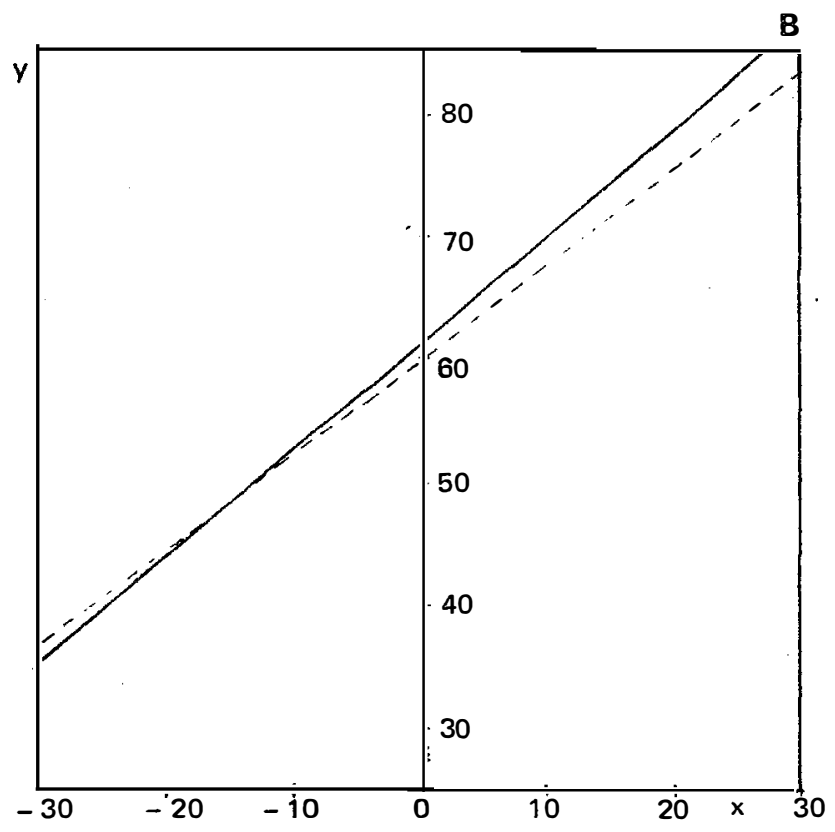

Figura 5 - Representação gräfica da reta ajustada (-) e a verdadeira reta de regressão (--) .

A- Reta ajustada por Mínimos Quadrados: $Y=62,88+0,9524 x$

B- Reta ajustada pelo Método de Bayes: $Y=61,91+0,8567 x$ 
105 .
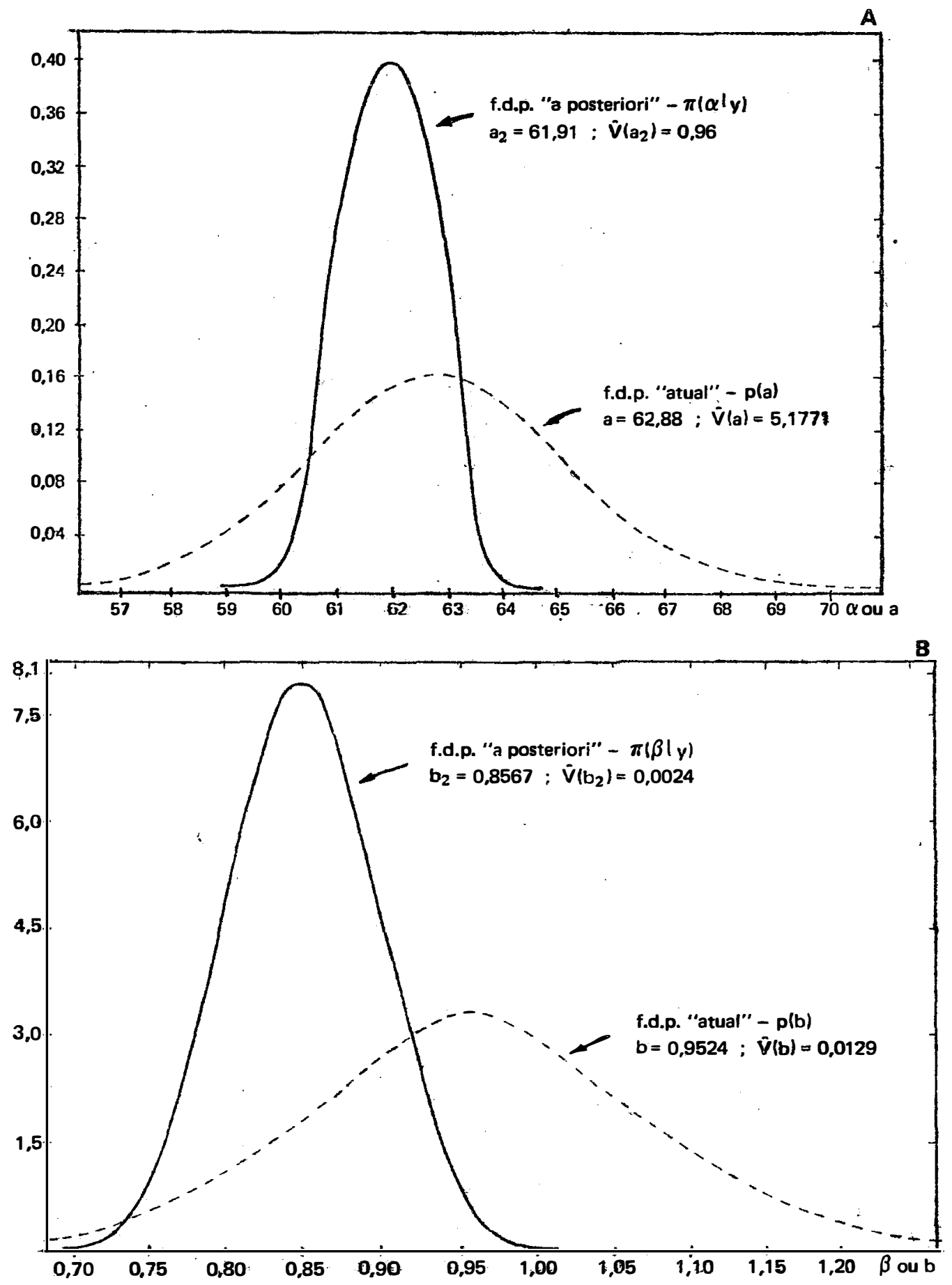

Figura 6 - Distribuição "a posteriori" para os parâmetros $\alpha$ e $B$ da equação de regressão (-) comparada com a estimativa da f.d.p., obtida na amostra "Atual" pelo método de Mínimos Quadrados (---). 


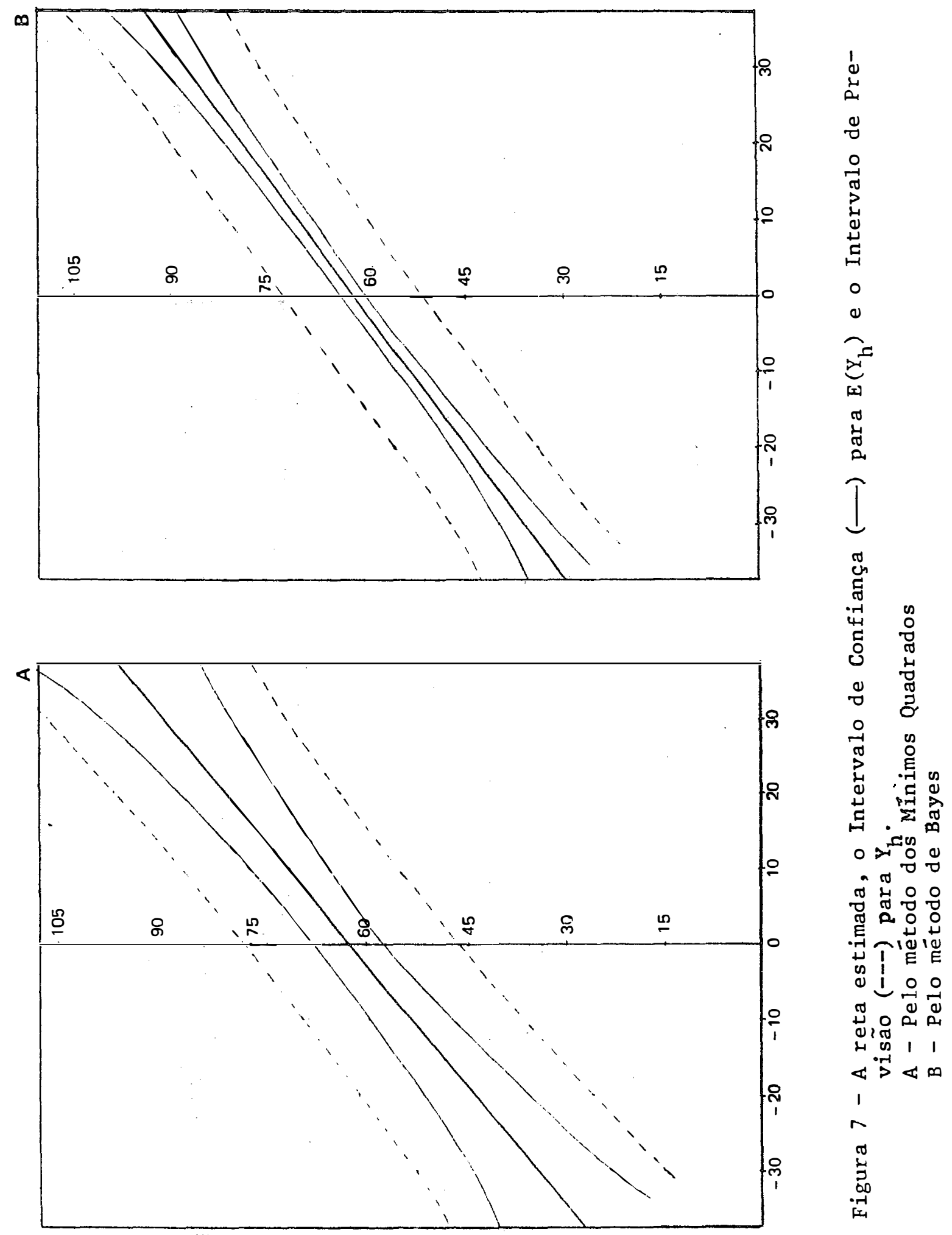


107.
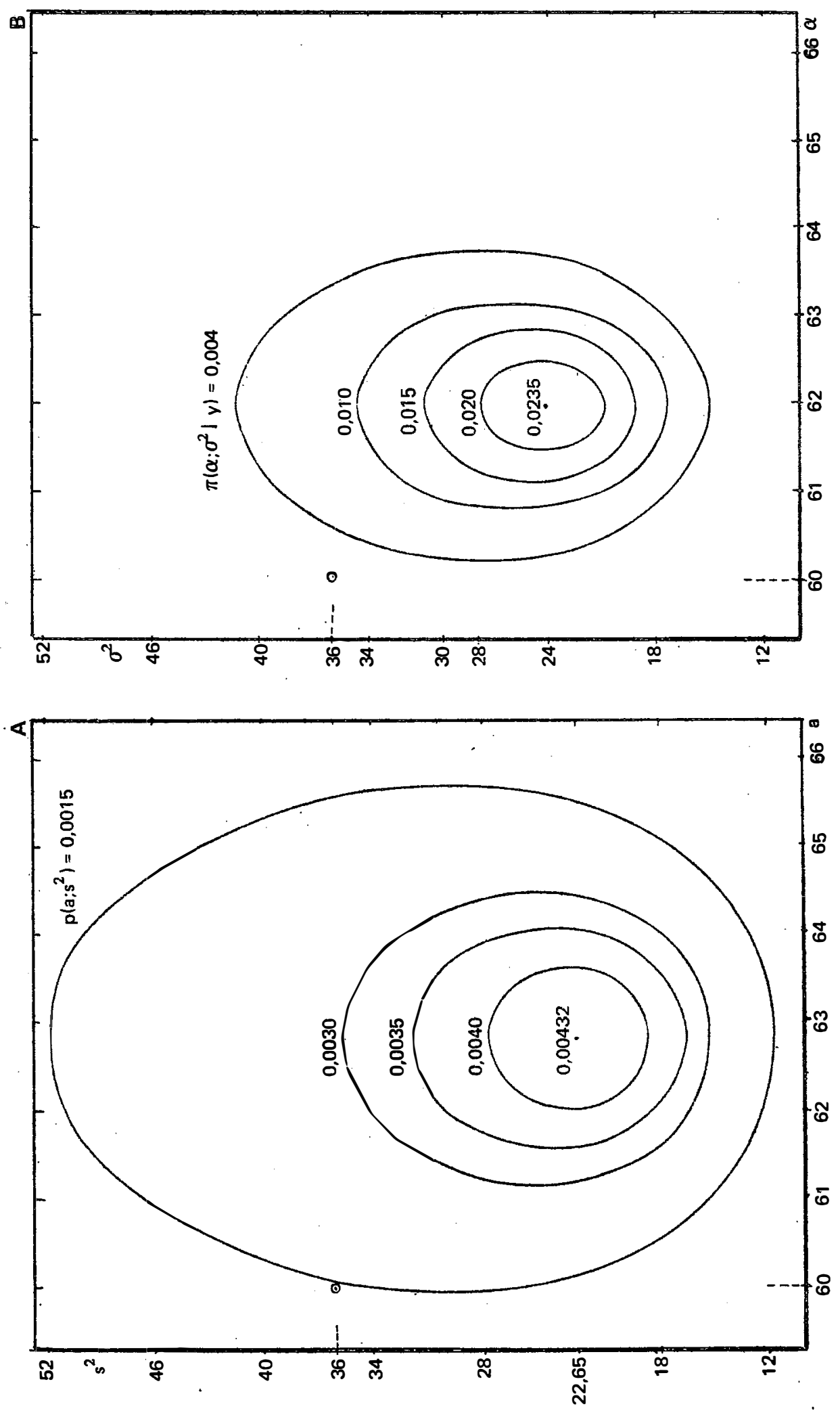

กู

ลั่

III

क क

(1) 0

क

$\infty$ a

ชิ ธิ

11

ब

$\pi$

苂

a 2

๑त

० भे ले

ชठ유

ข 0 0

प्रा०

$\stackrel{\pi}{\pi}$

可院

范是

0 मे

ฉ.

$\stackrel{2}{\rightarrow}$

ग. 0

4

का

ปั

䆑的

-

.

ฮิ ๆ

ㅇ. 용

- ElO

o

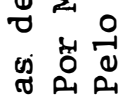

弯 1

ठ઼ 4 
108.
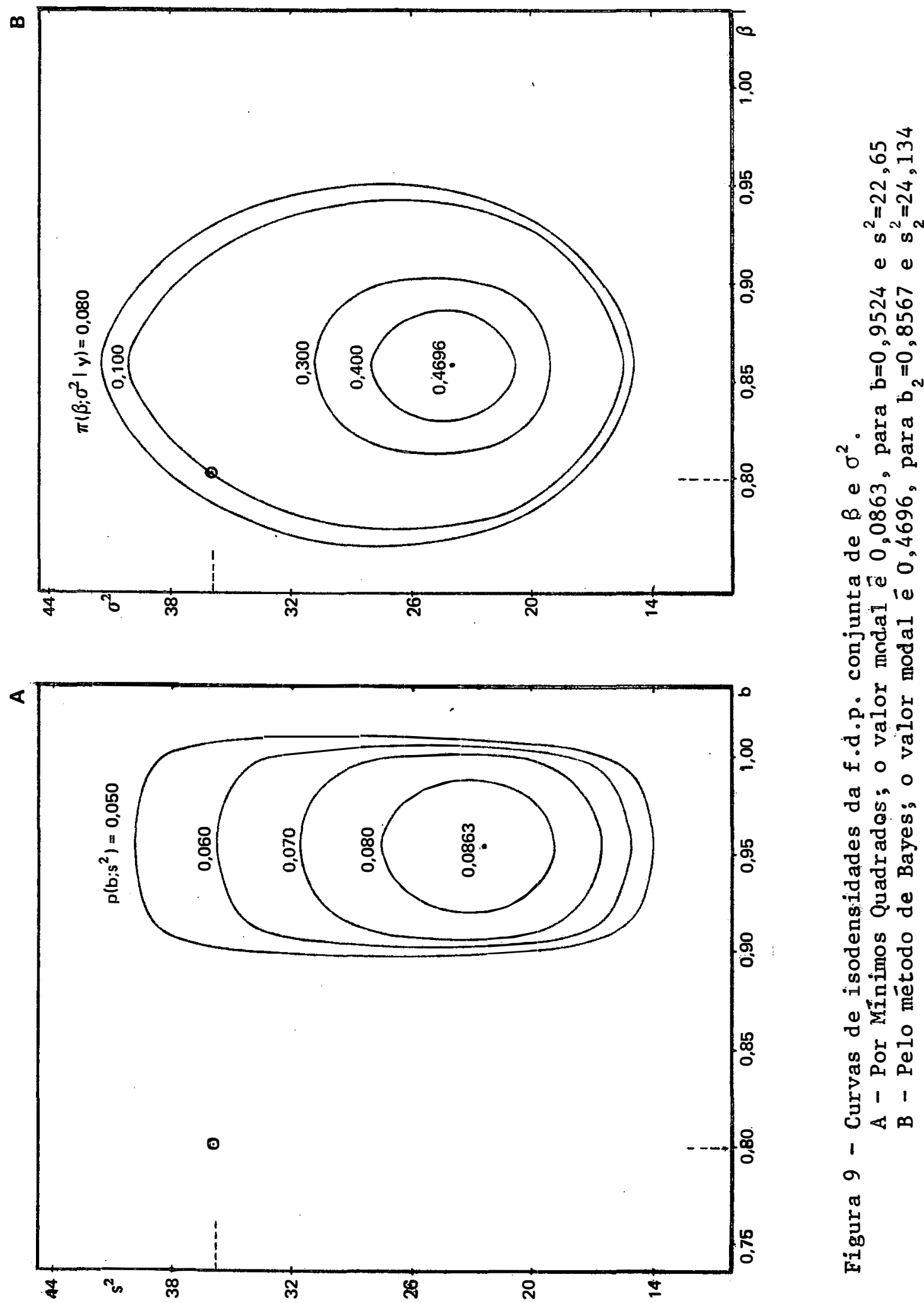


\section{4 - Discussão dos Resultados}

Ao analisar os resultados obtidos, convém salientar que os dados disponíveis para informação "a priori" foram também sí mulados, de modo a considerar todas as situações possíveis quanto à qualidade da informação sobre os verdadeiros valores dos parâmetros. Apenas foi considerado fixo o tamanho da amostra "prévia" $\left(n_{1}=21\right)$, maior que o da amostra "atual" (disponível para análise pelo método dos Mínimos Quadrados).

No presente estudo, a anālise de regressão pelo méto do de Bayes utilizou apenas o caso das Distribuições Conjugadas para incorporar informação "a priori" às informações contidas na amos tra "atua1" e desenvolvido em 3.3.3.2.

\subsection{1 - Estimativas dos parâmetros}

A coluna (3) das Tabelas 2 e 3 mostra que as estimativas de Minimos Quadrados e de Bayes para os parâmetros $\alpha$ e $\beta$ da equação de regressão tendem aos valores verdadeiros $(\alpha=60$ e $\beta=0,80)$, pelos valores médios obtidos nas 200 repetições de cada caso. Isto se explica pela lei dos Grandes Números e pelo fato de os estimadores serem imparciais.

Os estimadores de Bayes para o caso em questão: são dados por (3.42): 


$$
\begin{aligned}
& \alpha *=a_{2}=\frac{n_{1} a_{1}+n a}{n_{1}+n} \\
& \beta *=b_{2}=\frac{S_{x x_{1}^{\prime} b_{1}+S_{x x} b}^{S_{x x}^{\prime}+S_{x x}} .}{} .
\end{aligned}
$$

Estas expressões mostram que o estimador de Bayes ē média ponderada com pesos positivos e, portanto, fornecerá estimatí va com valor entre os valores ponderados e mais próximo daquele de maior peso.

Em 4.3, o exemplo particular do 10 caso estudado mos tra que se pudéssemos contar, no momento, com apenas uma pequena amostra ( $n=7)$ e dispondo de informações prévias mais confiäveis $\left(n_{1}=\right.$ =21), o método de Bayes melhoraria sensivelmente as estimativas, principalmente a do parâmetro $\beta$. Estes resultados podem ser visuali zados na Figura 6, com a distribuição "a posteriori" de Bayes mais concentrada próxima ao valor verdadeiro do parâmetro.

Nos casos do Grupo II, onde os dados das amostras "prévia" e "atual" foram gerados com diferentes variâncias residuais, as estimativas poderiam ser obtidas de maneira aproximada pelas fórmulas (A-6) desenvolvidas no Apêndice II. Entretanto, os resultados apresentados referem-se à aplicação das fórmulas (3.42) anteriores, e as Tabelas 4 e 5 permitem verificar que as estimativas de Bayes são relativamente robustas. Contrariando a hipótese de igualdade de variâncias (sob a qual foram desenvolvidas), as estimativas de Bayes apresentaram distribuição de frequência mais concentrada em torno do 
valor verdadeiro do parâmetro que as estimativas de Mínimos Quadrados, notadamente nos 79,89 e 99 casos em que se considerou um aumento de $\sigma_{1}^{2}=36$ (amostra "prévia") para $\sigma^{2}=144$ (amostra "atua1") na variância residual. Mantida a hipótese de que os valores verdadeiros dos parâmetros sejam os mesmos, as estimativas de Bayes incorporaram, neste caso, informações "a priori" mais fidedignas (por se basearem em amostra de tamanho maior e de menor variância) que aquelas fornecidas pela amostra "atual". Para os 10 e 11 o casos,di minuindo-se o valor da variância dos dados "atuais" em relação a dos dados da amostra "prévia", a vantagem da estimativa de Bayes se verifica, de certa forma, apenas quando obtida com base em amostra an terior de tamanho bem maior que a da "atual".

\subsection{2 - Estimativas das variāncias das estimativas dos pa- rāmetros}

Os valores médios para as estimativas das variâncias das estimativas dos parâmetros, apresentados na coluna (4) da Tabela 2 tenderam aos valores verdadeiros destas variâncias e se confir maram pelos valores dos erros quadráticos médios - coluna (5) - Pa ra todos os casos do Grupo I.

As expressões que determinaram estas estimativas foram as usuais obtidas pelo método dos Mínimos Quadrados na análise dos dados da amostra "atual" e pelas fórmulas (3.42) para a análise de Bayes: 


$$
\hat{V}(a)=\frac{s^{2}}{n} \quad ; \quad \bar{V}(b)=\frac{s^{2}}{s_{x x}}
$$

e

$$
\hat{\mathrm{v}}\left(\mathrm{a}_{2}\right)=\frac{\mathrm{s}_{2}^{2}}{\mathrm{n}_{1}+\mathrm{n}} ; \quad \hat{\mathrm{V}}\left(\mathrm{b}_{2}\right)=\frac{\mathrm{s}_{2}^{2}}{\mathrm{~s}_{\mathrm{xx}}^{\prime}+\mathrm{s}_{\mathrm{xx}}}
$$

respectivamente, e, de (3.41):

$$
s_{2}^{2}=\frac{1}{\left(n_{1}+n-2\right)}\left[\begin{array}{r}
\left(n_{1}-2\right) s_{1}^{2}+(n-2) s^{2}+n_{1} a_{1}^{2}+n a^{2}+s_{x x_{1}^{\prime}}^{\prime} b_{1}^{2}+ \\
+s_{x x} b^{2}-\left(n_{1}+n\right) a_{2}^{2}-\left(s_{x x}^{\prime}+s_{x x}\right) b_{2}^{2}
\end{array}\right] .
$$

Estas expressões mostram uma qualidade vantajosa dos estimadores de Bayes em relação aos de Mínimos Quadrados, que éo de apresentarem menor variância.

Para os casos do Grupo II, os valores aproximados das estimativas destas variâncias podem ser determinados pelas expressões (A-5) encontradas no Apêndice II. Entretanto, para este estudo, os cálculos foram feitos na hipótese de as variâncias serem iguais e resultaram em valores consideravelmente menores quando obtidos pelo método de Bayes nos 79, 89 e 99 casos, onde as informações prévias são baseadas em dados de menor variância, encontrados em amostras de tamanho maior. Para estes casos, se o interesse è ter conhecimento mais exato sobre o verdadeiro valor do parâmetro, o mé todo de Bayes ofereceu estimativas de menor variância, mesmo que a situação real contrarie a hipótese de igualdade das variâncias e mes mo que a estimativa substime a verdadeira variância atual. No 10 ? caso, as estimativas de Bayes para as variâncias das estimativas dos 
parâmetros não apresentaram diferença significativa em relação àque las da análise "atual", enquanto que são maiores no 11 ọ caso, para a estimativa da variância da estimativa de $\alpha$.

\subsection{3 - Intervalos de confiança para os parāmetros}

As Tabelas 2 e 3 mostram que os valores médios das am plitudes dos Intervalos de Confiança para os parâmetros nas 200 repetições, foram menores quando foi utilizado o método bayesiano.

Além de apresentarem estimativas das variâncias das estimativas dos parâmetros menores, a anālise bayesiana opera com måior número de graus de liberdade, o que determina menor valor de "t", utilizado na determinação dos limites do intervalo de confiança.

\subsection{4 - Resultados gerais sobre a anālise de regressão si-} mulada

Os resultados discutidos nos três ültimos îtens, per mitem fazer as seguintes observações gerais:

a) Quando a variância não sofre modificação do momento em que se toma o conjunto de estatisticas suficientes para representar as informações "a priori", atē o momento em que se toma a amostra para a anālise atual, a eficiência do método bayesiano é menos notória,à medida que o tamanho da amostra "atual" aumenta. Bastaria, por exem 
plo, utilizar os mesmos dados simulados, invertendo-se a situação das amostras "atual" e "prévia" (o que é possível, pois ambas foram geradas aleatoriamente). Nesta nova situação, as estimativas de Bayes, usando Distribuições Conjugadas, são as mesmas. Entretanto, as estị mativas de Mínimos Quadrados são obtidas em amostra "atual" mais in formativa $(n=21)$ que a "prēvia" $\left(n_{1}=7 ; n_{1}=11\right.$ ou $\left.n_{1}=15\right)$.

b) De um modo geral, a violação da hipótese de que a variância é a mesma, no caso em estudo mostrou-se que os resultados, quanto à eficiência dos estimadores de Bayes, não são significativamente modificados.

c) As estimativas das variâncias das estimativas dos parâmetros sendo menores e o número de graus de liberdade maiores no método de Bayes, fizeram com que conclusõeś semelhantes fossem estendidas para o intervalo de previsão para $Y_{h}$ e intervalo de confiança para $E\left(Y_{h}\right)$, o que se pode verificar pela Figura 7 do exemplo em 4.3.

d) Os intervalos de confiança para os parâmetros, determinados neste estudo, ao nível de confiança de 95\%, for am sempre menores quando obtidos pelo método de Bayes e, nas 200 repetições de cada caso, cobriram os verdadeiros valores dos parâmetros, nas percentagens que constam da Tabela 8 . 
Tabela 8 - Percentagens dos Intervalos de Confiança de Bayes que co brem os verdadeiros valores dos parâmetros, observadas nas 200 repetições de cada caso.

\begin{tabular}{cccc}
\hline & & \multicolumn{2}{c}{ PARÂMETROS } \\
\cline { 3 - 4 } GRUPO & & $\alpha$ & $\beta$ \\
\hline & 19 & $93,5 \%$ & $95,0 \%$ \\
I & 39 & $89,0 \%$ & $97,0 \%$ \\
& 49 & $94,5 \%$ & $96,0 \%$ \\
& 59 & $93,5 \%$ & $96,5 \%$ \\
& 69 & $96,5 \%$ & $95,0 \%$ \\
& 79 & $93,0 \%$ & $97,0 \%$ \\
& 89 & $95,5 \%$ & $94,0 \%$ \\
& 99 & $95,0 \%$ & $93,0 \%$ \\
& 109 & $94,0 \%$ & $95,0 \%$ \\
& 119 & $94,0 \%$ & $97,5 \%$ \\
& & $92,5 \%$ & $98,0 \%$ \\
\hline
\end{tabular}

\section{5 - Sugestões Para Outros Estudos}

0 manuseio dos dados simulados permitiu sugerir estu dos de outras situações que não foram objetos deste trabalho:

a) Verificar, sob a hipótese de que os parâmetros $\alpha$ e $\beta$ do modelo de regressão linear simples sejam iguais nos momentos em que se tomam as amostras "prévia" e "atua1", atē que proporção as variân- 
cias podem ser modificadas, de modo a garantir a robustez do método de Bayes. Comparar estes resultados com aqueles que se podem obter de maneira aproximada quando se estudam as regressões com variâncias diferentes (Apêndice II), e verificar a precisão da aproximação deste método.

b) As Distribuições Conjugadas, usadas pelo método bayesiano neste trabalho, permitiram encontrar f.d.p. "a posteriori" de fácil manejo algébrico e com possibilidades de análise estatística semeThante àquela usada pelo método dos Mỉnimos Quadrados aplicada aos dados da amostra "atual". Nesta situação, admitir que nosso grau de confiança "a priori" sobre o parâmetro $\alpha$, por exemplo, se traduz por uma distribuição normal de média $a_{1}$ e variância $\frac{s_{1}^{2}}{n_{1}}$, significa que estamos dando um peso maior ao valor central desta distribuição como informação prévia sobre o verdadeiro valor do parâmetro. Entre tanto, pode acontecer que as informações disponíveis antes de serea lizar o experimento atual não sejam convincentes, a ponto de se admi tir maior grau de confiança a um valor particular do parâmetro. Mas o intervalo de confiança a um certo nível, obtido pelos métodos usuais na análise prévia, pode nos fornecer um intervalo de possíveis valores para o verdadeiro parâmetro. Assim, poder-se-ia deduzir as f.d.p. "a posteriori" pelo método de Bayes, baseando-se em distribuição "a priori" uniforme naquele intervalo. Esta situação não foi encontrada na literatura, a ponto de sugerir aplicação. 
117 .

5. CONCLUSÕES

A anālise do modelo de regressão linear, simples desenvolvida neste trabalho utilizou o método de Bayes para incorporar as informações sobre os parâmetros obtidos em uṇa amostra "prévia", àquelas encontradas na amostra "atua1".

Adotou-se a função perda quàdrática, para a qual o estimador de Bayes é a média da distribuição "a posteriori".

As estimativas de Bayes foram comparadas com as esti mativas de minimos quadrados, obtidas com os dados da amostra "atual", e as conclusões, de maneira geral, foram as seguintes:

a) 0 método bayesiano permite utilizar a função perda para ade quar a estimação de parâmetros, de maneira realista, ao processo de decisão, mas a escolha correta desta função para traduzir uma situa ção prätica, bem como as dificuldades algébricas nas deduções analî 
118.

ticas, têm sido limitações à aplicação do método.

b) Utilizando-se da distribuição "a priori" indicando "ignorân cia" sobre os parâmetros, as conclusões do método bayesiano são semelhantes àquelas obtidas pelo método de Mínimos Quadrados, pois to da informação sobre os parâmetros provēm apenas da amostra "atual".

c) Na situação do ítem anterior, uma vantagem do método de Bayes é que na própria expressão analítica da função de densidade de probabilidade (f.d.p.) da distribuição "a posteriori" encontramos o tipo de teste de hipótese a ser aplicado à estimativa do parâa metro.

d) Quando a variância residual é conhecida, a estimativa de Bayes para um parâmetro é a média aritmética ponderada das estimati vas "a priori" e amostral, cujos pesos são os inversos das respecti vas variâncias. A precisão (inverso da variância) da estimativa de Bayes é a soma das precisões das estimativas "a priori" e "atual".

e) Quando a variância residual é desconhecida, e as informações disponiveis sobre os parâmetros pela amostra "prévia" são expressas por estatíticas suficientes, podemos incorporä-las às informações obtidas na amostra"atual" pelo teorema de Bayes, utilizando Distribuições Conjugadas.

f) Sob a hipótese de que os dados da amostra "prévia" e da amos tra "atual" provêm da mesma população (mesma variância residual e mesmos parâmetros), o uso das Distribuições Conjugadas fornece est $\underline{i}$ 
mativas imparciais, com menor variância que aquelas obtidas pelo método de Minimos Quadrados, principalmente quando se dispõem de pequenas amostras "atuais".

g) $\mathrm{Na}$ situação do item anterior, os intervalos de confiança pa ra os parâmetros e o intervalo de previsão são menores quando se usam os estimadores de Bayes.

h) A simulação de dados, além de confirmar as conclusões dos Itens $f$ e $g$, mostrou que a estimativa de Bayes tende mais rapidamen te ao verdadeiro valor do parâmetro ao se fazerem repetições dos ex perimentos. Utilizando os mesmos estimadores de Bayes, o método se revelou robusto para pequenas diferenças entre as variâncias residuais das amostras "prévia" e "atual". 
120 .

6. LITERATURA CITADA

AITCHISON, J., 1964. Bayesian Tolerance Regions. J. Roy. Statist. Soc. B. Londres, 26: 161-175.

ANTLEMAN, G.R., 1965. Insensitivity to Non-Optimal Design in Bayesian Decision Theory. J.Am. Statist. Assoc. Washington, 60: 584-601.

AKAIKE, H., 1978. A New Look at the Bayes Procedure. Biometrika. Londres, 65: 53-59.

BARNARD, G.A., 1954. Simplified Decision Functions . Biometrika. Londres, 41: 241-251.

BARNARD, G.A., 1958. Thomas Bayes - A Bibliographical Note. Biometrika. Londres, 45: 293-295.

BARTHOLOMEW, D.J., 1965. A Comparison of Some Bayesian and Frequentist Inferences. Biometrika. Londres, 52: 19-36. 
BAYES, T., 1958. An Essay Toward Solving a Problem in the Doctrine of Chances. Biometrika. Londres, 45: 296-315.

BICKEL, P.J. e D. BLACKWELL, 1967. A Note on Bayes Estimates. Ann. Math. Statist. Baltimore, 38: 1907-1911.

BORCH, K., 1967. The Theory of Risk. J. Roy. Statist. Soc. B. Londres, 29: 432-467.

BOX, G.E.P. e G.C. TIAO, 1962. A Further, Look at Robustness Via Bayes's Theorem. Biometrika. Londres, 49: 419-432.

EFRON, B. e C. MORRIS, 1971. Limiting the Risk of Bayes and Empirica1 Bayes Estimators - Part I: The Bayes Case. J. Am. Statist. Assoc. Washington, 66: 807-815.

EVANS, I.G., 1964. Bayesian Estimation of the Variance of Normal Distribution. J. Roy. Statist. Soc. B. Londres, 26: 63-68.

EL-SAYYAD, G.M., 1967. Estimation of the Parameter of an Exponential Distribution.' J. Roy. Statist. Soc. B. Londres, 29: 525-532 .

FERGUSON, T.S., 1969. Mathematical Statistics. 3. Edição. Nova York, Academic Press, 396 p.

FREEMAN, H., 1963. Introduction to Statistical Inference. Massashusetts, Addison-Wesley. 445 p.

HADLEY, G., 1967. Introduction to Probability and Statistical Decision Theory. São Francisco, Holden-Day. 570 p. 
HARTIGAN, J., 1964. Invariant Prior Distributions. Ann. Math. Statist. Baltimore, 35: 836-845.

HARTIGAN, J., 1965. The Assymptotically Umbiased Prior Distributions. Ann. Math. Statist. Baltimore, 36: 1137-1152.

HARTIGAN, J., 1969. Linear Bayesian Methods. J. Roy. Statist. Soc. B. Londres, 31: 446-454.

HOFFMANN, R., 1980. Estatística para Economistas. Sã• Paulo, Livraria Pioneira Editora. 379 p.

HOFFMANN, R. e S. VIEIRA, 1977. Anālise de Regressão - Uma Introdução à Econometria. São Paulo, HUCITEC-EDUSP. 339 p.

HOOG, R.V. e A.T. CRAIG, 1959. Introduction to Mathematical Statiscs. Nova York, Macmillan. 438 p.

JEFFREYS, H., 1948. Theory of Probability. 2. Edição. Oxford, Clarendon Press. 578 p.

LEHMANN, E.L., 1950. Some Principles of the Theory of Testing..Hypotheses. Ann. Math. Statist. Baltimore, 21: 1-26.

LINDLEY, D.V., 1953: Statistical Inference. J. Roy. Statist. Soc. B. Londres, 15: 30-76.

LINDLEY, D.V., 1956. On a Measure of the Information Provinded by an Experiment. Ann. Math. Statist. Baltimore, 27: 986-1005.

LINDLEY, D.V., 1957. Binomial Sampling Schemes and the Concept of Information. Biometrika. Londres, 44: 179-186. 
LINDLEY, D.V., 1965. Introduction to Probability and Statistics. from a Bayesian Viewpoint. Volume II. Cambridge, University Press. 291 p.

LINDLEY, D.V., 1972. Bayesian Statistics - A Review. Philadelphia, Society for Industrial and Applied Mathematics. 83 p.

LINDLEY, D.V. e G.M. EL-SAYYAD, 1968. The Bayesian Estimation of Li near Functional Relationships. J. Roy. Statist. Soc. B. Londres, 30: $190-202$.

LINDLEY, D.V. e A.F.M. SMITH, 1972. Bayes Estimates for the Linear Model. J. Roy. Statist. Soc. B. Londres, 34: 1-41.

McMILLAN, B., 1953. The Basic Theorems of Information Theory. Ann. Math. Statist. Baltimore, 24: 196-219.

MOOD, A.M. e F.A. GRAYBILL, 1972. Introducción a la Teoría de la Estadística. $4{ }^{\mathrm{a}}$. Edição. Madrid, Aguilar Ediciones. 536 p. Tra dução de Rafael Pro Bermejo.

PLACKETT, R.L., 1966. Current Trends in Statistical Inference. J. Roy. Statist. Soc. A. Londres, 129: 249-267.

PRATT, J.W., 1964. Risk Aversion in the Small and in the Large. Econometrica. Clevedon, 32: 122-136.

PRATT, J.W., 1965. Bayesian Interpretation of Standard Inference Stataments. J. Roy. Statist. Soc. B. Londres, 27: 169-203.

PRATT, J.W.; H. RAIFFA e R. SCHALAIFER, 1964. The Foundation of Decision Under Uncertainty: an Elementary Exposition. J. Am. Sta-. tist. Assoc. Washington, 59: 353-375. 
PUGACHEV, V.S., 1973. Introducción a la Teoria de las Probabilidades. Moscou, Editorial Mir. 323 p. Tradução de A. Samojválov.

RAIFFA, H., 1977. Teoria da Decisão. São Paulo, Vozes-EDUSP. 346 p. Tradução de Sérgio Ellery Girão Barroso.

RAIFFA, H. e R. SCHALAIFER, 1961. Applied Statistical Decision Theory. Boston, Harvard University. 355 p.

ROTHENBERG, T.J., 1973. Efficient Estimation with a Priori Information. Londres, Yale University Press. 181 p.

ROTHEMBERG, T.J., 1974. The Bayesian Approach and Alternatives in Econometrics - II. In: FIENBERG, S.E. e A. ZELLNER. Studies in Bayesian Econometrics and Statistics. North-Holand. p. 55-67.

SAVAGE, L.J., 1954. The Foundations of Statistics. Nova York, John Wiley. 294 p.

SILVEY, S.D., 1978. Statistical Inference. 3. Edição. Londres, Chap man and Hall. 191 p.

SMITH, A.F.M., 1973. A General Bayesian Linear Model. J. Roy. Statistic. Soc. B. Londres, 35: 67-75.

THATCHER, A.R., 1964. Relationships Between Bayesian and Confidence Limits for Predictions. J. Roy. Statist. Soc. B. Londres, 26: 176-192.

THEIL, H., 1963. On the Use of Incomplet Prior Information in Regression Analysis. J. Am. Statist. Assoc. Washington, 58: 401-414 . 
TIAO, G.C. e G.E.P. BOX, 1974. Some Comments on "Bayes" Estimator. In: FIENBERG, S.E. e A. ZELLNER. Studies in Bayesian Econometrics. and Statistics. North-Holland. p. 619-626.

TIAO, G.C. e A. ZELLNER, 1964. Bayes's Theorem and the Use of Prior Knowledge in Regression Analysis. Biometrika. Londres, 51: 219-230 .

VARIAN, H.R., 1974. A Bayesian Approach to Real Estate Assessment. In: FIENBERG, S.E. e A. ZELLNER. Studies in Bayesian Econometrics. and Statistics. North-Holland. p. 195-208.

WALD, A., 1939. Contributions to the Theory of Statistical Estimation and Testing Hypotheses. Ann. Math. Statist. Baltimore, 10: 299-326.

WALD, A., 1950. Statistical Decision Functions. Nova York, John Wi1ey. 179 p.

WINKLER, R.L., 1967. The Assessment of Prior Distributions in Bayesian Analysis. J. Am. Statist..Assoc. Washington, 62: 776-800.

WONNACOTT, R.J. e T.H. WONNACOTT, 1976. Econometria. Rio de Janei rio, Livros Técnicos e Científicos Editora Ltda. 424 p. Tradução de Maria C. Silva.

ZACKS, S., 1971. The Theory of Statistical Inference. Nova York, John Wiley. 609 p.

ZELLNER, A., 1971. An Introduction to Bayesian Inference in Econometrics. Nova York, John Wiley. 423 p. 
ZELLNER, A., 1974. The Bayesian Approach and Alternatives in Econo metrics - I. In: FIENBERG, S.E. e A. ZELLNER. Studies in Bayesian Econometrics and Statistics. North-Holland. p. 39-5.4.

ZELLNER, A. e V.K. CHETTY, 1965. Prediction and Decision Problems in Regression Models From the Bayesian Point of View. J. Am. Statist. Assoc._Washington, 60: 608-616. 
127.

\section{APENDICE I \\ PROGRAMA UTILIZADO NA SIMULAÇÃO E 'ANÁL ISE DOS DADOS}


128.

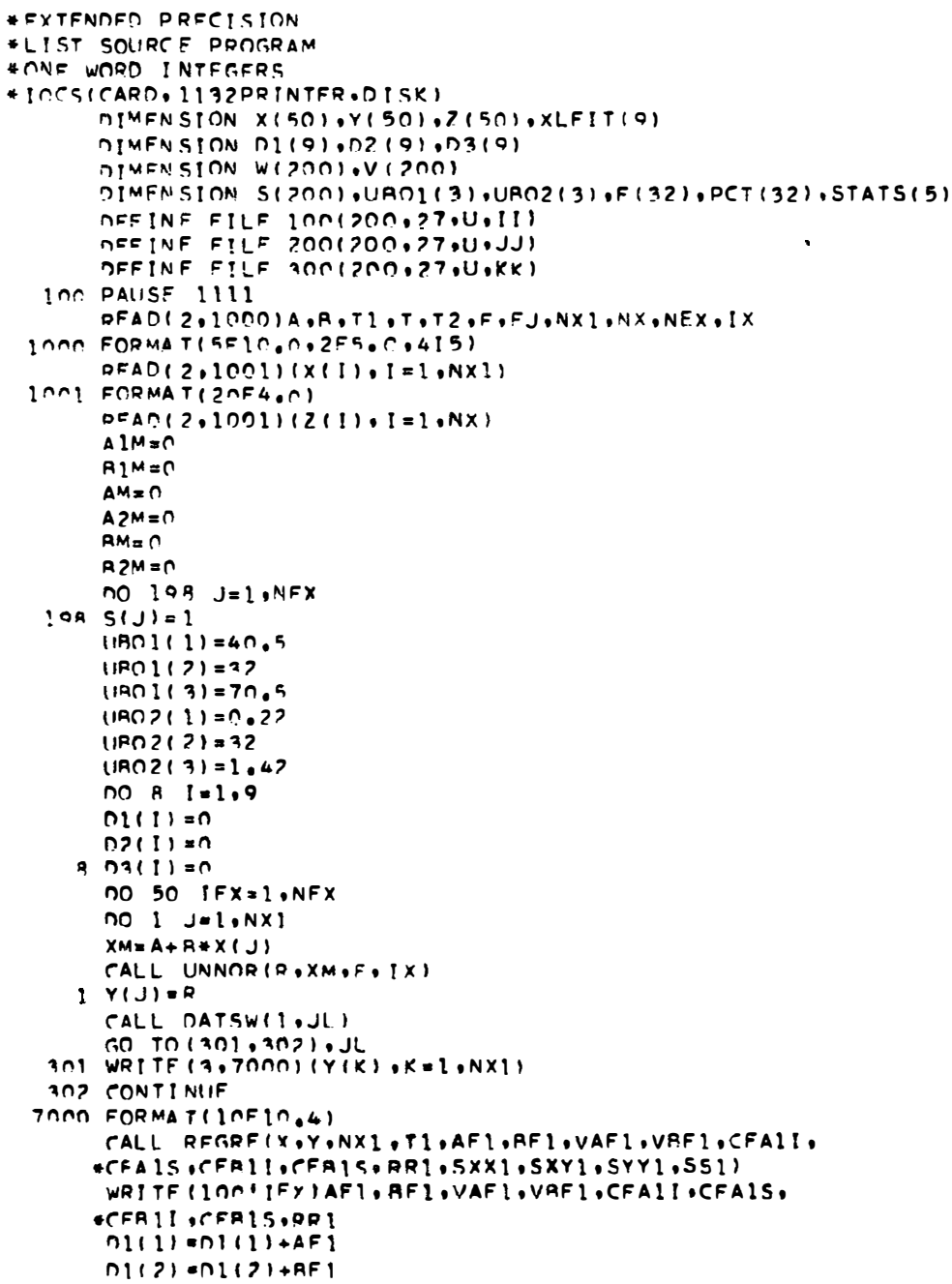


129.

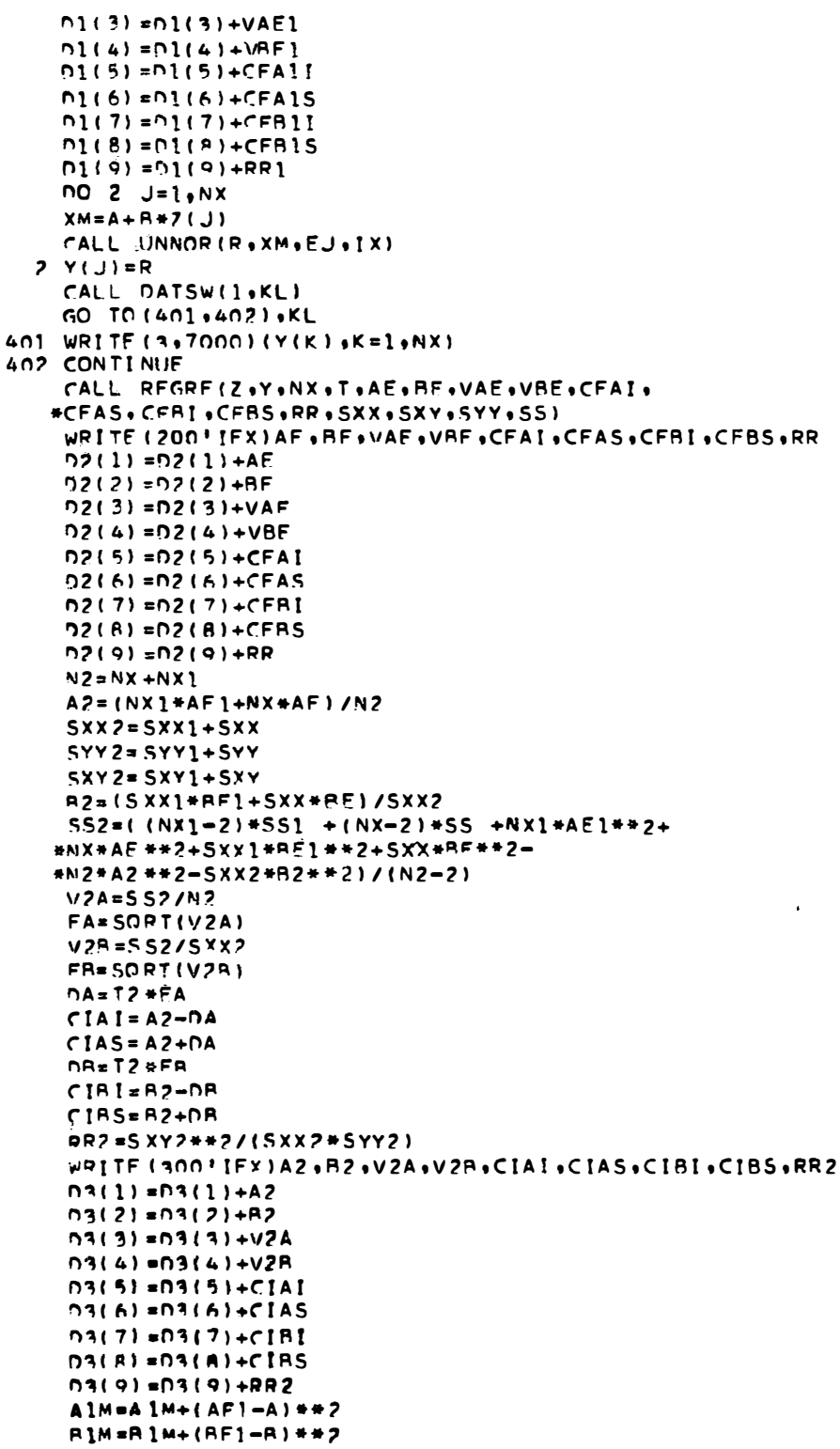




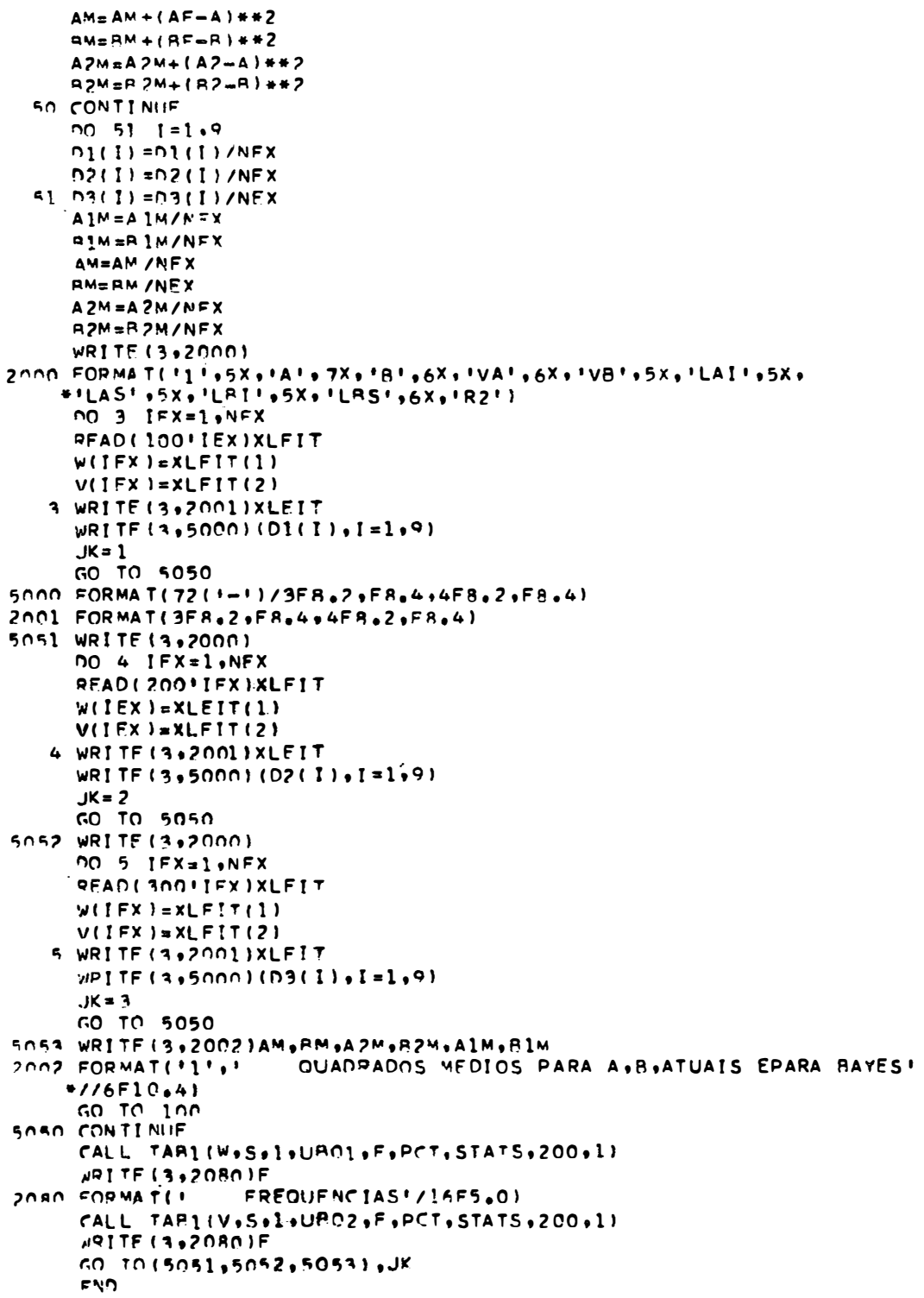


131.

\section{APENDICE II \\ REGRESSÕES COM VARIÂNCIAS DIFERENTES}


132.

\section{REGRESSÕES COM VARIÂNCIAS DIFERENTES}

Em algumas situações, temos motivos para considerar que no período entre a realização do experimento que nos forneceu as estatỉsticas para informações "a priori" e a realização do experimento "atual", tenha ocorrido modificação no valor da variância residual, embora os parâmetros sejam os mesmos. Nestas condições, a de dução dos estimadores dos parâmetros e variâncias, se faz da maneira a seguir, com base no artigo de TIAO e ZELLNER (1964).

Consideremos os modelos de regressão "a priori" e "atual", respectivamente:

$$
\begin{array}{ll}
Y_{i}=\alpha+\beta x_{i}+e_{i} & \left(i=1,2, \ldots, n_{1}\right) \\
Y_{j}=\alpha+\beta x_{j}+e_{j} & (j=1,2, \ldots, n)
\end{array}
$$

onde $e_{i} \cap N\left(0 ; \sigma_{1}^{2}\right)$ e $e_{j} \cap N\left(0 ; \sigma^{2}\right)$. 
Por (3.11) podemos escrever para a f.d.p. "a priori":

$$
\begin{gathered}
\pi_{1}\left(\alpha ; \beta ; \sigma_{1}^{2}\right) \propto\left(\sigma_{1}^{2}\right)^{-1 / 2\left(n_{1}+2\right)} \cdot \exp \left\{-\frac{1}{2 \sigma_{1}^{2}}\left[v_{1} s_{1}^{2}+n_{1}\left(\alpha-a_{1}\right)^{2}+S_{x x}^{1}(\beta-b)^{2}\right]\right\} \\
E, \operatorname{para} \text { a amostra "atual": } \\
p\left(y \mid \alpha ; \beta ; \sigma^{2}\right) \propto\left(\sigma^{2}\right)^{-1 / 2(n+2)} \cdot \exp \left\{-\frac{1}{2 \sigma^{2}}\left[v s^{2}+n(\alpha-a)^{2}+s_{x x}(\beta-b)^{2}\right]\right\}
\end{gathered}
$$$$
\text { A f.d.p. "a posteriori" será: }
$$

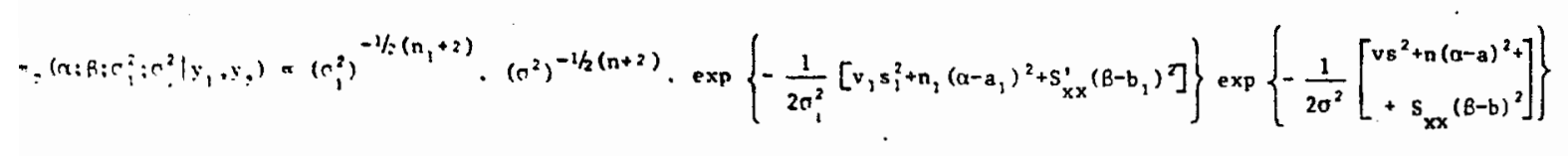$$
\text { Integrando em relação a } \sigma_{1}^{2} \text { e } \sigma^{2} \text { teremos: }
$$$$
\pi_{2}\left(\alpha ; \beta \mid y_{1}, y, \sigma_{1}^{2}, \sigma^{2}\right) \propto \int_{0}^{\infty}\left(\sigma_{1}^{2}\right)^{-1 / 2\left(n_{1}+2\right)} \cdot e^{-\left(k_{1} / 2 \sigma_{1}^{2}\right)}\left[\begin{array}{cc}
\int_{0}^{\infty}\left(\sigma^{2}\right)^{-1 / 2(n+2)} \times x \\
x & e^{-\left(k / 2 \sigma^{2}\right)} d \sigma^{2}
\end{array}\right] d \sigma_{1}^{2}
$$$$
\pi_{2}\left(\alpha ; \beta \mid y_{1}, y, \sigma_{1}^{2} ; \sigma^{2}\right) \propto k_{1}^{-\left(n_{1} / 2\right)} \cdot k^{-\pi / 2}
$$$$
\text { onde } k=\left[v s^{2}+n(\alpha-a)^{2}+s_{x x}(\beta-b)^{2}\right] \text {. }
$$

Fazendo:

$$
\begin{array}{ccc}
Q=\frac{n(\alpha-a)^{2}+s_{x x}(\beta-b)^{2}}{s^{2}} & \text { e } & Q_{1}=\frac{n_{1}\left(\alpha-a_{1}\right)^{2}+S_{x x}^{\prime}\left(\beta-b_{1}\right)^{2}}{s_{1}^{2}} \\
v=n-2 & \text { e } & v_{1}=n_{1}-2
\end{array}
$$




$$
\mathrm{s}^{2}=\mathrm{vs}^{2} \quad \mathrm{e} \quad \mathrm{s}_{1}^{2}=\mathrm{v}_{1} \mathrm{~s}_{1}^{2},
$$

teremos :

$$
\begin{aligned}
& \pi_{2}\left(\alpha ; \beta \mid y_{1}, y_{2}\right) \propto\left[1+\frac{Q_{1}}{v_{1}}\right]^{-\left(n_{1} / 2\right)} \cdot\left[1+\frac{Q}{v}\right]^{-n / 2} \\
& \pi_{2}\left(\alpha ; \beta \mid y_{1}, y_{2}\right) \propto\left[1+\frac{Q_{1}}{v_{1}}\right]^{-1 / 2\left(v_{1}+2\right)} \cdot\left[1+\frac{Q}{v}\right]^{-1 / 2(v+2)}
\end{aligned}
$$

Desenvolvendo cada potência:

$$
\left[1+\frac{Q}{v}\right]^{-1 / 2(v+2)}=e^{-1 / 2(v+2) L[1+(Q / v)]}
$$

mas, pela fórmula de Taylor:

$$
\begin{gathered}
\mathrm{L}\left(1+\frac{\mathrm{Q}}{\mathrm{v}}\right)=\frac{\mathrm{Q}}{\mathrm{v}}-\frac{\mathrm{I}}{2}\left(\frac{\mathrm{Q}}{\mathrm{v}}\right)^{2}+\frac{\mathrm{l}}{3}\left(\frac{\mathrm{Q}}{\mathrm{v}}\right)^{3}-\ldots=\frac{\mathrm{Q}}{\mathrm{v}}+\mathrm{R} \\
{\left[1+\frac{\mathrm{Q}}{\mathrm{v}}\right]^{-1 / 2(\mathrm{v}+2)}} \\
=\mathrm{e}^{-1 / 2(\mathrm{v}+2)[(\mathrm{Q} / \mathrm{v})+\mathrm{R}]} \\
=\mathrm{e}^{-\mathrm{Q} / 2} \cdot \mathrm{e}^{-1 / 2\{[2(\mathrm{Q} / \mathrm{v})]+(\mathrm{v}+2) \mathrm{R}\}}
\end{gathered}
$$

Adaptando o desenvolvimento pela fórmula de Mac-Laurin feito por TIAO e ZELLNER, encontra-se:

$$
e^{-1 / 2\{[2(Q / v)]+(v+2) R\}}=\sum_{j=0}^{\infty} q_{j} v^{-j}
$$


onde: $\mathrm{q}_{0}=1$

$$
\begin{aligned}
& q_{1}=\frac{1}{4}\left[Q^{2}-4 Q\right] \\
& q_{2}=\frac{1}{96}\left[3 Q^{4}-40 Q^{3}+192 Q^{2}\right] \\
& \ldots \ldots \\
& \text { Logo, (A.1) fica : } \\
& \pi_{2}\left(\alpha ; \beta \mid y_{1} ; y\right) \propto e^{-1 / 2\left(Q_{1}+Q\right)} \cdot \sum_{i=0}^{\infty} p_{i} v_{i}^{-i} \sum_{j=0}^{\infty} q_{j} v^{-j}
\end{aligned}
$$

Assim, (A.2) representa o desenvolvimento assintótico da f.d.p. "t"-dupla de (A.1) e, para analisarmos, consideremos apenas o termo principal do desenvolvimento:

$$
\pi_{2}\left(\alpha ; \beta \mid y_{1} ; y\right) \dot{\alpha} e^{-1 / 2\left(Q_{1}+Q\right)},
$$

onde $\propto$ indica "aproximadamente proporcional a".

Usando, por facilidade algébrica, a forma matriciá,

podemos escrever:

$$
\begin{aligned}
Q_{1}+Q & =\frac{1}{s_{1}^{2}}\left[n_{1}\left(\alpha-a_{1}\right)^{2}+s_{x x}^{\prime}\left(\beta-b_{1}\right)^{2}\right]+\frac{1}{s^{2}}\left[n(\alpha-a)^{2}+s_{x x}(\beta-b)^{2}\right] \\
& =(B-\bar{B})^{\prime} \cdot \text { D. }(B-\bar{B})
\end{aligned}
$$

onde:

$$
\begin{aligned}
\bar{B}=D^{-1}\left(M_{1} \cdot \widehat{B}_{1}+M \cdot \widehat{B}\right) & \bar{e} \text { o vetor média do termo principal do. } \\
& \text { desenvolvimento assintótico, e: }
\end{aligned}
$$




$$
\begin{aligned}
& \mathrm{M}_{1}=\frac{1}{\mathrm{~s}_{1}^{2}} \mathrm{X}_{1}^{\mathrm{P}} \mathrm{X}_{1} ; \quad M=\frac{1}{\mathrm{~s}^{2}} \mathrm{X}^{\prime} \mathrm{X} \\
& \widehat{\mathrm{B}}_{1}=\left(\mathrm{X}_{1}^{\prime} \mathrm{X}_{1}\right)^{-1} \mathrm{X}_{1}^{\prime} \mathrm{y}_{1} ; \quad \widehat{B}=\left(\mathrm{X}^{\prime} \mathrm{X}\right)^{-1} \mathrm{X}^{\prime} \mathrm{Y} \\
& \mathrm{D}=\mathrm{M}_{1}+\mathrm{M}=\frac{1}{\mathrm{~s}_{1}^{2}} \mathrm{X}_{1}^{\prime} \mathrm{X}_{1}+\frac{1}{\mathrm{~s}^{2}} \mathrm{X}^{\prime} \mathrm{X}
\end{aligned}
$$

Assim teremos:

$$
\mathrm{D}=\frac{1}{\mathrm{~s}_{1}^{2}}\left[\begin{array}{cc}
\mathrm{n}_{1} & 0 \\
0 & \mathrm{~s}_{\mathrm{xx}}^{\prime}
\end{array}\right]+\frac{1}{\mathrm{~s}^{2}}\left[\begin{array}{cc}
\mathrm{n} & 0 \\
0 & \mathrm{~s}_{\mathrm{xx}}
\end{array}\right]=\left[\begin{array}{cc}
\frac{\mathrm{n}_{1}}{\mathrm{~s}_{1}^{2}}+\frac{\mathrm{n}}{\mathrm{s}^{2}} & 0 \\
0 & \frac{\mathrm{s}_{\mathrm{xx}}^{\prime}}{\mathrm{s}_{1}^{2}}+\frac{\mathrm{s}_{\mathrm{xx}}}{\mathrm{s}^{2}}
\end{array}\right]
$$

A matriz das estimativas de variâncias e covariâncias das estimativas dos parâmetros será:

$$
\mathrm{D}^{-1}=\left[\begin{array}{cc}
\frac{1}{\mathrm{n}_{1}+\frac{\mathrm{n}}{\mathrm{s}_{1}^{2}}} & 0 \\
0 & \frac{1}{\mathrm{~s}^{2}} \\
\frac{-\frac{\mathrm{xx}}{\mathrm{s}_{1}^{2}}+\frac{\mathrm{s}_{\mathrm{xx}}}{\mathrm{s}^{2}}}{\mathrm{~s}_{1}}
\end{array}\right]
$$

Determinemos a matriz das estimativas dos parâmetros

$(\bar{B})$. De (A.4) podemos escrever:

$$
M_{1} \widehat{B}_{1}=\frac{1}{s_{1}^{2}} X_{1}^{\prime} X_{1}\left(X_{1}^{\prime} X\right)^{-1} X_{1}^{\prime} y_{1}=\frac{1}{s_{1}^{2}}\left[\begin{array}{l}
\sum_{1} Y_{i} \\
S_{x y}^{\prime}
\end{array}\right] \text { e } M \widehat{B}=\frac{1}{s^{2}}\left[\begin{array}{ll}
n_{j} \\
S_{x y}
\end{array}\right]
$$


137.

Então:

$\vec{B}=D^{-1}\left(M_{1} \widehat{B}_{1}+M . \widehat{B}\right)=D^{-1} \cdot\left[\begin{array}{c}\frac{n_{1} a_{1}}{s_{1}^{2}}+\frac{n a}{s^{2}} \\ \frac{S_{x x_{1}}^{\prime} b_{1}}{s_{1}^{2}}+\frac{S_{x x} b}{s^{2}}\end{array}\right]$

$$
\bar{B}=\left[\begin{array}{c}
\frac{\frac{n_{1} a_{1}}{s_{1}^{2}}+\frac{n a}{s^{2}}}{\frac{n_{1}}{s_{1}^{2}}+\frac{n}{s^{2}}} \\
\frac{s_{x x_{1}^{\prime}}^{\prime} b_{x x}}{s_{1}^{2} \cdot \frac{s^{2}}{s_{x x}^{\prime}}+\frac{s_{x x}}{s^{2}}}
\end{array}\right]
$$

Logo, pela aproximação assintótica, os estimadores de Bayes para os parâmetros podem ser escritos:

$$
a_{2}=\frac{\frac{n_{1} a_{1}}{s_{1}^{2}}+\frac{n a}{s^{2}}}{\frac{n_{1}}{s_{1}^{2}}+\frac{n}{s^{2}}} . \quad \text { b } b_{2}=\frac{\frac{S_{x x_{1}^{\prime}}^{\prime} b_{1}}{s_{1}^{2}}+\frac{s_{x x} b}{s^{2}}}{\frac{S_{x x}^{\prime}}{s_{1}^{2}}+\frac{S_{x x}}{s^{2}}}
$$


138.

Utilizando a definição de precisão mencionada em 3.3.1, pode-se apresentar as expressões de (A.6) nas formas:
$a_{2}=\frac{a_{1} \cdot h_{a}^{\prime}+a \cdot h_{a}}{h_{a}^{\prime}+h_{a}}$
$b_{2}=\frac{b_{1} \cdot h_{b}^{\prime}+b \cdot h_{b}}{h_{b}^{\prime}+h_{b}}$
(A.7)

As estimativas das variâncias das estimativas dos pa râmetros ficariam, por (A.5):

$$
\hat{\mathrm{v}}\left(\mathrm{a}_{2}\right)=\frac{1}{\mathrm{~h}_{\mathrm{a}}^{\prime}+\mathrm{h}_{\mathrm{a}}} \quad \text { e } \quad \hat{\mathrm{v}}\left(\mathrm{b}_{2}\right)=\frac{1}{h_{b}^{\prime}+h_{b}}
$$

